\title{
SIMULTANEOUS SPATIAL AND TEMPORAL FOCUSING IN NONLINEAR MICROSCOPY
}

\author{
A Dissertation \\ Presented to the Faculty of the Graduate School \\ of Cornell University \\ In Partial Fulfillment of the Requirements for the Degree of \\ Doctor of Philosophy
}

\author{
by \\ Michael Earle Durst
}

August 2009 
(C) 2009 Michael Earle Durst 


\title{
SIMULTANEOUS SPATIAL AND TEMPORAL FOCUSING IN NONLINEAR MICROSCOPY
}

\author{
Michael Earle Durst, Ph. D.
}

Cornell University 2009

\begin{abstract}
Multiphoton microscopy (MPM) has become a powerful tool for imaging biological samples due to its ability to perform optical sectioning. MPM yields many advantages over standard one-photon imaging: a deeper penetration depth due to longer wavelength excitation, confinement of the focal volume, and reduced photodamage. These properties allow MPM to image samples non-invasively, acting as a form of optical biopsy for cancer research.

Simultaneous spatial and temporal focusing (SSTF), when combined with nonlinear microscopy, can improve the axial excitation confinement of wide-field and line-scanning imaging. Because two-photon excited fluorescence depends inversely on the pulse width of the excitation beam, SSTF decreases the background excitation of the sample outside of the focal volume by broadening the pulse width everywhere but at the geometric focus of the objective lens. Also, SSTF can scan the temporal focal plane axially by adjusting the group-delay dispersion (GDD) in the excitation beam path. We further discuss this technique for axial-scanning multiphoton fluorescence fiber probes without any moving parts at the distal end. The temporal focusing effect in SSTF essentially replaces the focusing of one spatial dimension in conventional wide-field and line-scanning imaging. Although the best axial confinement achieved by SSTF cannot surpass that of a regular point-scanning system, this trade-off between spatial and temporal focusing can provide significant
\end{abstract}


advantages in applications such as high-speed imaging and remote axial scanning in an endoscopic fiber probe.

We also present two new techniques for tunable dispersion compensation that are low cost, high speed, broadband, capable of high intensities, and have a large tuning range. By rotating a cylindrical lens at the Fourier plane of a folded 4-f grating pair system, the group-delay dispersion can be tuned over a range greater than $10^{5} \mathrm{fs}^{2}$, sufficient for compensating the dispersion of several meters of optical fiber. We also show that a single-element piezo bimorph mirror can generate GDD in a folded 4-f grating pair setup. With a kilohertz sinusoidal drive voltage applied to the piezo bimorph, we demonstrate high-speed axial scanning in an SSTF setup at a rate of 2 kilohertz over a range of 100 microns. 


\section{BIOGRAPHICAL SKETCH}

Michael Earle Durst grew up in Plant City, FL, the winter strawberry capital of the world. He claims Plant City as his hometown because it served as his home address for the longest amount of time. Michael was born in Ft. Myers, FL, went to kindergarten in Port Charlotte, FL, first through third grade in Alexandria, VA, fourth through part of sixth grade in Sarasota, FL, seventh and eighth grade in Tampa, FL, and all of high school in Plant City, FL.

Durst enrolled at Georgetown University in Washington, DC in the fall of 1999. He had expressed his interest in physics as a major when he filled out the application and never bothered to change it. He explored the possibilities of becoming a pre-med major, but he realized that being a doctor was not for him after training to become an EMT with the Georgetown Emergency Response Medical Service (GERMS). During his junior year, Michael decided to study abroad at Heriot-Watt University in Edinburgh, Scotland. There, he enrolled for two terms on the trimester schedule, in which he took many courses in electronics, solid state, and optical physics. Luckily, the curriculum in the United Kingdom is far different from that in the U.S., for there was no homework, only intensive final exams. This allowed Michael to travel all over the United Kingdom, Western Europe, and Northern Africa.

Durst returned to Georgetown for his senior year when he realized that he should maybe start worrying about his next phase in life. He signed up for the physics GRE about a week before the exam was to take place, got a horrible score, and the only school he could get into was Cornell University. After receiving his Bachelor of Science degree from Georgetown University in May of 2003, Michael moved to sunny Ithaca, NY. At the first orientation event for new graduate students at Cornell University, Michael Durst first met his wife, Alison. He had access to a cheap, clean, 
and non-violent Laundromat, and she had a car for grocery shopping. It was a match made in heaven. Eventually, this relationship evolved into a beautiful marriage in September 2008. During that time, Michael joined the research group of Chris Xu, where he has been working on optics research ever since. 
All my life I have wandered in darkness.

I look to my left. Nothing.

I look to my right. Pitch black.

I continue to look, turning in circles,

But I see nothing.

But alas! I see a light.

It is so beautiful. I just want to feel its presence.

I run towards it, but I seem to make no progress.

The faster I run, the faster it moves away.

For years now I have been following this light,

But not once has it stopped to follow me.

Not once has it bothered to acknowledge my existence.

I fear one day I will have to give up my quest

For this source of all that is beautiful.

And should this day ever come,

I just hope that you

Will never let your light burn out.

So that the rest of the world can one day

Behold your beauty and your grace.

And maybe, just maybe,

Your light will become more than just a beacon for lost souls,

But also a source of warmth for that one special someone

From whom you finally stop running.

-MED, English class, 1998 


\section{ACKNOWLEDGMENTS}

My career as a graduate student at Cornell University has been the most challenging time of my life. The first week of classes proved that I was the dumbest one here, and I immediately began to feel like a fraud. Yet somehow I passed the qualifying exam, survived my admission to candidacy exam, and submitted this dissertation. I never thought that I could make it this far, and I certainly could not have done it without being surrounded by a very supportive group of people.

I would like to thank my advisor, Prof. Chris $\mathrm{Xu}$, for taking a risk by hiring me despite my liberal arts background. He taught me through example what it takes to be a good researcher: you have to be the expert on every aspect of your research; you must never take any criticism personally but instead learn and grow form it; and you must always hold yourself to the highest standards. He demonstrates a skilled ability to compartmentalize personal, work, and home relationships in order to maximize the amount of attention dedicated to each. One can only hope to someday operate at the same intellectual level as Chris $\mathrm{Xu}$.

I would also like to thank the members of my research group. We have come a long way since it was just four graduate students in one office. Now, we are overflowing with five desks in the lab and even more to come down the hall. I credit the growth of our lab not only to the excellent research that we perform but also to the light-hearted and friendly atmosphere that makes us all happy to come to work. Some say that you do not have to get along with your coworkers, but it certainly helps if you do. From our escalating baking competitions and group basketball games to the huge tables at Sangham and the discovery of five dollar lunches at Risley, I am truly grateful for all the members of our research group.

I would like to thank the older members of the group to whom I am especially 
indebted. I want to thank Jim van Howe for making me feel welcome as a part of the group; for showing me the ropes; for teaching me everything I know about fibers, autocorrelations, phase modulators, and triathlons; and for showing me what it is like to live the dream of graduating with a Ph.D., having a beautiful wife and daughter, and teaching at one's alma mater. Thanks to Kristen Reichenbach for listening as I whined about work, for helping to keep the office sane with her sarcastic wit (just kidding!), and for showing me who is the best baker in the group. Jennifer Lee has served as the senior lab member over the past two years, and I would never be able to graduate if it were not for her help. I want to thank Jen for staying up late to watch Ernie and Kenny make fun of Sir Charles, for schooling me in basketball, and for showing me how Chinese food should really taste. Finally, I want to thank Demirhan Kobat for being all around awesome. I hope he can find a new punching bag when I am gone.

I would also like to thank my friends in applied physics that have put up with my abuse over the years. Thanks to Leon Bellan and Jacob Robinson for being great roommates for four years, for whipping me at video games, and for supporting me when Alison moved away. I want to thank the lunch crowd consisting of Judy Cha, Alex Kwan, and Sara Maccagnano. I want to thank my bowling team members: Mark Foster, Yoshi Okawachi, and Jay Sharping. Thanks to Chris Hensley for our softball intramural title and Dan Broaddus for being all around hilarious.

Thanks to my family for all their support. Thank you Lauren for not rubbing it in too much that you got your Master's degree before I did. Thanks Mom and Dad for always encouraging me to do my best. Finally, thank you Alison for everything. You know that I could not have made it without your support. 


\section{TABLE OF CONTENTS}

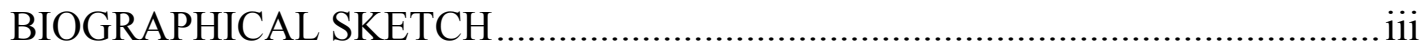

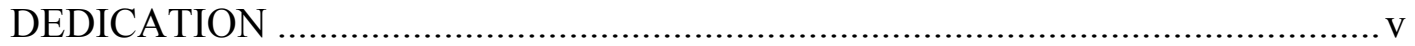

ACKNOWLEDGMENTS ......................................................................... vi

TABLE OF CONTENTS …....................................................................... viii

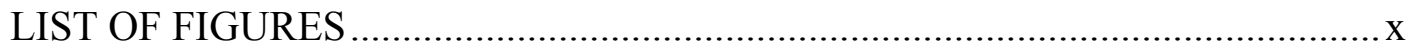

LIST OF TABLES …..............................................................................

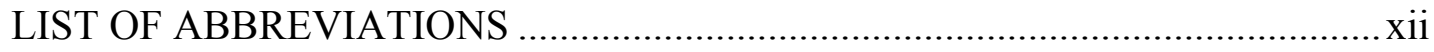

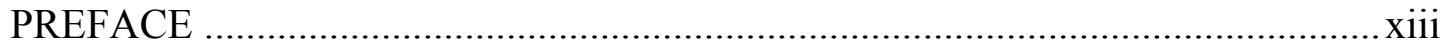

$\begin{array}{ll}\text { 1. Introduction } & 1\end{array}$

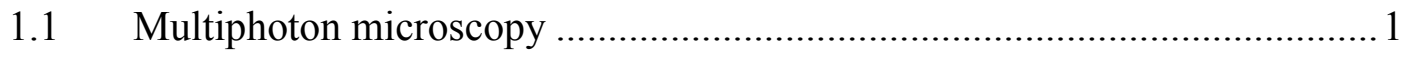

1.2 Two-photon microscopy ..................................................................... 1

1.3 Simultaneous spatial and temporal focusing ............................................. 3

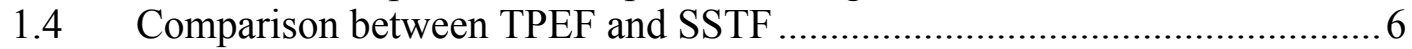

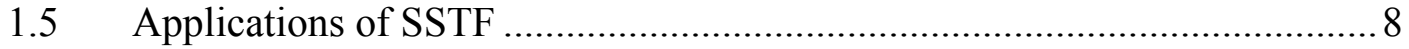

2. Simultaneous spatial and temporal focusing in nonlinear microscopy 13

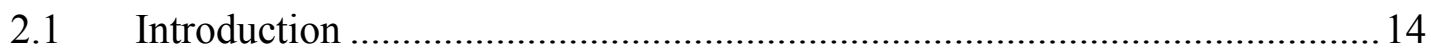

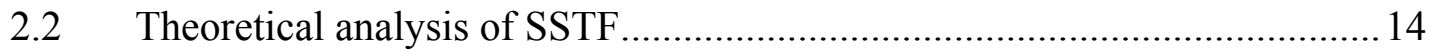

2.2.1 Temporal pulse-width evolution................................................. 14

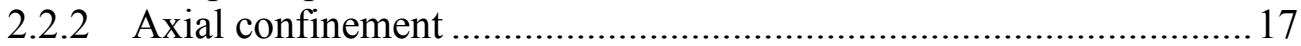

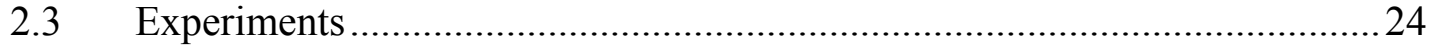

2.3.1 Experiment on temporal pulse-width evolution ............................224

2.3.2 Experiment on axial confinement..................................................2 27

2.4 Discussion on axial confinement enhancement........................................29

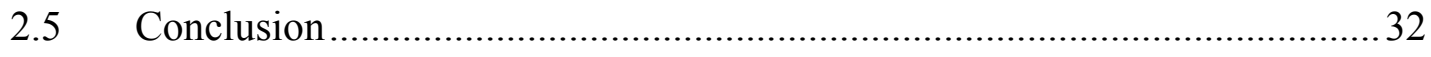

3. Simultaneous spatial and temporal focusing for axial scanning $\quad 35$

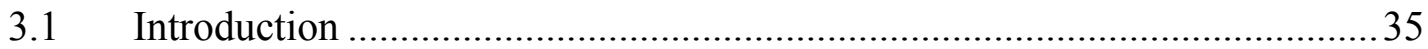

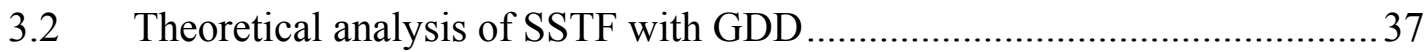

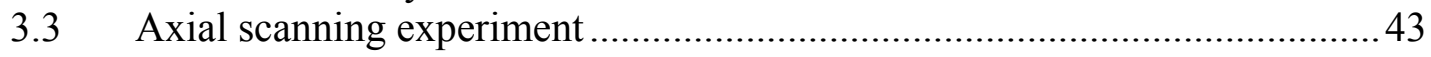

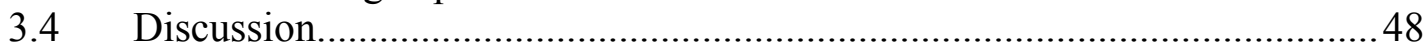

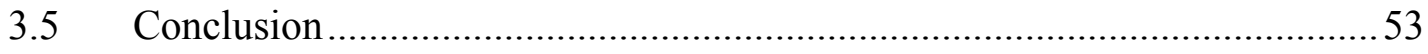

4. Tunable dispersion compensation by a rotating cylindrical lens $\quad 59$

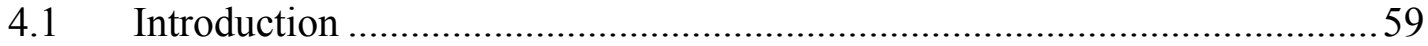

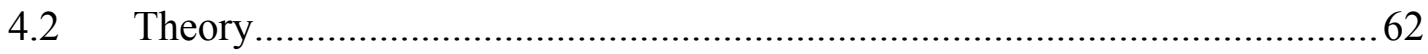

4.3 Experiments on pulse width broadening and imaging quality ....................63

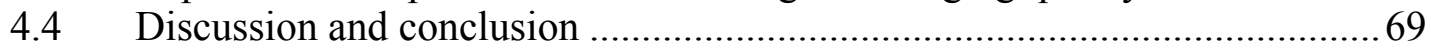


5. Kilohertz dispersion tuning in a temporal focusing setup

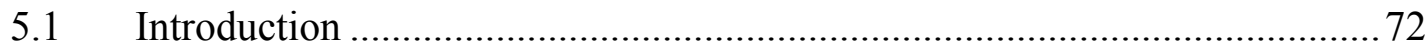

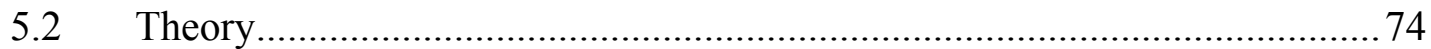

5.3 Experiments on GDD tuning and high-speed SSTF ...................................76

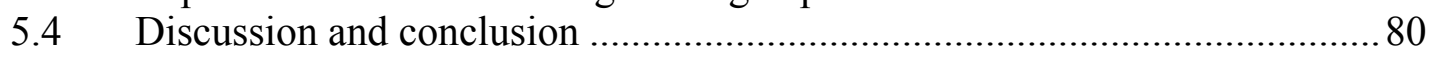

Appendices

A. Enhanced axial confinement of sum frequency generation in a temporal $\begin{array}{ll}\text { focusing setup } & 86\end{array}$

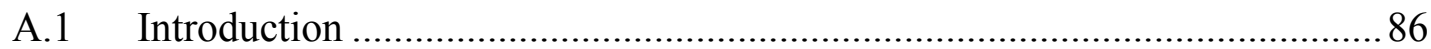

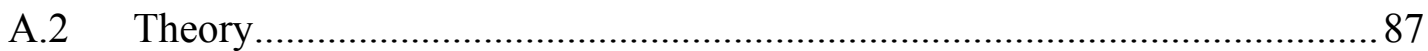

A.3 Experiment on enhanced axial confinement............................................... 89

A.4 Discussion........................................................................................ 93

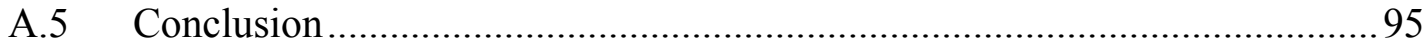

B. $\quad$ Detailed analysis of SSTF axial scanning with GDD tuning 98

C. Effects of refractive-index mismatch and scattering on simultaneous $\begin{array}{ll}\text { spatial and temporal focusing } & 101\end{array}$

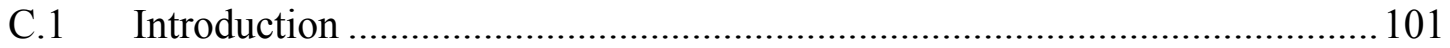

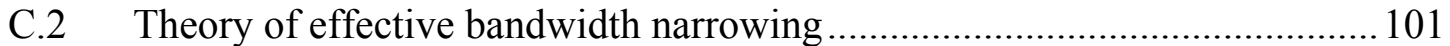

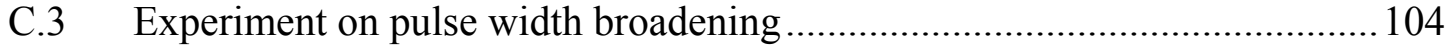

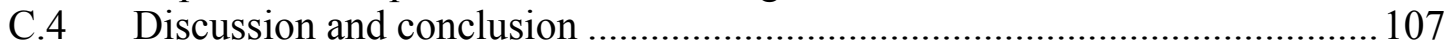

D. Detailed analysis of wide-field two-photon microscopy and wide-field SSTF

D.1 Conventional wide-field TPEF derivation.................................................... 110

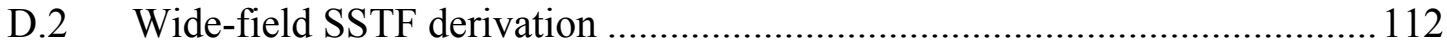

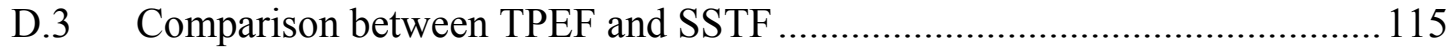

$\begin{array}{ll}\text { E. Gaussian beam propagation } & 118\end{array}$

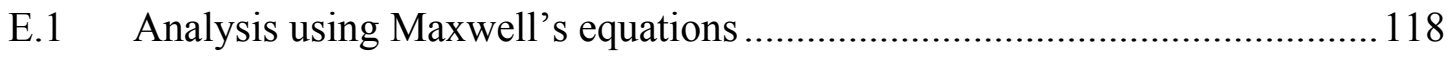

E.2 Analysis using the Fresnel diffraction equation ............................................. 120

F. Detailed analysis of the temporal broadening of a Gaussian pulse due to group-delay dispersion $\quad 124$ 


\section{LIST OF FIGURES}

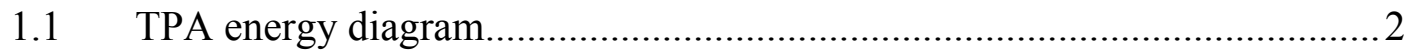

$1.2 \quad$ SSTF setup and beam profiles ……………….........................................

1.3 Wide-field SSTF theoretical axial profiles ...............................................

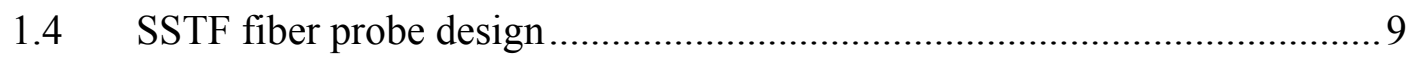

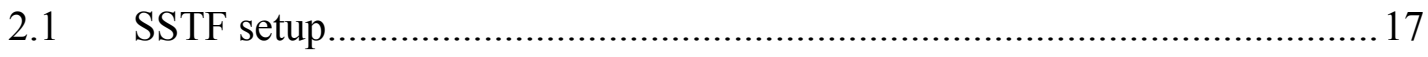

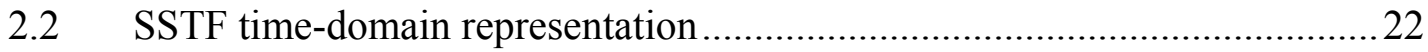

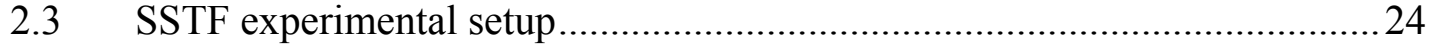

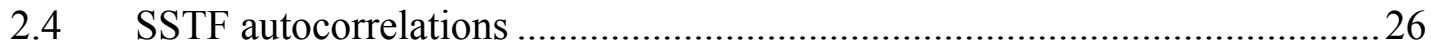

$2.5 \quad$ Pulse width versus axial position .............................................................2 27

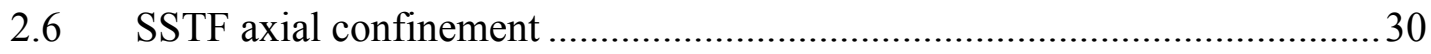

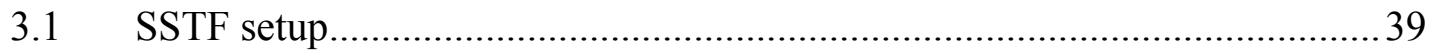

3.2 SSTF axial scanning limit ....................................................................... 43

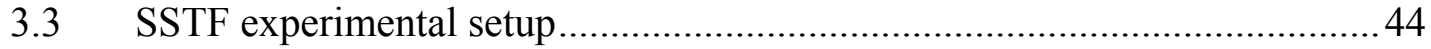

3.4 SSTF axial shift of the temporal focus versus GDD ………………….........4

3.5 Equivalence between GDD and mechanical scans........................................48

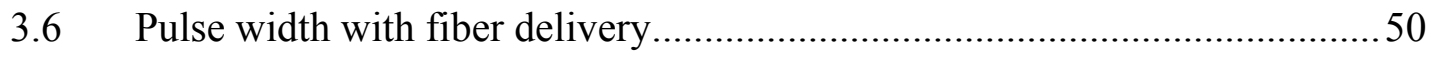

3.7 Axial scanning with wide-field and line-scanning SSTF .............................52

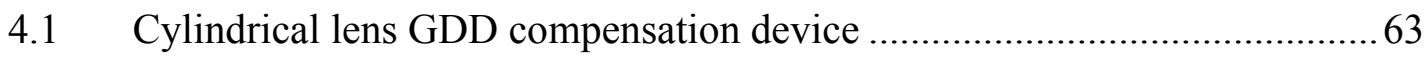

4.2 Pulse width versus rotation angle of cylindrical lens ......................................67

4.3 Autocorrelations at minimum and maximum cylindrical lens curvature ......68

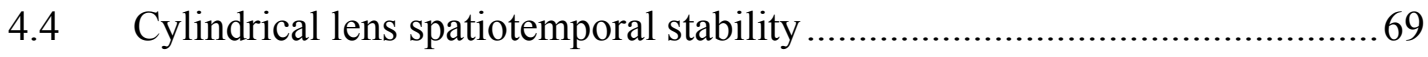

5.1 Piezo bimorph mirror GDD compensation device .........................................77

5.2 GDD versus voltage applied to piezo bimorph mirror .................................... 80

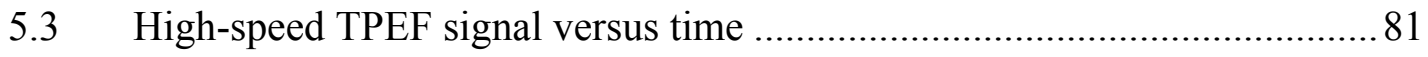




\section{LIST OF FIGURES (CONTINUED)}

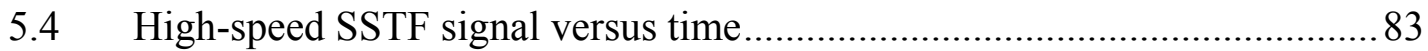

5.5 High-speed SSTF: GDD and mechanical scans ........................................... 84

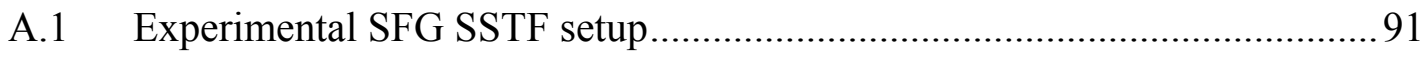

A.2 SFG SSTF excited spectrum versus position and wavelength ......................94

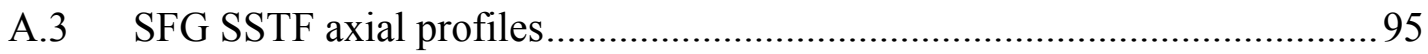

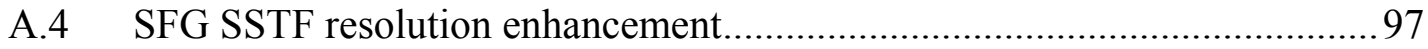

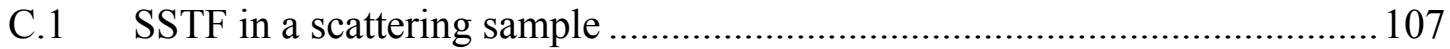

C.2 Pulse width versus NA in a scattering sample................................................ 109

C.3 SSTF experimental setup for measuring pulse widths ................................... 110

C.4 Pulse width versus NA in samples with and without scatterers ..................... 112

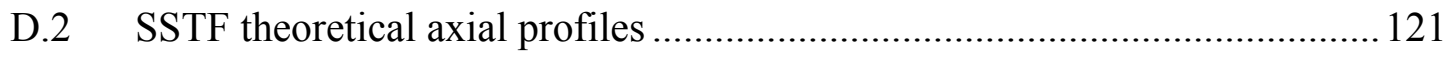




\section{LIST OF TABLES}

2.1 Comparison between conventional TPEF and SSTF ............................. 30 


\section{LIST OF ABBREVIATIONS}

2D, 3D

STORM

PALM

OCT

VIPA

FWHM

TPE

TPEF

TPA

GDD

LCD

SVEA

TEM

SSTF

SBR

PMT

SFG

SHG

MPM

2C2P

OPO

SSMF

SLM

$\mathrm{AOM}$
Two, three dimensional

Stochastic optical reconstruction microscopy

Photoactivated localization microscopy

Optical coherence tomography

Virtually imaged phased array

Full width at half maximum

Two-photon excitation

Two-photon excited fluorescence

Two-photon absorption

Group-delay dispersion

Liquid crystal display

Slowly varying envelope approximation

Transverse electromagnetic

Simultaneous spatial and temporal focusing

Signal-to-background ratio

Photomultiplier tube

Sum-frequency generation

Second harmonic generation

Multiphoton microscopy

Two-color two-photon

Optical parametric oscillator

Standard single mode fiber

Spatial light modulator

Acousto-optic modulator 


\section{PREFACE}

\section{Historical perspectives}

What makes physics, specifically optics, fascinating is that it is constantly growing and evolving. Its history spans religions, continents, and languages. In the early history of optics ${ }^{1}$, we read about mirrors in the Bible (Exodus 38:8) [1] and refraction in Plato's Republic [2, 3]. Abu Ali Hasan Ibn al-Haitham (known in English as Alhazen, the father of modern optics, 965-1040 [2]), furthered the study of optics during the Dark Ages with his understanding of the human eye, reflection, and refraction [4]. Leonardo da Vinci (1452-1519) wrote of the camera obscura, a way of forming images using only a pinhole [2]. Alhazen's understanding of refraction led to the telescope, of which Galileo Galilei (1564-1642) was one of the first users. Then, Zacharias Janssen (1588-1632) created the first microscope, practically an inverted telescope. The mathematical details behind these advances were then detailed by Willebrord Snel (1591-1626) and René Descartes (1596-1650), who collectively formed what is now known as the law of refraction. The law of reflection was finally understood by Pierre de Fermat (1601-1665) with his principle of least time. This insight led to Isaac Newton (1642-1727) making a compact reflecting telescope [5]. Then, Christiaan Huygens (1629-1695) gave us the wave theory of light. Using this wave theory, Augustin Jean Fresnel (1788-1827) developed a formulation for diffraction and for the now common transmission and reflection coefficients [6]. Finally, the concept of waves brings us to James Clerk Maxwell (1831-1879), whose famous equations relate light to electricity and magnetism (see Appendix E) [7].

Related somewhat more directly to the work in this dissertation, Albert Einstein (1879-1955) introduced the concept of the photon and postulated the requirements for both lasing and two-photon absorption [8]. Although he is famous

\footnotetext{
${ }^{1}$ This brief list follows the chronology of Chapter 1 in Ref. [2].
} 
for his work on relativity, Einstein won the Nobel prize in 1921 for his work on light and the photoelectric effect. Maria Göppert-Mayer (1906-1972) formulated the quantum mechanical transitions of two-photon absorption [9], and she won the Nobel prize in 1963 for her work describing the shells of the atom [10]. Using Einstein's equations, Charles Townes invented the maser (microwave amplification by the stimulated emission of radiation) which led to the laser (light amplification by the stimulated emission of radiation) [11] and ultimately the Nobel prize. The invention of ultrafast lasers in the late 1980's led to the first demonstration of two-photon microscopy in 1990 by Winfried Denk, James Strickland, and Watt W. Webb [12], almost sixty years after Maria Göppert-Mayer wrote her thesis. This work was fully formulated by Chris $\mathrm{Xu}$, my advisor, while working for Webb at Cornell University [13]. Note that the major contributors to two-photon microscopy in the early twentieth century (Einstein, Göppert-Mayer, and Townes) all received the Nobel prize. As the principal investigator of the seminal work on two-photon microscopy, I expect to see Watt W. Webb on the short list for the next one. Clearly, I am following in the footsteps of giants.

\section{Why study optics?}

Why do some people think I make eyeglasses if I say I work in optics? Why do many people shutter when hearing that you are a physicist? The typical response is, “Oh, I hated physics in high school." With high school physics no longer a required course, the number of Americans studying physics has been far too few.

There is something special about studying physics: you end up learning about everything. You can understand all the latest electronics technology, including how LCD TV's work, how to fix a computer, or even how to repair a broken doorbell. Although employers do not always agree, graduate school tries to convince physics 
Ph.D. students that they have the skills to tackle any project they want. For example, I look at my wife's work in the field of landscape architecture where they still do design drawings by hand but have to submit the official plans electronically. Why not combine current $3 \mathrm{D}$ rendering technology into the design process? Why did CNN have an interactive touch-screen TV for just zooming in and out of Google Maps to document the 2008 presidential election? It would make much more sense to make the touch-screen capabilities useful, including as a high-tech drawing table for landscape architects and architects. Why not even incorporate new 3D printer technology into making models? This would allow for rapid 3D visualization of design layouts. Although I am sure these ideas are not new (some are already being implemented in the architecture field), this thought process flows naturally when studying physics.

This lack of cross-discipline communication stifles creativity and innovation. This problem is evident even within the physics and optics communities. My advisor, Dr. Chris $\mathrm{Xu}$, had an illustrious career at Bell Labs by using known microwave communication phenomena to discover their optical equivalent. Dr. Jim van Howe, a former group member, took normal optical phenomena in the spatial domain and translated them to the frequency domain using established optical communication technology. I'm convinced that it sometimes takes someone from an outside field to make large-scale leaps in innovation. For example, when NASA wanted to transmit pictures back from space, managers at Bell Labs asked its brilliant employees from many disciplines for ideas. Within two days, the concept of the CCD camera was born. Only recently do we see physicists and computer scientists working in biology and even finance. As mentioned in C.P. Snow's “Two Cultures" lecture in 1959, the lack of interdisciplinary understanding and cooperation has impeded technological advancements that could "eliminate poverty and inequality" [14]. 


\section{Optics and Traffic}

When you immerse yourself in optics long enough, you start to see it all around you. After many trips between Buffalo and Ithaca, I have begun to see optical phenomena in traffic. Having taken a transportation engineering course from the department of civil engineering, I learned that traffic is designed to travel through a dense city grid of controlled intersections (traffic signals) in groups called platoons. This phenomenon easily translates to optical pulses. The gating provided by the traffic signals can be compared to a shutter or a modelocking mechanism in a pulsed laser. Now imagine that these cars have left the downtown area for a divided highway with multiple passing lanes. As the different cars travel at different speeds, one can imagine then that this platoon will break apart: ultimately the faster cars will move to the front of the platoon, and the slower cars will creep toward the back. After a long enough distance, the cars will separate even further until what once was a platoon is now completely spread out along the highway. This phenomenon also happens with optical pulses. Multiple wavelengths are required to generate an optical pulse, and because the refractive index is different for each wavelength, these wavelengths all travel at different speeds. This phenomenon in optics is called dispersion, and this is why optical pulses will spread out after traveling long distances (e.g., in optical fiber, see Appendix F).

Another simple optical phenomenon can be explained with cars: refraction. Recall that the refractive index for a particular wavelength determines how fast that wavelength can travel in a certain medium. Say a car is traveling on a road made of asphalt, but it has to move over to the shoulder to avoid an obstacle. If the shoulder is composed of loose gravel, then the car cannot move as fast. If the two right wheels of the vehicle are on the loose gravel, and the left wheels are on the asphalt, then it will 
feel like the gravel is grabbing the wheel and forcing the car off the road. This is how the refraction of light at an interface works. If a laser beam traveling in air enters a pool of water, then the light ray will bend toward the normal. This is because the refractive index of the water is higher than that of the air, causing the light to travel slower.

Much can be learned about telecommunication within this paradigm. For example, say that the highway with the platoon of cars mentioned earlier has all its lanes blocked by slow-moving trucks. The cars will then bunch up and will travel without the platoon breaking apart. This is like a soliton in telecommunications, where the dispersion of an optical pulse can be compensated by nonlinearities. The total bandwidth that a wire or optical fiber can carry is similar to the volume of cars that a road can accommodate. Because there is a required amount of separation between cars to be able to travel safely, there are only so many cars that can be on the road before they have to slow down. With too many cars, the flow will eventually stop. Similarly, there is a maximum data rate in optical communications that is determined by the bandwidth and the temporal width of the pulses. If you were to increase the number of lanes on a highway, you can accommodate more cars. Similarly, if you were to increase the number of channels by encoding information on multiple frequencies, the data rate can be increased.

One can actually see a literal form of optical communication on the highway when someone illuminates their brake lights. The lead driver sees something in the road and hits the brakes, and the cars behind that driver will still be braking hours later. The signal for other drivers to brake propagates like a wave in the backward direction [15]. Even though the driver who first braked is now traveling along unimpeded, the location of the back of the newly formed platoon is now slowly traveling backward. Correct spacing of vehicles is required to cushion the impact of 
such slowdowns. If the volume of cars is too high, then eventually drivers will be forced to stop and wait for the wave to pass by. Similarly in telecommunications, buffering is required when the data arrives too quickly to be immediately processed. I hope this dissertation is only the beginning of my quest to bring a love for physics to everyone. 


\section{REFERENCES}

1. The Bible. New International Version.

2. E. Hecht, Optics (Addison-Wesley, New York, 2002).

3. Plato, "The Republic," in Plato's The Republic, B. Jowett, ed. (The Modern Library, New York, 1941).

4. Alhazen, Kitab al-manā̄ir (National Council for Culture, Arts, and Letters, Kuwait, 1983).

5. I. Newton, Philosophiae naturalis principia mathematica (Royal Society, London, 1726).

6. A. J. Fresnel, Explication de la refraction dans le systeme des ondes (1821).

7. J. C. Maxwell, "A dynamical theory of the electromagnetic field," Philos. Trans. Roy. Soc. 155, 459-512 (1865).

8. A. Einstein, "Creation and Conversion of Light," Ann. Physik 17, 132-148 (1905).

9. M. Göppert-Mayer, "Elementary processes with two quantum jumps," Ann. Physik 9, 273-294 (1931).

10. B. R. Masters, and P. T. C. So, eds. Handbook of Biomedical Nonlinear Optical Microscopy (Oxford University Press, New York, 2008).

11. A. L. Schawlow, and C. H. Townes, "Infrared and Optical Masers," Physical Review 112, 1940 (1958).

12. W. Denk, J. H. Strickler, and W. W. Webb, "2-Photon Laser Scanning Fluorescence Microscopy," Science 248, 73-76 (1990).

13. C. $\mathrm{Xu}$, and W. W. Webb, "Measurement of two-photon excitation cross sections of molecular fluorophores with data from 690 to 1050 nm," J. Opt. Soc. Am. $B$ 13, 491 (1996). 
14. J. Rennie, "Inspirational Orbits," in Scientific American (Scientific American, Inc., New York, 2009), p. 8.

15. C. S. Papacostas, and P. D. Prevedouros, Transportation Engineering and Planning (Prentice Hall, Upper Saddle River, NJ, 2001). 


\section{CHAPTER 1}

\section{INTRODUCTION}

\section{$1.1 \quad$ Multiphoton microscopy}

Multiphoton microscopy (MPM) is a powerful tool for imaging biological samples due to its ability to perform optical sectioning $[1,2]$. MPM yields many advantages over standard one-photon imaging: a deeper penetration depth due to longer wavelength excitation $[3,4], 3 \mathrm{D}$ confinement of the focal volume, and reduced photodamage [5]. Also, the ability to selectively target cells with attached fluorophores makes MPM a good candidate for biomedical imaging applications. By performing non-invasive imaging of tissues, MPM can act as a form of optical biopsy for cancer diagnostics. For example, sub-cellular resolution imaging at a depth of 1 $\mathrm{mm}$ has been achieved in mouse brain using both a regenerative amplifier [4] and a long-wavelength excitation source (optical parametric oscillator) [6]. In this chapter, I will detail our efforts to further improve the utility of multiphoton excitation, specifically through the technique of simultaneous spatial and temporal focusing.

\subsection{Two-photon microscopy}

In two-photon excited fluorescence (TPEF) microscopy, the fluorophore is excited by photons that carry less than the amount of energy required for absorption. Intuitively, the first photon excites the molecule to a virtual intermediate state, and the second excites it to its upper energy level (Figure 1.1) [7]. These photons must arrive simultaneously in order to excite the fluorophore. This is unlikely, however, because the two excitation photons must arrive at the same place at the same time. Ultrashort pulsed lasers and high numerical aperture objectives are used to maximize the likelihood of this absorption event, both by confining the photons to a short pulse in 


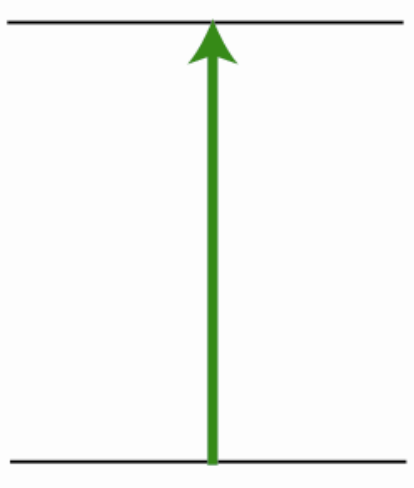

(a) one-photon absorption

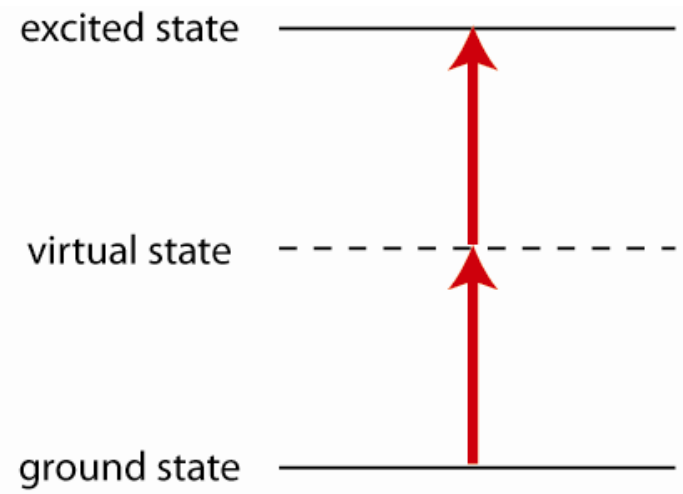

(b) two-photon absorption

Figure 1.1: Energy diagram for (a) one-photon and (b) two-photon absorption. 
time and a small volume in space. Because TPEF is a second-order process, the fluorescence signal depends quadratically on the excitation intensity [7], thereby confining the signal to the focal volume of the objective and thus substantially decreasing the out-of-focus background $[1,2,8]$. The functional dependence of the average two-photon excited fluorescence (TPEF) is:

$$
\langle\operatorname{TPEF}(\mathbf{r}, t)\rangle \propto \frac{\left\langle I^{2}(\mathbf{r}, t)\right\rangle}{f \cdot \tau}
$$

where $I(\mathbf{r}, t)$ is the intensity of the excitation beam, $f$ is repetition rate of the pulses, and $\tau$ is the temporal pulse width. A normal excitation beam has a constant repetition rate and temporal pulse width along the axial direction. Thus, the spatial, not temporal, focusing due to the objective lens determines the axial confinement achieved in conventional TPEF microscopy.

\subsection{Simultaneous spatial and temporal focusing ${ }^{2}$}

Many efforts have been made to further enhance the axial confinement in MPM [9]. Clearly, if the constants in Eq. (1.1) were to vary as a function of axial position, another degree of confinement could be obtained. We demonstrate varying the pulse width as a function of axial position, known as simultaneous spatial and temporal focusing (SSTF). Axial confinement can be obtained by creating a temporal focus where the shortest pulse width only occurs at the geometric (or spatial) focal plane $[10,11]$. SSTF works by spatially separating the frequencies of a short pulse with a grating, collimating these beams with a lens, and then recombining them with an objective lens [10]. The individual frequency components are spatially separated by the grating and recombine only within the focal region of the objective lens. Thus the pulse width is shortest only at the focal plane, creating a "temporal focus." One

\footnotetext{
${ }^{2}$ This summary has been reproduced in part from Optics Communications, 281, p. 1805, 2008.
} 
can also describe this as the grating being imaged to the focal plane of the objective lens. By Fermat's principle, the light rays emerging from the grating travel the same optical path length to the focal plane, and so a short pulse at the grating will be imaged as a short pulse at the focus. This is not true for points outside of the focus, however, for different rays will not add in phase, thus broadening the pulse [10-12].

Figure 1.2(a) shows a schematic of a typical SSTF setup. There are two imaging modalities for SSTF: wide field and line scanning. In conventional wide-field imaging, the beam incident upon the input focal plane of the objective lens is small, resulting in a loosely focused spot at the focal plane. In the wide-field SSTF case, however, the beam shape at the input focal plane of the objective is a thin line, where the width in the $x$-direction is the spreading of the monochromatic components due to the grating, and the height in the $y$-direction is the monochromatic beam size $s$ (see Figure 1.2(b)). Wide-field illumination in SSTF is achieved by using a spherical lens as the collimating lens to focus the spatially chirped beam to a small height $s$ in the $y$ dimension at the input focal plane of the objective lens. Because each monochromatic beam is small, the objective lens will not focus the monochromatic beams tightly, creating a large spot at the focal plane (Figure 1.2(b)). In contrast with conventional imaging, however, wide-field imaging with SSTF allows for optical sectioning due to the evolution of the temporal pulse width within the sample. In the SSTF linescanning case, the beam has a circular cross-section at the input focal plane of the objective lens, such that the $y$-component of each monochromatic beam is large, but the $x$-component of each monochromatic beam remains the small width $s$. Thus, each monochromatic beam will focus to a large $x$-dimension but a small $y$-dimension, forming a line at the focus of the objective lens (Figure 1.2(c)). By using a 1D scanner to sweep the line across the sample, 2D images can be obtained [13]. 
(a)

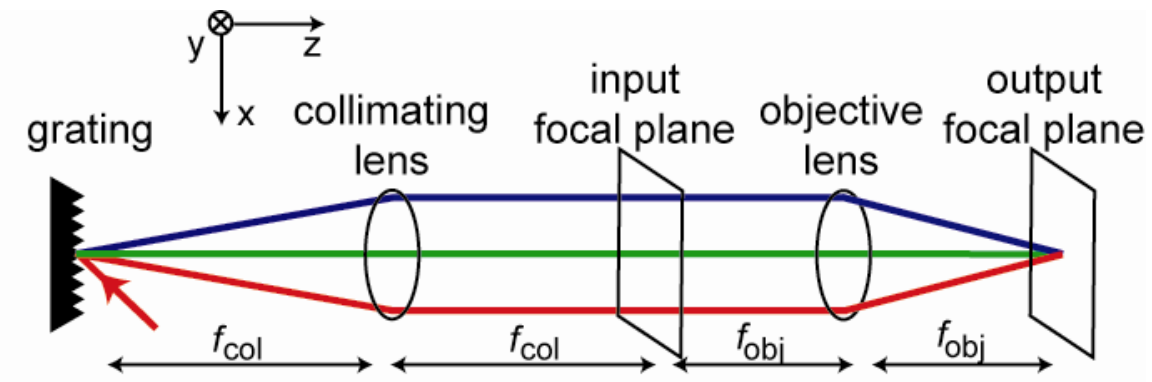

(b)

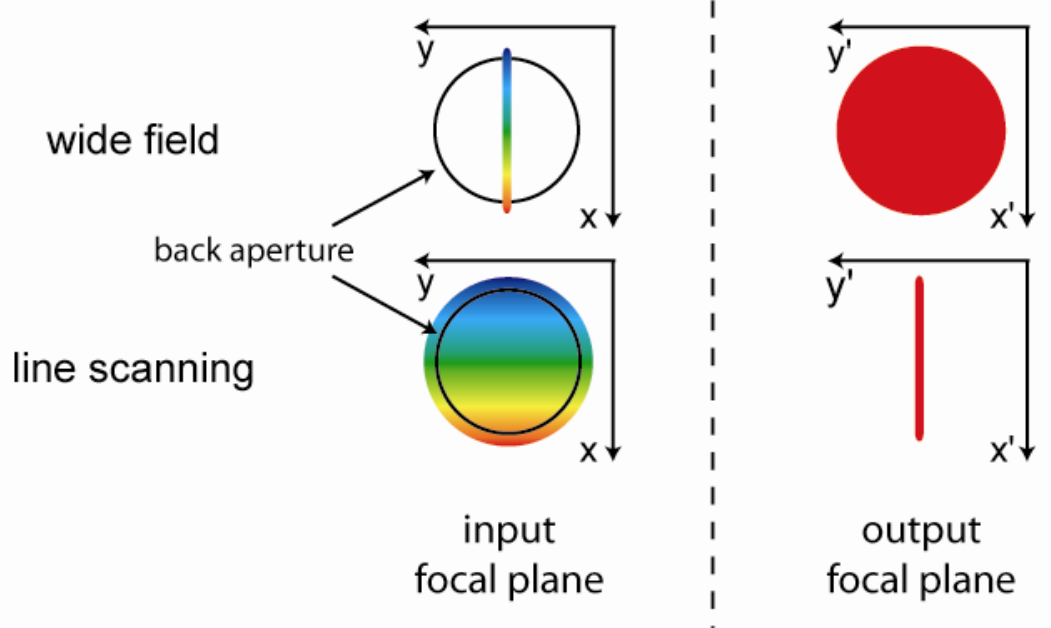

Figure 1.2: (a) A typical SSTF setup. (b) Beam profile for wide-field SSTF. On the left is a cross-section of the beam at the input focal plane of the objective. On the right represents the beam shape at the focal plane. (c) Beam profile for line scanning SSTF. 


\subsection{Comparison between TPEF and SSTF}

This section will compare the axial profile and total signal of conventional TPEF microscopy and line-scanning SSTF with Gaussian beams. For detailed derivations, please see Appendix D. The two-photon excitation (TPE) signal as a function of axial position $z$ can be expressed as:

$$
T P E_{\text {conventional }}(z)=\frac{C}{\left(1+\left(\frac{z}{z_{R}}\right)^{2}\right)}
$$

For line-scanning SSTF, a similar expression can be obtained:

$$
T P E_{\mathrm{SSTF}}(z)=\frac{C}{\sqrt{1+\left(\frac{z}{z_{R}}\right)^{2}}} \frac{1}{\sqrt{1+\left(\frac{z}{z_{M}}\right)^{2}}}
$$

In the limit that the Rayleigh lengths for spatial focusing in the $y$-dimension $\left(z_{\mathrm{M}}\right)$ and temporal focusing in the $x$-dimension $\left(z_{\mathrm{R}}\right)$ match, then Eqs. (1.2) and (1.3) are identical. Therefore, temporal focusing replaces spatial focusing in one dimension. For wide-field SSTF, the Rayleigh length in the $y$-dimension $\left(z_{\mathrm{M}}\right)$ will be essentially infinite, eliminating the second square root term. On the other hand, conventional wide-field imaging will have no confinement at all. Because the two formulae share the same coefficients, one can imagine that wide-field SSTF is carved out of its corresponding wide-field TPEF profile (Figure 1.3). Unfortunately, this means that the peak axial SSTF signal matches that of a wide-field beam, not a point-scanning beam. Therefore, the total SSTF signal is an order of magnitude lower in comparison to point-scanning TPEF. 


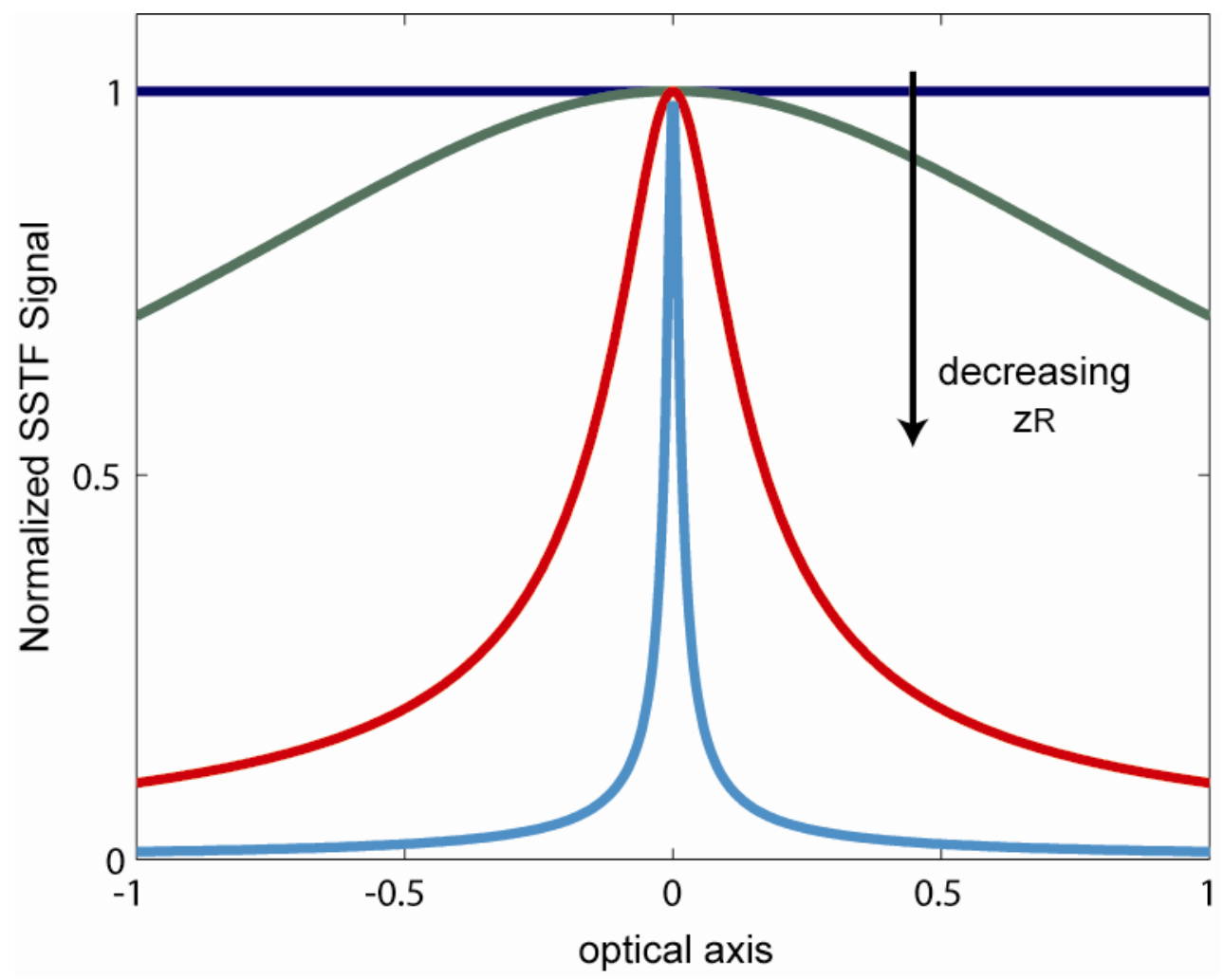

Figure 1.3: Wide-field SSTF signal as function of axial position $\mathrm{z}$ for different values of $z_{\mathrm{R}}$. In the wide-field two-photon imaging case $\left(z_{\mathrm{R}}\right.$ infinite), there is no confinement, and the axial profile is flat (black curve). As the Rayleigh length is decreased, the SSTF effect carves out a square-root Lorentzian profile with a peak value equal to that of the conventional two-photon wide-field imaging case. 


\subsection{Applications of SSTF}

Because SSTF can provide wide-field illumination, high-speed two-photon excitation can be achieved without the need for scan mirrors [10]. Unlike conventional wide-field imaging, wide-field SSTF provides optical sectioning. Recently, this has been applied to photoactivated localization microscopy (PALM), or stochastic optical reconstruction microscopy (STORM), for biological imaging beyond the diffraction limit with a large field of view [14]. Normally, PALM provides a super-resolution of less than $50 \mathrm{~nm}$, but it applies only in the lateral dimension and only at the surface of the sample. In this case, SSTF confines the excitation volume of PALM to a $5 \mu \mathrm{m}$ axial width.

Another key characteristic of SSTF is that the presence of group delay dispersion (GDD) in the beam path will axially shift the temporal focus (Chapter 3). By rapidly varying the GDD, one can achieve high-speed axial scanning. A detailed derivation of axial scanning by tuning the GDD is provided in Appendix B. We have developed two techniques for high-speed GDD tuning by using a cylindrical lens (Chapter 4) and a piezo bimorph mirror (Chapter 5). Both of these techniques are low cost, high speed, high power, and broad bandwidth solutions, characteristics that are not available together in any one commercial product.

Axial scanning of the temporal focus with GDD tuning in an SSTF setup enables the realization of a high-speed axial-scanning endoscopic probe with no moving parts at the distal end of the fiber. When the probe excites two-photon fluorescence, the collected emission spectrum acts as a signature for detecting cancerous cells, allowing for a $z$-scanning optical biopsy of tissue. We have designed a SSTF fiber probe to incorporate this high-speed remote axial scanning (Figure 1.4). Our current design requires a transmission grating, which when bonded with off-theshelf SF11 prisms will operate at Littrow, allowing for an inline geometry. The grating 


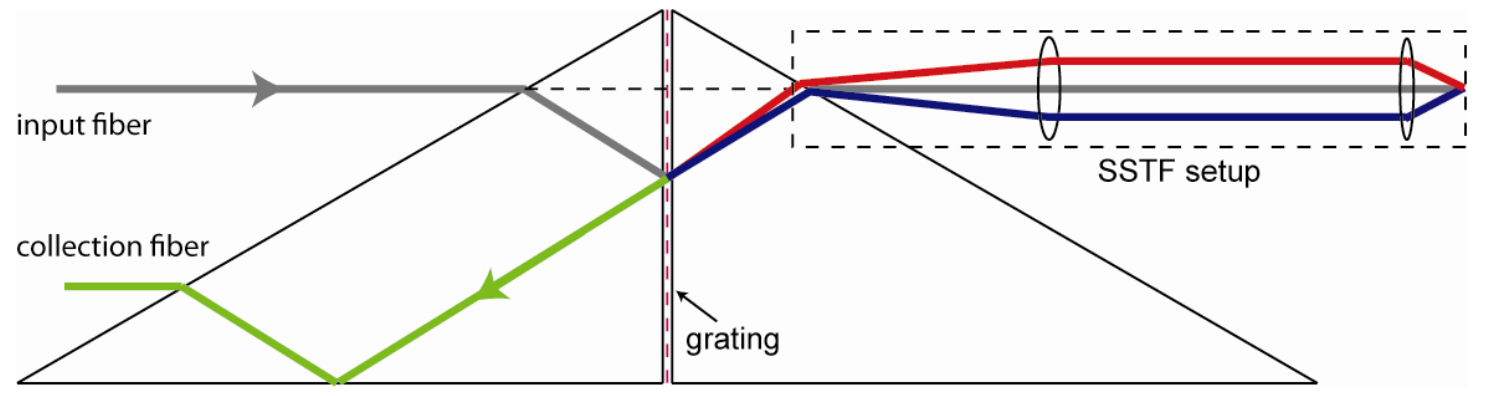

Figure 1.4: $\quad$ Proposed design for an SSTF fiber probe (to scale). 
also acts as a dichroic mirror in that it separates the excitation and emission beam paths. Also, if the efficiency of the transmission grating is sufficiently narrow band, alternate excitation regimes for reflectance, optical coherence tomography (OCT), and long-wavelength excitation for two-photon imaging can be delivered. Our current design is $15 \mathrm{~mm}$ long and $4 \mathrm{~mm}$ wide, and provides $10 \mu \mathrm{m}$ FWHM resolution and a scan range of $100 \mu \mathrm{m}$. In summary, this probe will act as a 1D axial scanning probe that can simultaneously record the emission spectrum as a function of position, enabling diagnostics of different layers of tissue.

Future applications of SSTF include further investigation of wide-field twophoton imaging. The applicability of SSTF to a highly scattering sample has been studied and is available in Appendix C. A future study of SSTF in highly scattering samples will include pre-compensating for the effective spectral-shaping of the excitation pulse and as well as using the temporal pulse width to determine the scattering properties of the sample. Also, the compensation of higher-order dispersion terms may be able to compensate for higher-order focusing aberrations (e.g., spherical aberration). Another application of SSTF is to perform sum-frequency generation (SFG) imaging with a spectrally shaped excitation beam (Appendix A). For a fluorophore with a narrow absorption spectrum or a second-harmonic generation (SHG) sample combined with a narrow bandpass emission filter, an SSTF setup can effectively become a two-color two-photon setup with a single laser source by physically blocking the center of the spectrum. We have demonstrated an order of magnitude enhancement of the axial confinement in such an SFG SSTF setup. Further work will include more sophisticated ways of spectrally shaping the beam, including using nonlinearities in a fiber or spatial light modulators $[15,16]$. Finally, our work has focused on temporal focusing due to diffraction in one dimension, but new work in spectrally encoded endoscopy shows the ability to spread the individual frequency 
components into a two-dimensional array using an Echelle grating or a virtually imaged phased array (VIPA) [17]. This new 2D spectral grid pattern might not allow for remote axial scanning due to its lack of cylindrical symmetry. However, the use of a circular grating would preserve such symmetry and still generate temporal focusing in two dimensions.

In summary, this dissertation illustrates the development and understanding of simultaneous spatial and temporal focusing. Chapter 2 details both the frequency- and time-domain understandings of SSTF, the evolution of the pulse width along the optical axis, and the axial confinement of the SSTF signal. In Chapter 3, I describe the effects of GDD on SSTF, specifically its ability to axially scan the temporal focus and its applicability to fiber probes. In Chapter 4, I demonstrate a novel technique for GDD tuning by rotating a cylindrical lens in a folded 4-f grating pair setup. In Chapter 5, I describe another technique for GDD tuning using a piezo bimorph mirror. Using this new device, I demonstrate high-speed SSTF axial scanning. The appendices consist of projects not directly related to this dissertation, including using SSTF with a shaped spectrum to excite SFG (Appendix A) and an analysis of the effect of scattering on the temporal focus (Appendix C). Finally, for the benefit of the reader, Appendices B and D fully derive the SSTF focusing equations, and Appendices $\mathrm{E}$ and $\mathrm{F}$ detail the spatial diffraction and temporal pulse broadening of a Gaussian pulse. 


\section{REFERENCES}

1. W. Denk, J. H. Strickler, and W. W. Webb, "2-Photon Laser Scanning Fluorescence Microscopy," Science 248, 73-76 (1990).

2. W. R. Zipfel, R. M. Williams, and W. W. Webb, "Nonlinear magic: multiphoton microscopy in the biosciences," Nature Biotechnology 21, 1368-1376 (2003).

3. F. Helmchen, and W. Denk, "Deep tissue two-photon microscopy," Nat Meth 2, 932-940 (2005).

4. P. Theer, M. T. Hasan, and W. Denk, "Two-photon imaging to a depth of 1000 microns in living brains by use of a Ti : A12O3 regenerative amplifier," Optics Letters 28, 1022-1024 (2003).

5. B. R. Masters, and P. T. C. So, eds. Handbook of Biomedical Nonlinear Optical Microscopy (Oxford University Press, New York, 2008).

6. D. Kobat, M. E. Durst, N. Nishimura, A. Wong, C. B. Schaffer, and C. Xu, "Comparison of two-photon imaging depths with 775-nm and 1280-nm excitation," Optics Express in press (2009).

7. C. Xu, "Cross-Sections of Fluorescent Molecules in Multiphoton Microscopy," in Confocal and Two-Photon Microscopy: Foundations, Applications, and Advances, A. Diaspro, ed. (Wiley-Liss, Inc., New York, 2002), pp. 75-100.

8. J. P. Ying, F. Liu, and R. R. Alfano, "Effect of scattering on nonlinear optical scanning microscopy imaging of highly scattering media," Applied Optics 39, 509-514 (2000).

9. M. E. Durst, G. Zhu, and C. Xu, "Simultaneous spatial and temporal focusing in nonlinear microscopy," Optics Communications 281, 1805 (2008).

10. D. Oron, E. Tal, and Y. Silberberg, "Scanningless depth-resolved microscopy," 
Optics Express 13, 1468-1476 (2005).

11. G. Zhu, J. van Howe, M. Durst, W. Zipfel, and C. Xu, "Simultaneous spatial and temporal focusing of femtosecond pulses," Optics Express 13, 2153-2159 (2005).

12. E. Tal, and Y. Silberberg, "Transformation from an ultrashort pulse to a spatiotemporal speckle by a thin scattering surface," Optics Letters 31, 3529-3531 (2006).

13. E. Tal, D. Oron, and Y. Silberberg, "Improved depth resolution in video-rate line-scanning multiphoton microscopy using temporal focusing," Optics Letters 30, 1686-1688 (2005).

14. A. Vaziri, J. Tang, H. Shroff, and C. V. Shank, "Multilayer three-dimensional super resolution imaging of thick biological samples," Proceedings of the National Academy of Sciences 105, 20221-20226 (2008).

15. E. Papagiakoumou, V. de Sars, V. Emiliani, and D. Oron, "Temporal focusing with spatially modulated excitation," Opt. Express 17, 5401 (2009).

16. E. Papagiakoumou, V. de Sars, D. Oron, and V. Emiliani, "Patterned twophoton illumination by spatiotemporal shaping of ultrashort pulses," Opt. Express 16, 22047 (2008).

17. K. Goda, K. K. Tsia, and B. Jalali, "Serial time-encoded amplified imaging for real-time observation of fast dynamic phenomena," Nature 458, 1145-1149 (2009). 
CHAPTER 2

SIMULTANEOUS SPATIAL AND TEMPORAL FOCUSING IN NONLINEAR MICROSCOPY ${ }^{3}$

\begin{abstract}
Simultaneous spatial and temporal focusing (SSTF), when combined with nonlinear microscopy, can improve the axial excitation confinement of wide-field and line-scanning imaging. Because two-photon excited fluorescence depends inversely on the pulse width of the excitation beam, SSTF decreases the background excitation of the sample outside of the focal volume by broadening the pulse width everywhere but at the geometric focus of the objective lens. This chapter theoretically describes the beam propagation within the sample using Fresnel diffraction in the frequency domain, deriving an analytical expression for the pulse evolution. Also, we theoretically define the axial confinement for line-scanning SSTF imaging using a time-domain understanding and conclude that line-scanning SSTF is similar to the temporally-decorrelated multifocal multiphoton imaging technique. Experiments on the temporal focusing effect and its axial confinement are presented. The temporal focusing effect in SSTF essentially replaces the focusing of one spatial dimension in conventional wide-field and line-scanning imaging. Although the best axial confinement achieved by SSTF cannot surpass that of a regular point-scanning system, this trade-off between spatial and temporal focusing can provide significant advantages in applications such as high-speed imaging and remote axial scanning in an endoscopic fiber probe.
\end{abstract}

\footnotetext{
${ }^{3}$ The contents of this chapter have been reproduced from Optics Communications 281, 1805 (2008).
} 


\subsection{Introduction}

Multiphoton microscopy (MPM) has become a powerful tool for imaging biological samples due to its ability to perform optical sectioning $[1,2]$. In twophoton excited fluorescence (TPEF) microscopy, for example, the signal depends quadratically on the excitation intensity, thus substantially decreasing the out-of-focus background $[1,3]$. TPEF also depends inversely on the temporal pulse width of the excitation pulse, but in standard TPEF microscopy, the temporal pulse width is a constant throughout the sample. Thus, the spatial focusing due to the objective lens determines the axial confinement achieved in conventional TPEF microscopy. In this chapter, we introduce simultaneous spatial and temporal focusing (SSTF) in which the pulse width varies as a function of axial position. A temporal focus occurs because when a grating is imaged onto a sample, the different frequency components only overlap within the focal region of the objective lens to yield a short pulse. Outside of the focus, however, different rays will not constructively add in phase, thus broadening the pulse.

In this chapter, we review the derivation of the beam propagation to the focal volume in wide-field SSTF using Fresnel diffraction in the frequency domain (Section 2.1). In Section 2.2, we present a derivation for the axial confinement of line-scanning SSTF in the time domain. In Section 2.3, we review experimental results of the temporal focusing effect and characterize the axial confinement of SSTF. In Section 2.4, we discuss in detail the axial confinement of SSTF in an MPM setup.

\subsection{Theoretical Analysis of SSTF}

\subsubsection{Temporal pulse-width evolution}

Our frequency-domain analytical model for the theoretical understanding of SSTF uses Gaussian beam propagation under the paraxial limit [4]. We assume that 
the input beam profile, $A_{1}(x, \omega)$, at the input focal plane of the objective lens can be written as a superposition of many monochromatic, spatially transform-limited Gaussian beams whose center positions are linearly displaced according to their wavelengths. We further assume that the optical spectrum of the input waveform has no chirp and has a Gaussian spectral profile. For one monochromatic beam with frequency $\omega$, the beam amplitude at the input focal plane of the objective lens is:

$$
A_{1}(x, \omega)=A_{0} e^{-\frac{\omega^{2}}{\Omega^{2}}} e^{-\frac{(x-\alpha \omega)^{2}}{s^{2}}}
$$

where $A_{0}$ is a normalization constant, $\sqrt{2 \ln 2} \cdot \Omega$ is the full-width half maximum (FWHM) of the frequency spectrum of the pulse, $\sqrt{2 \ln 2} \cdot s$ is the FWHM of each monochromatic beam in space, $\alpha$ is a constant proportional to the groove density of the grating and the focal length of the collimating lens [5], and $\alpha \omega$ is the linear displacement of the monochromatic beam of frequency $\omega$.

A typical SSTF setup is shown in Figure 2.1. In order to analytically describe the field at the output, we follow the paraxial approximation to propagate the beam to the focal volume (for detailed calculations, see Appendix B) [6]. The spatiallychirped beam, $A_{1}(x, \omega)$, is incident upon the input focal plane of the objective lens. First, the Fresnel diffraction formula is applied to $A_{1}$ to propagate the beam to the objective lens. After adding the quadratic phase due to the objective lens, the Fresnel diffraction formula is used again to propagate it a distance $z$ toward the focal plane. Fourier transforming back into the time domain, the field distribution at the focal volume is:

$$
A_{2}(x, z, t)=\kappa \cdot e^{-\frac{x^{2}}{s_{2}^{2}}} e^{-\frac{\Omega^{2}}{4(1+\chi)}(t+\gamma x)^{2}}
$$

where

$$
\kappa=A_{0} \Omega \sqrt{\frac{i \pi f}{z_{M}}} \frac{1}{\left[i\left(\frac{f-z}{z_{R}}\right)-1\right]^{\frac{1}{2}}}
$$




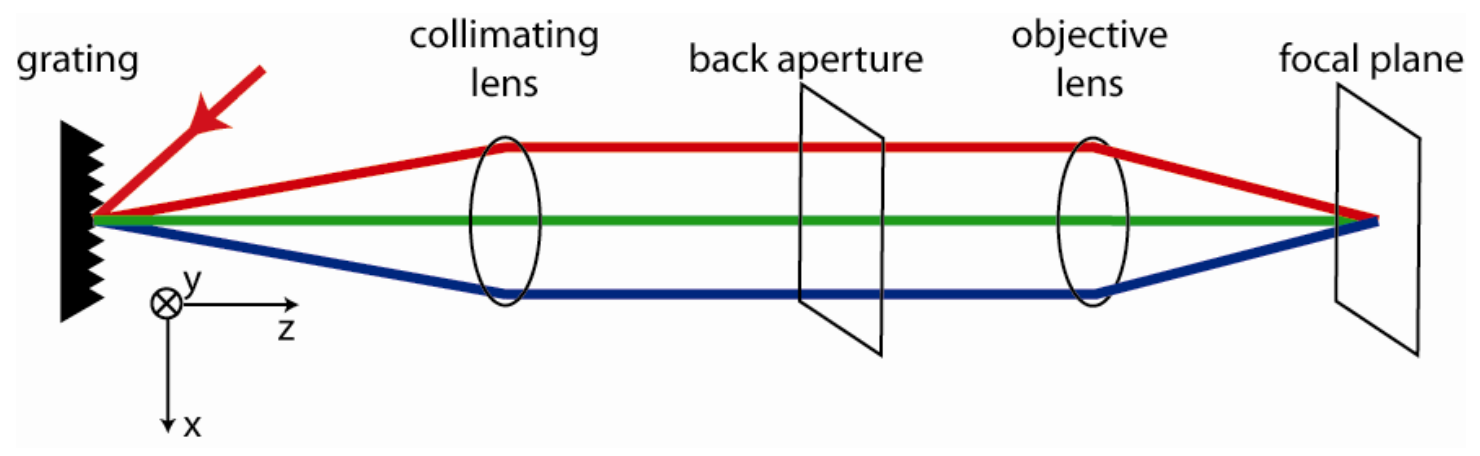

Figure 2.1: A typical SSTF setup. 


$$
\begin{aligned}
\gamma & =\frac{k_{0} \alpha / f}{1+i(z-f) / z_{M}} \\
\chi & =\frac{i(z-f) / z_{B}}{1+i(z-f) / z_{M}}, \\
s_{2}^{2} & =\frac{4 f^{2}}{k_{0}^{2} s^{2}}+i \frac{2(z-f)}{k_{0}}, \\
z_{M}=\frac{2 f^{2}}{k_{0} s^{2}}, z_{R} & =\frac{2 f^{2} / k_{0}}{s^{2}+\alpha^{2} \Omega^{2}}, \text { and } z_{B}=\frac{2 f^{2}}{k_{0} \alpha^{2} \Omega^{2}} .
\end{aligned}
$$

In Eqs. (2.3), $f$ is the focal length of the objective lens, and $z$ is the distance along the optical axis from the objective lens. To simplify calculations, we assume in the derivation of Eq. (2.2) that the wavevector $k$ for each frequency is approximately $k_{0}$, the wavevector of the center wavelength of the pulse, which is valid for pulse widths on the order of $100 \mathrm{fs}$. The FWHM pulse width $\tau$ depends only on the propagation distance $z$ :

$$
\tau(z)=\frac{2 \sqrt{2 \ln 2}}{\Omega} \cdot\left[1+\frac{z_{M}}{z_{B}} \frac{(z-f)^{2}}{(z-f)^{2}+z_{M} z_{R}}\right]^{\frac{1}{2}}
$$

Clearly, the pulse width will be shortest at the geometric focal plane of the objective lens $(z=f)$. Note that the FWHM pulse width of $2 \sqrt{2 \ln 2} / \Omega$ at the focus is the transform-limited value for a Gaussian pulse with a FWHM bandwidth of $\sqrt{2 \ln 2} \cdot \Omega$, indicating that the pulse at the focal point is chirp free. At positions outside of the focus, the temporal pulse width increases quickly, demonstrating the effect of temporal focusing.

\subsubsection{Axial confinement}

Rigorous treatment of the focusing characteristics of pulsed input illumination should be done in the frequency domain. However, under the condition that the input pulse spectrum is narrow, the frequency-domain theory can be replaced by that in the 
time domain, owing to the fact that the difference between the diffraction patterns for different frequencies inside the focal volume is negligible. In this section, an understanding of SSTF axial confinement is developed in the time domain, unifying the ray-optics and wave-optics description of SSTF. This time-domain treatment also provides direct comparison between SSTF and conventional imaging.

We consider line-scanning SSTF and assume that there is no initial chirp, and the beam at the input focal plane of the objective lens is circular in cross-section. When a grating is used to spatially separate the individual frequency components of the beam, their center positions are laterally displaced according to their offset frequency measured from the spectrum center. Following the treatment of Martinez $[5,7]$, when diffraction is taken into account and assuming a chirp-free Gaussian spectral profile, a fast-rotating and a both temporally and spatially-stretched wavefront is formed at the output focal plane of the collimating lens (i.e., the input focal plane of the objective lens, see Figure 2.1). The profile of the beam at the input focal plane of the objective can be calculated in the time-domain by taking the Fourier transform of Eq. (2.1) with the added $y$-component for a line-scanning SSTF setup:

$$
\begin{aligned}
B_{1}(x, y, t) & =D_{0} \int_{-\infty}^{\infty} e^{-\frac{(x-\alpha \omega)^{2}}{s^{2}}} e^{-\frac{y^{2}}{s^{2}+\alpha^{2} \Omega^{2}}} e^{-\frac{\omega^{2}}{\Omega^{2}}} e^{-i \omega t} d \omega \\
& =D_{1} e^{-\frac{x^{2}+y^{2}}{s^{2}+\alpha^{2} \Omega^{2}}} e^{-\frac{t^{2}}{2 \tau_{0}^{2}\left(1+\frac{\alpha^{2} \Omega^{2}}{s^{2}}\right)}} e^{i \frac{\alpha \Omega^{2} \cdot t \cdot x}{s^{2}+\alpha^{2} \Omega^{2}}},
\end{aligned}
$$

where $D_{0}$ is a normalization constant, $D_{1}$ is a constant independent of the time and transverse spatial coordinates, and $\tau_{0}=\sqrt{2} / \Omega$ describes the temporal pulse width. Note that when compared to the case where the stretching of the beam is absent (i.e., $\alpha=0$ ), both the spatial width and temporal width of the incident beam are now stretched by a factor of $\sqrt{1+\alpha^{2} \Omega^{2} / s^{2}}$.

In the time-domain approach, the system temporal response to the input field is instantaneous, i.e. in the reference frame that travels with the pulse (the retarded 
frame), the spatial output pattern of the system at time $t$ is uniquely determined by its spatial input pattern at the input focal plane of the objective at the same time $t$. This implies that the spectral response of the spatial focusing does not vary across the pulse bandwidth. We propagate the field at the input focal plane of the objective, $B_{1}(x, y, t)$, to the output focal plane following the same Fresnel diffraction method used in Section 2.1 except with an additional pupil function [8]. The field distribution inside the focal volume can then be expressed as:

$$
B_{2}(x, y, z, t) \approx D_{2} e^{-\frac{t^{2}}{\tau_{\text {sweep }}^{2}}} h\left(x+v_{\text {sweep }} t, y, z\right)
$$

where

$$
\begin{aligned}
& h(x, y, z)=\iint_{\infty} P\left(x^{\prime}, y^{\prime}\right) e^{-\frac{x^{\prime 2}+y^{\prime 2}}{s^{2}+\alpha^{2} \Omega^{2}}} e^{i k_{0}\left(\frac{x x^{\prime}+y y^{\prime}}{f}+z \frac{x^{\prime 2}+y^{\prime 2}}{2 f^{2}}\right)} d x^{\prime} d y^{\prime}, \\
& v_{\text {sweep }}=\frac{f}{k_{0}} \frac{\alpha \Omega^{2}}{s^{2}+\alpha^{2} \Omega^{2}}, \\
& \tau_{\text {sweep }}=\tau_{0} \cdot \sqrt{1+\frac{\alpha^{2} \Omega^{2}}{s^{2}}} .
\end{aligned}
$$

Here, $D_{2}$ is a constant independent of the time and spatial coordinates, and $P\left(x^{\prime}, y^{\prime}\right)$ is the pupil function of the objective. We note that $h(x, y, z)$ defined in Eq. (2.7) is the standard point-spread function but with the back aperture partially filled due to the effect of the finite size of the spatially-chirped beam.

Equation (2.6) presents a clear physical picture of the SSTF technique. At the focal plane, the field distribution measured at any instantaneous time $t$ is a focused point that is determined by the point-spread function $h(x, y, z)$ associated with a finite beam size $\sim \sqrt{s^{2}+\alpha^{2} \Omega^{2}}$ at the input focal plane of the objective. Different from a regular point-focusing system, however, this focused point sweeps rapidly at the speed $v_{\text {sweep }}$ along the $x$-direction. This ultrafast point-sweeping process will complete in a time duration of $\sim \tau_{\text {sweep }}$, which corresponds to the temporal stretching of the spatiallychirped beam at the input focal plane of the objective lens. Intuitively, this is the 
result of different incident rays hitting the grating at different times because the illumination of the grating is tilted $[9,10]$. By simply taking the product between the sweeping speed ( $\left.v_{\text {sweep }}\right)$ and the total sweeping time $\left(\tau_{\text {sweep }}\right)$, the length of the scanned line can be estimated to be $\lambda_{0} \cdot f / s$, which is the focused beam size corresponding to an individual monochromatic beam. Note that the sweeping speed is ultrafast. As an example, for a typical Ti:Sapphire laser where $\lambda_{0} \sim 800 \mathrm{~nm}$ and $\Delta \lambda \sim 10 \mathrm{~nm}$, if the back aperture of the objective is approximately filled by the spatially-chirped beam (i.e., with a NA $\sim 0.5$ and $f \sim \alpha \Omega$ ), we estimate that $v_{\text {sweep }} \sim 5 \times 10^{6} \mathrm{~m} / \mathrm{s}$.

In Figure 2.2, an intuitive ray/wave optics understanding of this pointsweeping process in line-scanning SSTF is illustrated. At the input focal plane of the objective, the input short pulse (pulse width $\sim \tau_{0}$ ) is first stretched into a long pulse (pulse width $\sim \tau_{0} \sqrt{1+\alpha^{2} \Omega^{2} / s^{2}}$ ) due to the separation of spectral components. This long pulse can be virtually decomposed into $\sqrt{1+\alpha^{2} \Omega^{2} / s^{2}}$ copies of spatiallyoverlapped but temporally-separated short pulses. Because of the ultrafast wavefront rotation caused by the spreading effect of the grating (see Eq. (2.6)), different copies of the short pulse will have different wavefront pointing directions, and therefore are focused to different lateral positions along the $x$-direction, forming a line that corresponds to $\sqrt{1+\alpha^{2} \Omega^{2} / s^{2}}$ number of point pixels in line-scanning mode.

For the application of SSTF to TPEF microscopy, a theoretical understanding of the axial confinement is important. Mathematically, this can be done by evaluating the total two-photon excited (TPE) signal on a perpendicular plane placed at position $z$ from the objective. Because the TPE signal is proportional to the fourth power of the focused field, by using the expression in Eq. (2.6), we have:

$$
\operatorname{TPE}(z)=\iiint_{\infty}\left|B_{2}(x, y, z, t)\right|^{4} d x d y d t=C_{2} \cdot \iint_{\infty}|h(x, y, z)|^{4} d x d y
$$




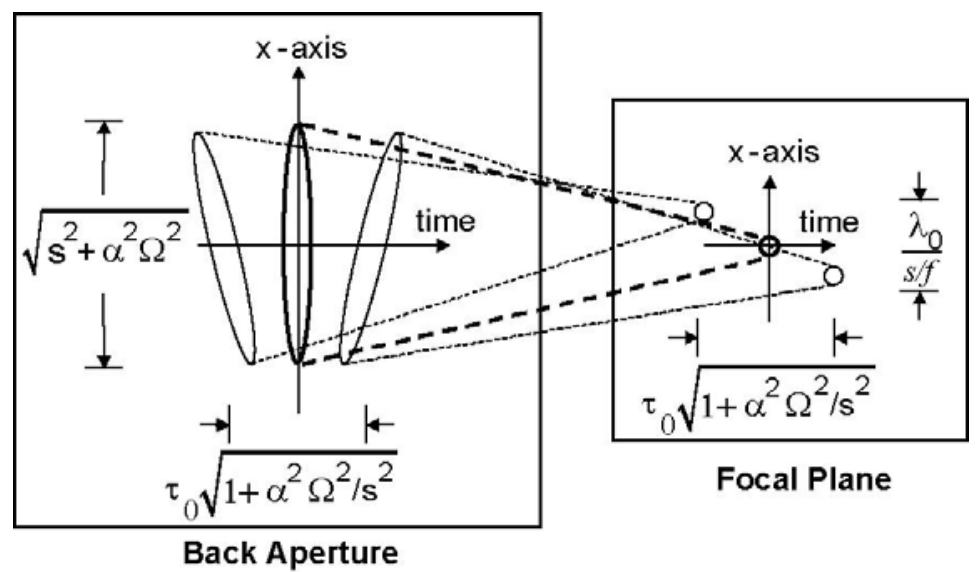

Figure 2.2: Intuitive understanding of SSTF in the time domain. The wavefront of the illumination is rapidly rotating, causing the focused spot to sweep along the $x$ direction and forming a line illumination with a width of $\lambda_{0} /(s / f)$, where $s / f$ represents the NA corresponding to an individual monochromatic beam. The total sweeping process takes $\tau_{0} \sqrt{1+\alpha^{2} \Omega^{2} / s^{2}}$ to complete. Note that the horizontal axis represents time and the vertical axis represents $x$. 
where $C_{2}$ is a constant independent of $z$. Equation (2.8) shows that the axial confinement is completely determined by the characteristics of the point-spread function of the objective lens $h(x, y, z)$. We thus conclude that it is the spatial profile of the spatially-chirped beam at the input focal plane of the objective that determines the axial confinement.

The above analysis focuses on the case of line-scanning operation of SSTF. For wide-field SSTF, a similar method based on the paraxial approximation can also be used to evaluate the performance of SSTF. In wide-field SSTF, the beam is only stretched along one dimension. As a result, a line-shaped beam is formed at the input focal plane of the objective. To establish a quantitative time-domain understanding for wide-field operation of SSTF, Eqs. (2.5)-(2.8) can be modified by simply eliminating the $y$-variable. Thus, for wide-field SSTF with a Gaussian spectrum, the point spread function in Eqs. (2.6)-(2.8) should be replaced by:

$$
h(x, z)=\int_{-\infty}^{\infty} P\left(x^{\prime}\right) e^{-\frac{x^{\prime 2}}{s^{2}+\alpha^{2} \Omega^{2}}} e^{i k_{0}\left(\frac{x x^{\prime}}{f}+z \frac{x^{\prime 2}}{2 f^{2}}\right)} d x^{\prime}
$$

We will discuss the implications of the axial confinement in wide-field SSTF in Section 2.4.

\subsection{Experiments}

\subsubsection{Experiment on temporal pulse-width evolution}

To demonstrate the temporal focusing effect, we employ the wide-field SSTF experimental setup shown in Figure 2.3. A ruled diffraction grating (groove density 1200 lines $/ \mathrm{mm})$ decomposes the beam from a mode-locked Ti:Sapphire laser $\left(\lambda_{0}=800\right.$ $\mathrm{nm}, \Delta \lambda=8 \mathrm{~nm}$ ) into its monochromatic components, which are then collimated using a spherical lens. Note that the geometric dispersion caused by the grating is automatically cancelled after collimation, and therefore the spread of beams is chirp 


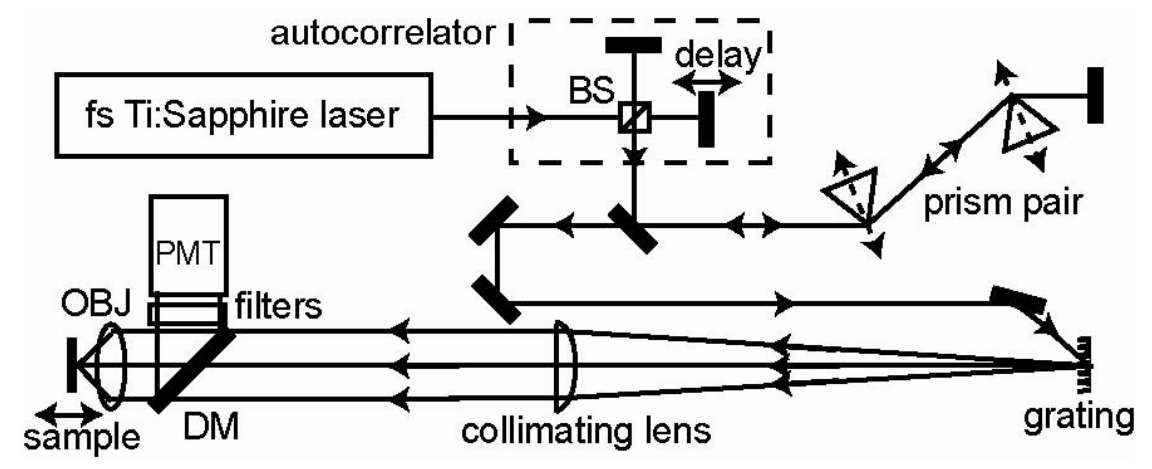

Figure 2.3: SSTF experimental setup. BS: beam splitter. DM: dichroic mirror. OBJ: objective lens. A mode-locked Ti:Sapphire laser (Tsunami, Spectra-Physics) serves as the excitation source. 
free in spectrum. The simultaneous spatial and temporal focusing effect is then realized by passing the beams through an objective lens. To collect the fluorescence signal, a dichroic mirror reflects the TPEF signal onto a PMT (Hamamatsu HC125-02) through filters that further remove the excitation light.

We measure the evolution of the temporal pulse width along the propagation direction in an interferometric second-order autocorrelator, which uses TPEF from a thin Rhodamine B sample as the nonlinear element [8]. The autocorrelation traces at the focal plane and far away from the focus are shown in Figure 2.4(a) and (b), respectively. At $275 \mu \mathrm{m}$ away from the focus (Figure 2.4(b)), a 1.64-ps pulse was measured and the characteristics of the interferometric autocorrelation trace were indicative of a highly chirped pulse. At the focal plane of the objective lens, however, a nearly chirp-free pulse is obtained, and the measured 84-fs pulse width (Figure 2.4(a)) is close to the original laser output. Figure 2.5 shows the measured temporal pulse width (solid squares) at various distances away from the focal plane by translating the thin Rhodamine B sample. Our measurements indicate that a value of 25 for the pulse width stretching factor (PWSF), which is defined as the ratio between the pulse width measured at the input focal plane of the objective lens and the pulse width measured at the focal point, could be achieved using the current setup. Note that in wide-field

SSTF, this PWSF is critical for suppressing the background TPEF signal and performing optical sectioning. Using the model described in the Section 2.2.1, a fitting curve for the pulse width (Eq. (4)) is plotted in Figure 2.5 (solid line). Good agreement between the calculated and measured data is obtained.

\subsubsection{Experiment on axial confinement}

In this section, we experimentally measure the axial confinement of wide-field 


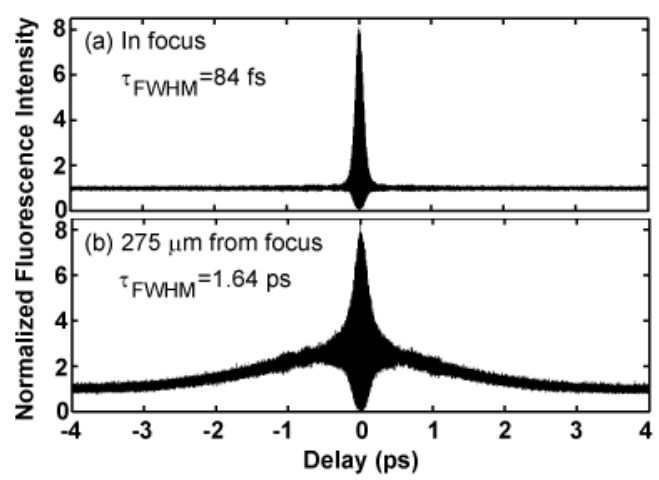

Figure 2.4: Autocorrelation traces of the measured pulse at different sample positions: (a) at the focal plane of the objective, (b) when moved $275 \mu \mathrm{m}$ away from focal plane. 


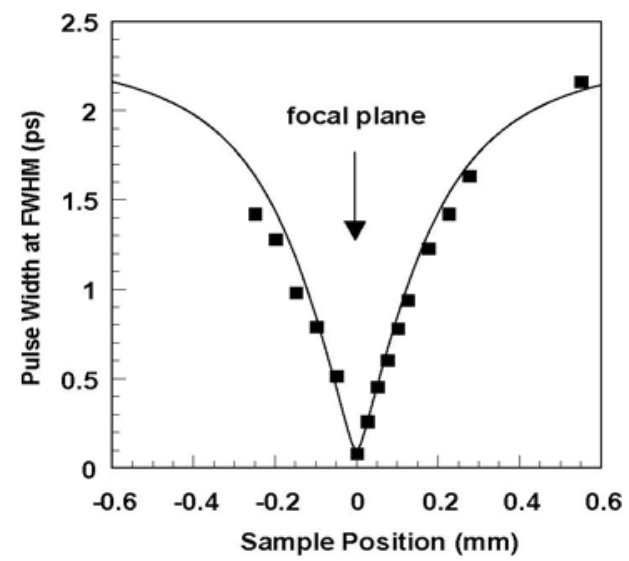

Figure 2.5: Measured (solid squares) and theoretical (line) pulse width as a function of sample position. The location of the focal plane of the objective lens is set to zero. 
SSTF as well as the peak TPEF signal counts. In this experiment (Figure 2.3), the center wavelength of the Ti:Sapphire laser is $775 \mathrm{~nm}$, the FWHM of the spectrum is $8.2 \mathrm{~nm}$, and the groove density of the grating is 1200 lines $/ \mathrm{mm}$. The objective used in this experiment has a NA of 0.45 . An aperture with radius $2.5 \mathrm{~mm}$ is placed in front of the objective back aperture in order to observe the finite back-aperture size effect easily. To vary the beam size, a set of collimating lenses with different focal lengths are used.

We define the axial confinement as the FWHM of the TPE $(z)$ traces (from Eq. (2.8)) obtained by scanning a thin Rhodamine B slide through the focal plane. The axial confinement and the TPEF counts generated from the peak position are measured for each focal length of the collimating lens. The results are plotted in Figure 2.6(a) and (b) (solid squares). Note that the size of the spatially chirped beam at the back aperture is linearly proportional to the focal length of the collimating lens. We show in this experiment that the axial confinement is determined by the beam size at the input focal plane of the objective. Also shown in Figure 2.6 are the values calculated using the time-domain theory (solid lines), i.e. Eq. (2.6) and Eq. (2.8) but with Eq. (2.9) as the point spread function. Note that in order to get the best fit, a sech-squared spectrum profile is used instead of a Gaussian spectrum in Eq. (2.9). The measured data agrees well with the theoretical analysis.

\subsection{Discussion on axial confinement enhancement}

It has been shown that the axial characteristics of SSTF with TPEF are determined by the input spatial profile. It is therefore straightforward to compare the focusing in the SSTF regime to that of regular imaging (Table 2.1). In wide-field SSTF, the beam is stretched only along one dimension to form a line-shape 
illumination at the input focal plane of the objective (Figure 2.1). This is equivalent to the beam profile in a regular line-scanning setup, and thus the axial confinement of wide-field SSTF and a conventional line-scanning system are the same. In linescanning SSTF, the beam has a circular cross-section at the input focal plane of the objective, and therefore its axial confinement will be equivalent to that of a regular point-scanning system [11]. Thus, SSTF provides an improved axial confinement when compared to conventional systems in the same imaging configuration (wide field or line scanning). Intuitively, the temporal focusing in SSTF replaces the focusing of one spatial dimension in a conventional imaging system. The best axial confinement achieved by SSTF (i.e., line scanning) cannot surpass that of a regular point-scanning TPEF system.

In practice, the axial resolution and the TPEF signal of SSTF microscopy are important design issues. Based on Eq. (2.8), it is apparent that these parameters for SSTF should follow exactly that of the regular TPEF microscopy, i.e. achieving higher resolution by further expanding the spatially-chirped beam at the input focal plane of the objective, although at the cost of less signal generated at the sample. These concepts are expressed in the experimental data found in Section 2.3.2 for wide-field SSTF (Figure 2.6). When the spatially-chirped beam is large (corresponding to a collimating lens with a long focal length), the FWHM of the axial confinement approaches that of a regular line-scanning system (Figure 2.6(a)). The generated TPEF signal, however, is low. On the other hand, when the size of the spatiallychirped beam is reduced, the FWHM of the axial confinement increases monotonically while the TPEF at the focal plane first increases, reaches a maximum, and then decreases. The initial increase is due to the improved power transmission of the monochromatic beams, which simultaneously increases the excitation power and shortens the temporal pulse width. The latter decrease is due to the lack of spatial 

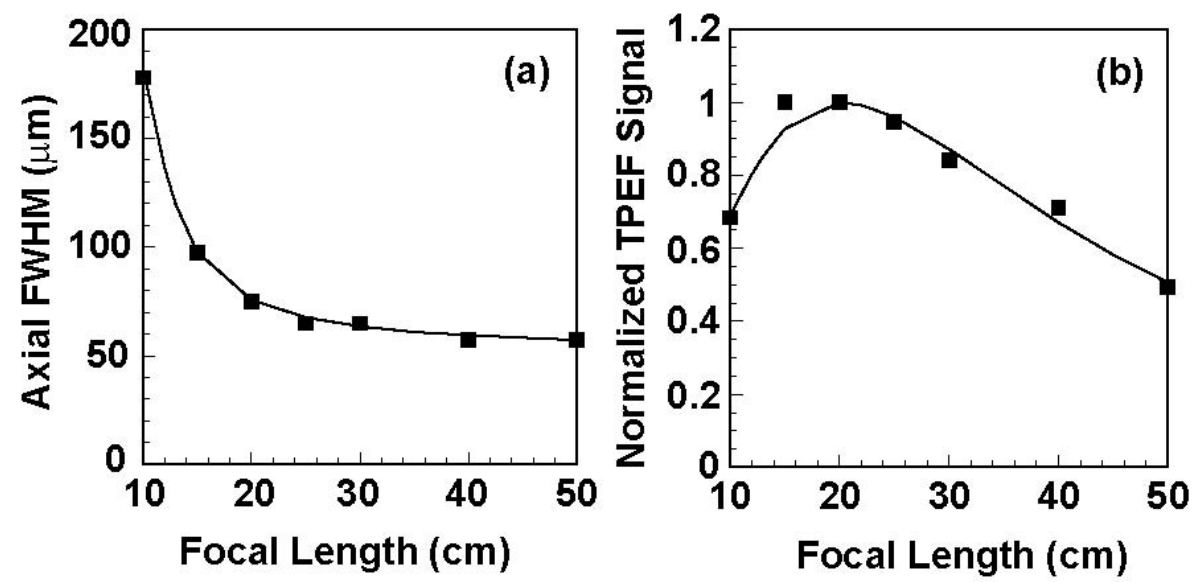

Figure 2.6: Experimental measurement (squares) and theory (lines) for wide-field SSTF (a) FWHM of the axial confinement function $\operatorname{TPE}(z)$ and (b) normalized signal power of the TPEF generated by a thin fluorescence film placed at the focal plane. 
Table 2.1: Comparison between different conventional imaging systems with those of SSTF. For conventional imaging, Gaussian beam propagation in the paraxial limit is used, where $z_{\mathrm{R}}$ represents the Rayleigh range of the beam. In SSTF imaging, $z_{\mathrm{R}}$ represents the Rayleigh range of the spatially-chirped beam as defined in Eq. (2.3). In both cases, $\Delta \mathrm{z}$ refers to the displacement from the focus.

\begin{tabular}{|c|c|c|c|}
\hline & Point scan & Line scanning & Wide field \\
\hline $\begin{array}{c}\text { Conventional } \\
\text { Imaging }\end{array}$ & $\sim\left[1+\left(\frac{\Delta z}{z_{R}}\right)^{2}\right]^{-1}$ & $\sim\left[1+\left(\frac{\Delta z}{z_{R}}\right)^{2}\right]^{-\frac{1}{2}}$ & $\mathrm{~N} / \mathrm{A}$ \\
\hline $\begin{array}{c}\text { SSTF } \\
\text { Imaging }\end{array}$ & $\mathrm{N} / \mathrm{A}$ & $\sim\left[1+\left(\frac{\Delta z}{z_{R}}\right)^{2}\right]^{-1}$ & {$\left[1+\left(\frac{\Delta z}{z_{R}}\right)^{2}\right]^{-\frac{1}{2}}$} \\
\hline
\end{tabular}


focusing when the back aperture is underfilled. The same behavior is found with regular TPEF microscopy, indicating once again that it is the shape of the spatially chirped beam at the input focal plane of the objective that determines the axial confinement for SSTF.

In the time-domain understanding of SSTF, as illustrated by Figure 2.2, the total nonlinear signal in the focal volume is the sum of the contribution of many short pulses, which are obtained after virtually decomposing the stretched long pulse into many short pulses delayed in time. We can make two observations at the output focal plane of the objective. First, there is no spatial overlapping of the focused spots since different short pulses are focused to different lateral positions. Second, there is no temporal overlapping of the pulse arrival time simply because of the long pulse decomposition. It is thus clear that SSTF is similar to the temporally decorrelated multifocal multiphoton imaging technique $[12,13]$, but implemented in a continuous fashion and without the need to generate multiple beamlets.

The spatial and temporal trade-off in SSTF leads to a number of advantages. One of them is scanningless wide-field imaging [9]. Because the entire sample is illuminated, wide-field SSTF allows more excitation peak power to be directed onto the sample. In contrast to conventional wide-field imaging, wide-field SSTF provides optical sectioning. Imaging of biological tissues has been performed with wide-field SSTF [9], but the image acquisition time was long ( $\sim 30$ seconds). The use of a regenerative amplifier or a specially engineered dye with a greater two-photon crosssection could substantially decrease the image acquisition time, providing the possibility of the ultimate time resolution of one pulse per image. 


\subsection{Conclusion}

Simultaneous spatial and temporal focusing of femtosecond pulses improves the axial confinement in wide-field and line-scanning imaging systems. We have reviewed analytical expressions that describe the evolution of the pulse width within a TPEF sample. It is found that the axial confinement of SSTF nonlinear microscopy is determined by the spatial profile at the input focal plane of the objective. Thus, SSTF provides an improved axial confinement when compared to conventional wide-field and line-scanning systems, but the best axial confinement achieved by SSTF is the same as a regular point-scanning system. Because the temporal focusing in SSTF essentially replaces the focusing of one spatial dimension in a conventional system, this trade-off between spatial and temporal focusing can provide significant advantages in applications such as high-speed imaging and remote axial scanning in an endoscopic fiber probe.

\section{Acknowledgments}

The authors would like to thank Dr. W. Zipfel for providing the large mode area fiber and the laser facility in the early stages of the experiment. This research was made possible by Grant Number 1R21CA115472-01 from the National Cancer Institute (NCI), National Institutes of Health. 


\section{REFERENCES}

1. W. Denk, J. H. Strickler, and W. W. Webb, "2-Photon Laser Scanning Fluorescence Microscopy," Science 248, 73-76 (1990).

2. W. R. Zipfel, R. M. Williams, and W. W. Webb, "Nonlinear magic: multiphoton microscopy in the biosciences," Nature Biotechnology 21, 1368-1376 (2003).

3. J. P. Ying, F. Liu, and R. R. Alfano, "Effect of scattering on nonlinear optical scanning microscopy imaging of highly scattering media," Applied Optics 39, 509-514 (2000).

4. J. Goodman, Introduction to Fourier Optics (Roberts \& Company, Englewood, Colorado, 2005).

5. O. E. Martinez, "Grating and prism compressors in the case of finite beam size," J. Opt. Soc. Am. B 3, 929-934 (1986).

6. M. E. Durst, G. Zhu, and C. Xu, "Simultaneous spatial and temporal focusing for axial scanning," Optics Express 14, 12243-12254 (2006).

7. O. E. Martinez, "3000 Times Grating Compressor with Positive GroupVelocity Dispersion - Application to Fiber Compensation in 1.3-1.6 Mu-M Region," IEEE Journal of Quantum Electronics 23, 59-64 (1987).

8. M. Gu, Advanced Optical Imaging Theory (Springer, New York, 2000).

9. D. Oron, E. Tal, and Y. Silberberg, "Scanningless depth-resolved microscopy," Optics Express 13, 1468-1476 (2005).

10. D. Oron, and Y. Silberberg, "Harmonic generation with temporally focused ultrashort pulses," Journal of the Optical Society of America B-Optical Physics 22, 2660-2663 (2005).

11. E. Tal, D. Oron, and Y. Silberberg, "Improved depth resolution in video-rate 
line-scanning multiphoton microscopy using temporal focusing," Optics Letters 30, 1686-1688 (2005).

12. D. N. Fittinghoff, P. W. Wiseman, and J. A. Squier, "Widefield multiphoton and temporally decorrelated multifocal multiphoton microscopy," Optics Express 7, 273-279 (2000).

13. V. Andresen, A. Egner, and S. W. Hell, "Time-multiplexed multifocal multiphoton microscope," Optics Letters 26, 75-77 (2001). 


\title{
CHAPTER 3
}

\section{SIMULTANEOUS SPATIAL AND TEMPORAL FOCUSING FOR AXIAL $\mathrm{SCANNING}^{4}$}

\begin{abstract}
We show theoretically and experimentally that simultaneous spatial and temporal focusing can scan the temporal focal plane axially by adjusting the group delay dispersion in the excitation beam path. When the group delay dispersion is small, the pulse width at the temporal focal plane is transform-limited, and the amount of shift depends linearly upon the dispersion. By adding a meter of large mode area fiber into the system, we demonstrate this axial scanning capability in a fiber delivery configuration. Because a transform-limited pulse width is automatically recovered at the temporal focal plane, simultaneous spatial and temporal focusing negates the need for any dispersion pre-compensation, further facilitating its integration into a fiber delivery system. A highly promising application for simultaneous spatial and temporal focusing is an axial scanning multiphoton fluorescence fiber probe without any moving parts at the distal end and without dispersion pre-compensation.
\end{abstract}

\subsection{Introduction}

The advantage of multiphoton microscopy (MPM) for imaging is its ability to perform optical sectioning in highly scattering samples [1]. The application of MPM to a flexible fiber endoscope has been slow due to the difficulty in miniaturizing mechanical scanning components [2]. Transverse scanning has been performed on the proximal end of a fiber bundle in both one-photon confocal [3-5] and two-photon microscopy [6], but focusing and axial scanning invariably requires mechanical

\footnotetext{
${ }^{4}$ The content of this chapter has been reproduced from Optics Express 14, 12243-12254 (2006).
} 
motion on the distal end of an endoscope $[7,8]$. As seen in recent reports of multiphoton endoscopes $[2,9]$ and miniature microscopes $[10,11]$, these mechanical scanning devices are the main limitation in the fabrication of a flexible and compact endoscope, characteristics that are crucial to make multiphoton imaging compatible with existing endoscopes.

A few axial scanning methods have been applied to a two-photon excited fluorescence (TPEF) fiber probe. In the simplest case, axial scanning can be performed by physically moving the sample [12] or a lens at the distal end of the fiber [13]. A hydraulic system has been employed to adjust the suction applied to a tissue specimen, thus changing the focal plane by varying the distance between the fiber and the objective [14] or between the objective and the sample [4]. Also, a liquid lens can be used at the distal end of the fiber, where adjusting the voltage across the interface of two liquids changes the radius of curvature of the meniscus, thus varying the focal length [15], [16]. A spatial light modulator (SLM) has also been applied to provide wavefront correction and axial focal shift $[17,18]$. A disadvantage of these techniques is that they operate at the distal end of the fiber. If the fiber probe is a gradient refractive index (GRIN) lens, however, adjusting the coupling into the proximal end scans the focal plane because the GRIN fiber acts as a thick lens $[2,19]$. There are many ways to adjust the coupling into the GRIN lens, including piezo-stages [19], micromotors [20], and an adaptive mirror [21]. However, the rigid nature of the GRIN lens makes it best suited for a rigid endoscope or a miniature microscope. When a GRIN lens is affixed to the end of a flexible fiber endoscope, though, these coupling components must be added to the distal end of the fiber.

Simultaneous spatial and temporal focusing (SSTF), a MPM imaging technique, improves the axial resolution and reduces the background by broadening the pulse width everywhere but at the focal volume $[22,23]$. SSTF works by spatially 
separating the frequencies of a short pulse with a grating, collimating these monochromatic beams with a cylindrical lens, and recombining them with an objective lens (Figure 3.1). A temporal focus occurs because the different frequency components only overlap within the focal region of the objective lens. Thus, the pulse width is shortest only at the focal plane, and, as described in [22], the pulse width is transform-limited.

It was mentioned in a previous paper [22] that the presence of group delay dispersion (GDD) in an SSTF system increases the optical path length of the system. Therefore, the plane of the temporal focus will be displaced along the optical axis from the geometrical focus of the objective lens, yielding z-scanning as a function of dispersion [18]. In this paper, the authors theoretically and experimentally demonstrate that changing the GDD of the system results in axial scanning of the temporal focal plane. In Section 3.2, we derive the fluorescence signal as a function of dispersion and position $z$ along the optical axis using Fresnel diffraction. In Section 3.3, we demonstrate experimentally the dependence of focal plane position on GDD, and the remote axial scanning capability in a fiber delivery configuration. In Section 3.4, we discuss the practical requirements for applying this technique to both a multiphoton microscope and a remote axial scanning, flexible fiber probe.

\subsection{Theoretical analysis of SSTF with GDD}

Axial scanning in an SSTF system by tuning the amount of GDD in the beam path can be intuitively understood by following the wavefront of the pulse as it propagates through the system. After diffracting off the grating and being collimated by a lens (Figure 3.1), the beam becomes a spread of individual monochromatic beams, creating a one-to-one correspondence between the spectral frequency $\omega$ and 


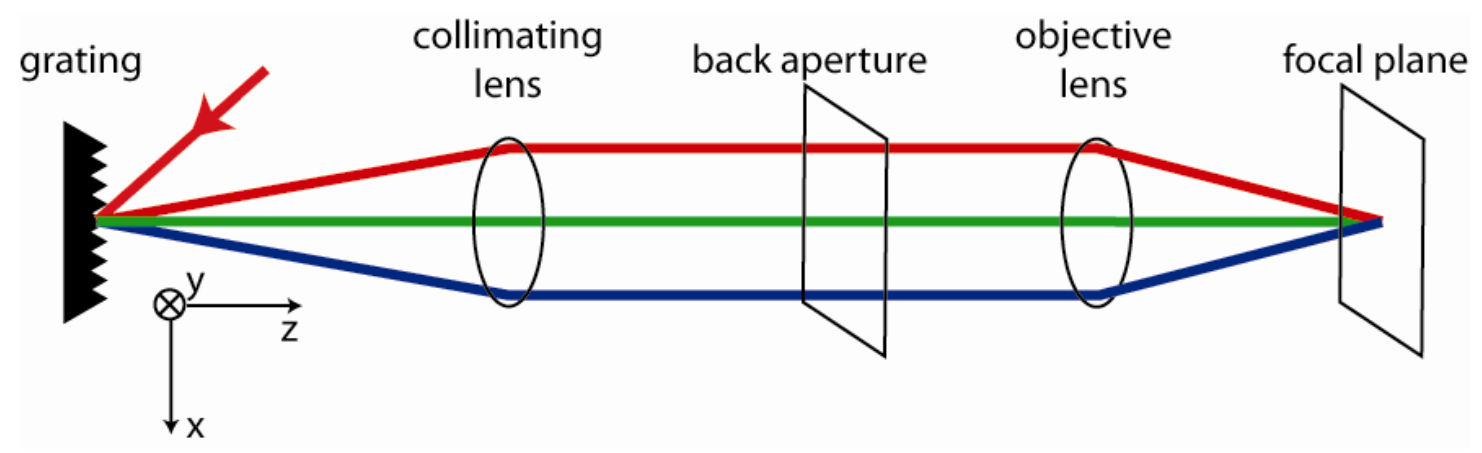

Figure 3.1: A typical SSTF setup. 
the transverse position $x$. Thus, any curvature in the spectral phase will directly translate into a curvature of the wavefront in space. The wavefront is flat for a chirpfree pulse, but if there is any second-order chirp, this quadratic spectral phase will result in a quadratically-curved wavefront. This is equivalent to adding an extra lens in space to the system. Adjusting the amount of GDD is analogous to changing the curvature of the lens, thus shifting the position of the focal plane.

The theoretical understanding of axial scanning with SSTF follows closely the derivation in Chapter 2, except that the incident pulse is now chirped. For one individual frequency $\omega$, the beam amplitude at the input focal plane of the objective lens is:

$$
A_{1}(x, \omega)=A_{0} e^{-\frac{\omega^{2}}{\Omega^{2}}} e^{-\frac{(x-\alpha \omega)^{2}}{s^{2}}} e^{i \beta \omega^{2}}
$$

where $A_{0}$ is a normalization constant, $\sqrt{2 \ln 2} \cdot \Omega$ is the full-width half maximum (FWHM) of the frequency spectrum of the pulse, $\sqrt{2 \ln 2} \cdot s$ is the FWHM of each monochromatic beam in space, $\alpha$ is a constant proportional to the groove density of the grating and the focal length of the collimating lens [24], $\alpha \omega$ is the linear displacement of the monochromatic beam of frequency $\omega$, and $2 \beta$ is the GDD. The imaginary term in Eq. (3.1) represents the second-order chirp (quadratic spectral phase) of the input pulse.

In order to analytically describe the field at the output, we follow the paraxial approximation used in Ref. [25] to propagate the beam to the focal volume (for detailed calculations, see Appendix B). The spatially-chirped beam, $A_{1}(x, \omega)$, is incident upon the input focal plane of the objective lens. First, the Fresnel diffraction formula is applied to $A_{1}$ to propagate the beam to the objective lens. After adding the quadratic phase due to the objective lens, the Fresnel diffraction formula is used again to propagate it a distance $z$ toward the focal plane. Fourier transforming back into the 
time domain expresses the field in the focal volume. To simplify calculations, we assume that the wavevector $k$ for each frequency is approximately $k_{0}$, the wavevector of the center wavelength of the pulse, which is valid for pulse widths on the order of 100 fs. Integrating the squared intensity $I$ over time and the transverse direction $x$ yields the total two-photon excitation (TPE) signal at an axial plane at position $z$ :

$$
\operatorname{TPE}(z)=\int_{-\infty}^{\infty} \int_{-\infty}^{\infty} I^{2}(x, z, t) d x d t=\frac{C}{\left[\left(1+\beta \Omega^{2} \frac{z-f}{z_{M}}\right)^{2}+\left(\frac{f-z+\beta \Omega^{2} z_{R}}{z_{R}}\right)^{2}\right]^{\frac{1}{2}}}
$$

where $z_{M}=2 f^{2} / k_{0} s^{2}, z_{R}=\left(2 f^{2} / k_{0}\right) /\left(s^{2}+\alpha^{2} \Omega^{2}\right), f$ is the focal length of the objective lens, $C$ is a constant, and $z_{M}$ is the Rayleigh length of the focused monochromatic beam. Intuitively, $\left(s^{2}+\alpha^{2} \Omega^{2}\right)^{\frac{1}{2}}$ is the width of the spatially-chirped beam before the objective lens. Thus, $z_{R}$ is the Rayleigh length of the focused spatially-chirped beam.

If $\beta \Omega^{2}$ is small, then Eq. (3.2) can be approximated as:

$$
\operatorname{TPE}(z) \approx \frac{C}{\left[1+\left(\frac{f-z+\beta \Omega^{2} z_{R}}{z_{R}}\right)^{2}\right]^{\frac{1}{2}}}
$$

As seen in Eq. (3.3), the axial response $T P E(\mathrm{z})$ has a peak of constant value $C$, i.e., independent of GDD, at the temporal focal plane position $z=f+\beta \Omega^{2} z_{R}$. The FWHM of $\operatorname{TPE}(\mathrm{z})\left(2 \sqrt{3} \cdot z_{R}\right)$ defines the axial resolution. Eq. (3.3) also shows that the shift of the focal plane position has a linear dependence on GDD when $\beta \Omega^{2}$ is small:

$$
\Delta z_{\text {shift }}=\beta \Omega^{2} z_{R}
$$

The pulse width at the temporal focus can also be obtained (see Appendix B) as:

$$
\tau=\tau_{0} \cdot\left[1+\left(\beta \Omega^{2}\right)^{2} \frac{z_{R}}{z_{M}}\right]^{\frac{1}{2}}
$$

A small $z_{R}$ is needed to obtain high axial resolution, but to achieve the desired 
axial scan range $\left(\Delta z_{\text {shift }}\right)$, the dispersion value $\beta$ must be increased when $z_{R}$ decreases (Eq. (3.4)). There will be a limit, however, to the amount of shift that GDD can produce before the assumption that $\beta \Omega^{2}$ is small is no longer valid. Thus, the exact expression of $T P E(z)$ in Eq. (3.2) must be used to predict the behavior of $\operatorname{TPE}(z)$. In this regime, the maximum value of $\operatorname{TPE}(z)$ is no longer a constant and decreases with increasing GDD. Intuitively, the monochromatic beams fail to overlap completely as the temporal focal plane is shifted a large distance away from the geometric focus, therefore broadening the pulse as well as increasing the lateral spot size (Figure 3.2).

We define the onset of broadening as when the maximum value of $\operatorname{TPE}(z)$ in Eq. (3.2) is reduced to half its value when GDD is zero. Using the approximate peak position as $z=f+\beta \Omega^{2} z_{R}$ and setting Eq. (3.2) equal to $C / 2$, we determine the maximum amount of $\beta \Omega^{2}$ possible without broadening to be:

$$
\left.\beta \Omega^{2}\right|_{\max }=\left(\frac{z_{M}}{z_{R}}\right)^{\frac{1}{2}}=\left(\frac{s^{2}+\alpha^{2} \Omega^{2}}{s^{2}}\right)^{\frac{1}{2}} \approx \frac{\alpha \Omega}{s}
$$

Substituting Eq. (3.6) into our expression for the pulse width (Eq. (3.5)), the pulse width can be expressed as:

$$
\tau=\tau_{0} \cdot\left[1+\left(\frac{\beta \Omega^{2}}{\left.\beta \Omega^{2}\right|_{\max }}\right)^{2}\right]^{\frac{1}{2}}
$$

As seen in Eq. (3.7), the onset of broadening has been defined as when the pulse width at the temporal focal plane is $\tau=\sqrt{2} \tau_{0}$. Combining Eq. (3.4) and Eq. (3.6), the maximum shift allowed by SSTF is:

$$
\Delta z_{\max }=\left.\beta \Omega^{2}\right|_{\max } \cdot z_{R}=\left(z_{M} z_{R}\right)^{\frac{1}{2}} \approx \frac{\alpha \Omega}{S} z_{R}
$$

Eq. (3.8) shows that the number of resolvable z-sections $\left(\sim \Delta z_{\max } / z_{R}\right)$ is simply the ratio between the widths of the spatially chirped beam $(\sim \alpha \Omega)$ and the monochromatic beam $(s)$ at the input focal plane of the objective lens. In practice, the 


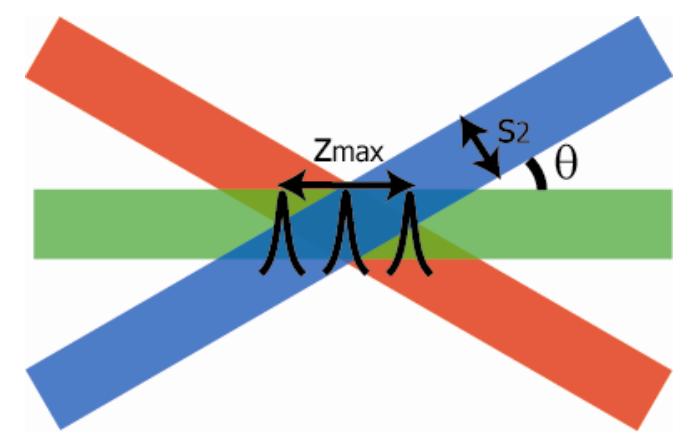

Figure 3.2: A ray-tracing representation of the focal volume shows intuitively the fundamental scanning limit, where $s_{2}$ is the lateral spot size of a monochromatic beam and $z_{\max }$ is the length of the geometrical overlap. 
axial resolution (proportional to $\left.z_{R}\right)$ and maximum shift $\left(\Delta z_{\max }\right)$ can be scaled for a particular application. For example, a higher z-resolution (smaller $z_{R}$ ) will be possible if the maximum scanning range $\left(\Delta z_{\max }\right)$ is sacrificed.

\subsection{Axial scanning experiment}

The experimental SSTF setup is shown in Figure 3.3. We use a mode-locked Ti:Sapphire laser (Spectra-Physics Tsunami) centered at $775 \mathrm{~nm}$ with 60-100 fs pulses (assuming a sech shape) at an $80 \mathrm{MHz}$ repetition rate. To measure the pulse width at the sample, the setup has a built-in interferometric second-order autocorrelator which uses TPEF from a thin Rhodamine film ( $\sim 1 \mu \mathrm{m}$ thick) on a \#1 cover glass as the nonlinear element. A prism pair changes the GDD in the system by adjusting the amount of glass in the beam path. A beam shrinker composed of two plano-convex lenses controls the monochromatic beam size $s$. A ruled diffraction grating with 1200 lines per mm separates the beam into its monochromatic components, which are then collimated by a spherical lens. The objective lens is a 10x/0.45 NA Zeiss PlanApochromat. To collect the fluorescence signal, a dichroic mirror reflects the TPEF signal onto a PMT (Hamamatsu HC125-02) through filters that further remove the excitation light.

In order to observe shifting of the temporal focus, we measure $\operatorname{TPE}(z)$ traces by scanning the Rhodamine B film sample on a translation stage. We perform these axial scans at different GDD values and record the peak position, i.e., the position of the temporal focal plane. Figure 3.4(a) shows the temporal focal plane position as a function of GDD. A linear relationship is observed between GDD and the displacement of the focal plane, in agreement with theory (Eq. (3.4)). We are able to shift the temporal focus by more than $130 \mu \mathrm{m}$, limited by the thickness of the prisms. 


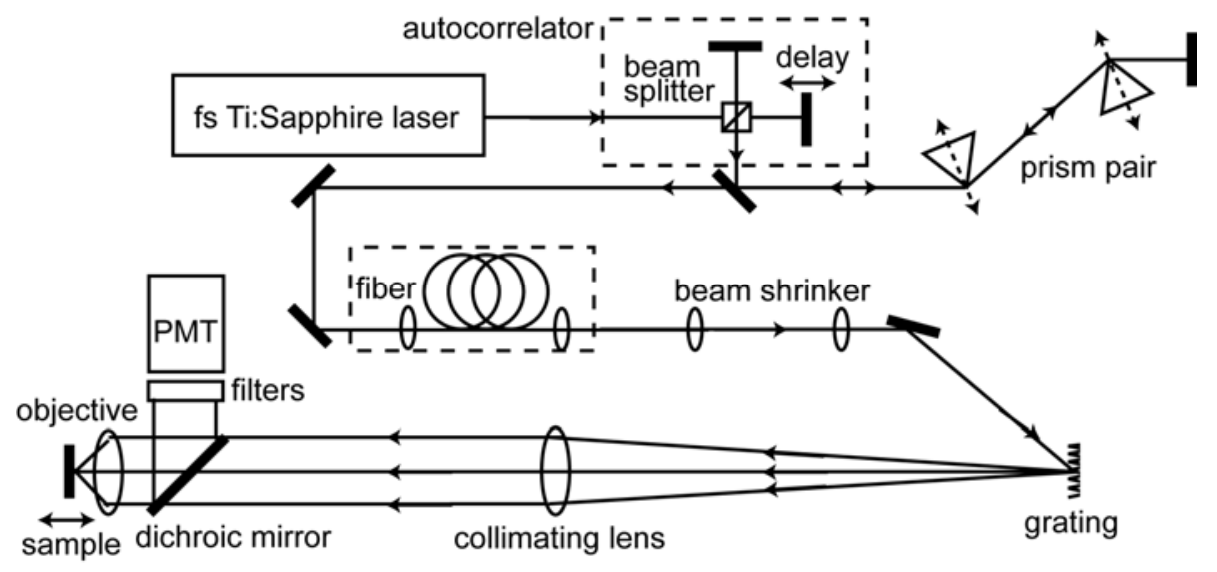

Figure 3.3: SSTF setup composed of a mode-locked Ti:Sapphire laser tuned to 775 nm, a 1200 lines/mm grating, a 4x beam shrinker, a Zeiss Apochromat 10x/0.45 objective lens, and, for fiber delivery experiments, one meter of large mode area fiber coupled by two 0.1 NA objective lenses. 
(a)

(b)

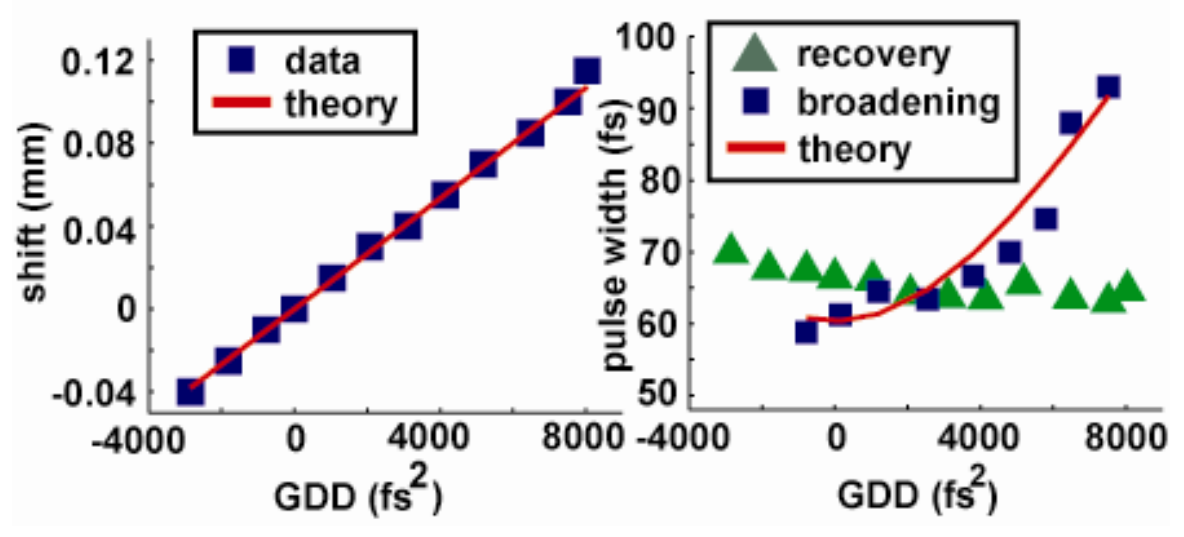

Figure 3.4: (a) The shift of the temporal focal plane position is plotted versus GDD varied by adjusting the prism pair. The squares represent peak positions of axial traces taken for various prism positions. The solid line is the expected linear relationship. In (b), the data points represent the pulse width at the sample for various GDD values.

The triangles demonstrate the recovery of the pulse width for a 1200 lines per millimeter grating and a $4 \mathrm{x}$ beam shrinker. The squares show that the pulse broadens with added GDD when the grating is changed to 600 lines per millimeter and the beam shrinker is reduced to $2 \mathrm{x}$. The solid red line is the predicted pulse width for the broadening case. 
Despite the addition of extra dispersion, a nearly transform-limited pulse width is recovered for each of these data points (triangles in Figure 3.4(b)). The measured ratio $\alpha \Omega / s$ in our experiment is $\sim 22$. For a 65 fs pulse width (bandwidth $\Omega$ of $3.8 \times 10^{13}$ $\mathrm{Hz}$ ), the recovered pulses are expected to broaden near a GDD value of $3.0 \times 10^{4} \mathrm{fs}^{2}$ (Eq. (3.6)), corresponding to a maximum shift of $0.5 \mathrm{~mm}$.

In order to demonstrate the broadening of the pulse when the shifting of the focal plane becomes large, we measure the pulse width at the sample using a different set of experimental parameters. Using the same GDD tuning range, we reduce the ratio $\alpha \Omega / s$ to 5 by using a grating with only 600 lines per millimeter, a $2 x$ beam shrinker, and a slightly shorter initial pulse width of 60 fs (Figure 3.4(b)). Pulse broadening now occurs at a GDD value of $6.7 \times 10^{3} \mathrm{fs}^{2}$ yielding a shift of $350 \mu \mathrm{m}$. As seen in Figure 3.4(b), our experimental results agree reasonably well with the theoretical prediction (Eqs. (3.6) and (3.7)).

The main advantage of shifting the focal plane by tuning the GDD in SSTF is the ability to perform remote axial scanning in a fiber delivery system, i.e., the scanning mechanism is at the proximal end of the fiber. To demonstrate the feasibility of such a remote axial scanning capability in a fiber delivery system, we insert $\sim 1 \mathrm{~m}$ of large mode area fiber (core diameter $25 \mu \mathrm{m}$, Crystal Fibre) between the laser and the grating (Figure 3.3). This fiber was chosen due to its low nonlinearity even at high input power [26]. Using a \#1 cover glass coated on both sides with a thin film of Rhodamine B, we scan the sample stage through the focal plane at a constant GDD value (solid line in Figure 3.5). Then, with the sample fixed, we scan through both the front and back films of the slide by adjusting the dispersion in the system (dots in Figure 3.5). An extra prism pair is added to the setup in Figure 3.5 to increase the GDD tuning range. Thus, we were able to shift the temporal focal plane by 200 microns when $z_{R}$ is 11.5 microns, revealing both the front and back films of the cover 


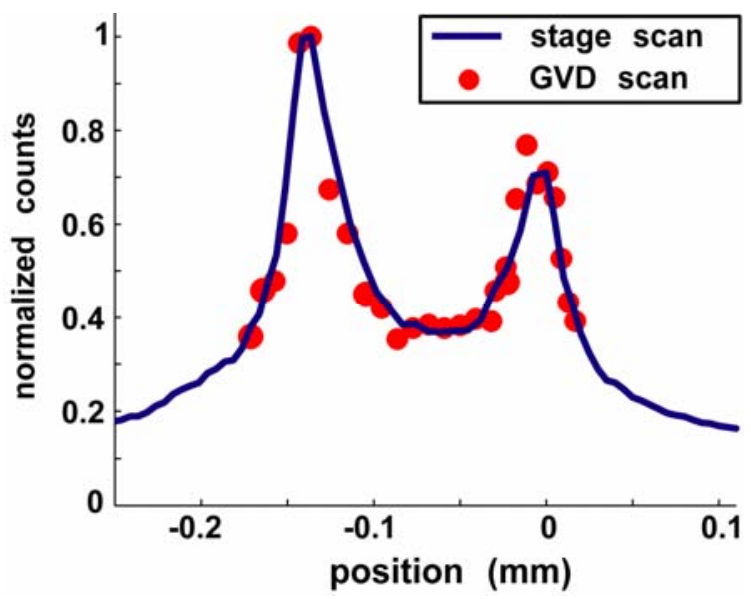

Figure 3.5: $\quad$ Scanning of a \#1 cover glass coated on both sides with a thin

Rhodamine B film. The blue curve is an axial trace obtained by scanning the sample with a translation stage. The red circles represent data taken of the axial scan by tuning the GDD at a fixed sample position. 
glass. The axial scans obtained by translating the sample and by adjusting the dispersion are nearly identical, demonstrating the feasibility of remote scanning of the focal plane by adjusting the GDD. We note that the $z$ values corresponding to points within the cover glass have been scaled by 1.5 to take into account refraction due to the air-glass interface. The different peak values from the front and back surfaces of the slide are due to a combination of the absorption of the TPEF signal of the back film by the front film and the distinct thicknesses of the two films.

In addition, the ability of SSTF to recover a transform-limited pulse width despite the presence of large chirp negates the need for any dispersion precompensation in a fiber delivery system (typically by using a prism pair), improving the practicality of the system. For example, when an $87 \mathrm{fs}$ pulse is launched into the large mode area fiber, the pulse broadens to $1.27 \mathrm{ps}$ at the output of the fiber due to its large dispersion (Figure 3.6(a)). At the temporal focus, a nearly chirp-free 99 fs pulse width is recovered (Figure 3.6(b)) without any dispersion pre-compensation. The 10\% pulse broadening is predicted by our theory using the calculated GDD value of the fiber as $3.5 \times 10^{4} \mathrm{fs}^{2}$. In essence, the SSTF setup acts as a post-dispersion compensation device, automatically removing the chirp of the pulse at the temporal focus. Although fibers have been engineered to exhibit both low nonlinearities and zero dispersion within a specific wavelength range [27], this technique allows short pulses to be delivered to the sample over a broad spectral range without any precompensation of dispersion.

\subsection{Discussion}

In an SSTF axial scanning setup, the $z$-resolution (proportional to $z_{R}$ ), lateral spot size (inversely proportional to $s$ ), and maximum shift $\left(\Delta z_{\max }\right)$ can be scaled for a 

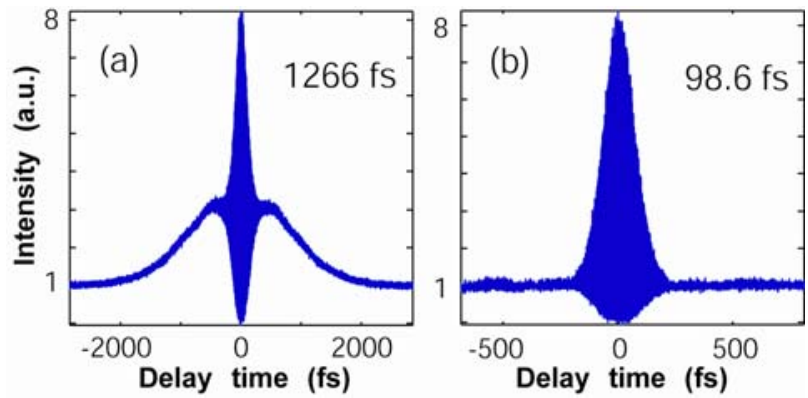

Figure 3.6: Autocorrelation traces for a system without dispersion compensation where the pulse has propagated through one meter of large mode area fiber. The laser has a center wavelength of $775 \mathrm{~nm}$ and a spectral width of $8 \mathrm{~nm}$. In plot (a), the pulse is at the output of the large mode area fiber, and in (b), at the temporal focal plane of the SSTF setup. 
particular application. For example, a larger lateral spot size (i.e., smaller $s$ ) will provide a larger scan range for a given z-resolution. As an example, a 10 micron $z$ resolution ( $z_{R}=3$ microns) with a spot size of 150 microns can scan 435 microns before the pulse begins to broaden. Alternatively, an improved $z$-resolution is possible if we sacrifice the maximum scanning range. Tal et al. achieved a 1.5 micron $z$-resolution with temporal focusing for video-rate imaging [28]. For this $z$-resolution with a 35 micron spot size, the axial scanning range would be 40 microns. Experimentally, the scaling of the $z$-resolution, lateral spot size, and the maximum scanning range is readily achieved by choosing the right combinations of the focal lengths for the objective and collimating lenses, the groove density of the grating, and the input beam size.

We neglected the vertical dimension in our derivation in Section 3.2 by assuming the beam shape is a thin line in the $x$-direction at the input focal plane of the objective lens. Here, the width in the $x$-direction is the spreading of the monochromatic components due to the grating, and the height in the $y$-direction is the monochromatic beam size $s$. Because $s$ is small, the $y$-component of the beam will not focus tightly. Such a focusing configuration is wide-field SSTF because it creates a large spot at the focal plane. In the SSTF line-scanning case, the beam has a circular cross-section at the input focal plane of the objective lens, so that the height ( $y$ dimension) of the monochromatic components will tightly focus to form a line at the geometrical focus. Because TPEF is inversely proportional to both the illuminated area and pulse width, the line scanning case results in two peaks when the geometric and temporal foci do not overlap. To demonstrate this effect experimentally, we expand the $y$-component of the beam with a cylindrical lens pair. As seen in Figure 3.7, changing the dispersion moves the temporal focus, but a stationary peak from the 

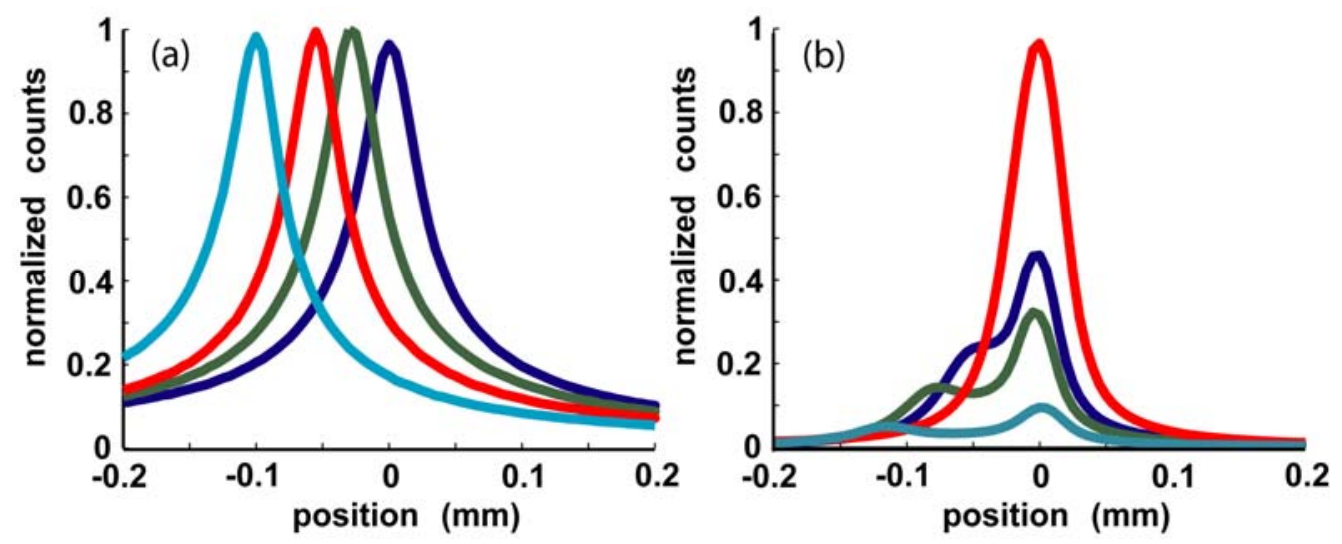

Figure 3.7: Axial scan data obtained by GDD tuning for (a) wide-field and (b) linescanning SSTF. 
geometric focus remains. Thus, axial scanning of the temporal focal plane by tuning the GDD works best in the wide-field SSTF configuration.

As opposed to conventional imaging, wide-field imaging with SSTF provides optical sectioning as well as axial scanning using GDD tuning. In practice, the widefield image is captured on a CCD camera [22]. As the focal plane scans, the camera must be moved back into focus for each temporal focus position. For in vivo endoscopic imaging, however, having a moving camera at the distal end of the fiber would be difficult. We believe that the demonstrated remote axial scanning technique is best suited in a passive, flexible fiber probe for multiphoton excitation of $z$-resolved fluorescence or harmonics, bridging the gap between imaging optical endoscopes and non-imaging fiber optic probes. It has all the desirable attributes of a passive fiber probe but with added $z$-sectioning capability over an axial scan range of hundreds of microns. As shown previously in tissue spectroscopy and imaging [29-31], the addition of $z$-sectioning to a conventional fiber probe will undoubtedly improve its capability in medical detection and diagnostics.

To create such a probe, the miniaturization of a typical SSTF setup would need to be considered. First, the fluorescence signal would have to be collected by the delivery fiber. With a large mode area fiber or a double-clad photonic crystal fiber [32], these large cores allow for high collection efficiency throughout the depth of focus as the focal plane is scanned. Second, a dichroic mirror placed at an appropriate angle after the grating can redirect the fluorescence signal into the fiber without affecting the excitation beam. The rest of the optical setup (the collimating lens and the objective lens) is the same as that in an endoscope setup and can be similarly miniaturized.

Fast and electronically-controlled GDD scanning is desirable for remote axial scanning. In our preliminary experiments, translating a pair of prisms into the beam 
path adjusts the GDD in the system. Although sufficient for demonstrating the concept of remote axial scanning, it is quite slow because linear translation of a bulky prism is necessary. Faster GDD scanning can be realized based on the same principle except by scanning the optical beam instead of moving the prisms. A regular scan mirror positioned at the focal point of a lens can quickly (up to $\mathrm{kHz}$ ) redirect the excitation beam so that the amount of prism glass in the beam path varies, changing the GDD and the chirp of the pulse. In general, axial scanning is done in discrete steps. Thus, an array of varying length glass rods could replace the prisms in order to scan through a range of fixed GDD values. An alternative method would be to manipulate the phase of the beam after the grating using a spatial light modulator [33, 34], multi-element deformable mirror [35], or acousto-optic modulator [36, 37],

allowing for fast non-mechanical scanning of the focal plane. Chapters 4 and 5 detail two new approaches that are low cost, high speed, broadband, and can tolerate higher intensities: a rotating cylindrical lens [38] and a single-element piezo bimorph mirror [39].

\subsection{Conclusion}

We have shown that SSTF allows for shifting the temporal focal plane along the optical axis by adjusting the dispersion in the system. In the limit that GDD is small, the shift of the temporal focal plane is linearly dependent upon dispersion, and the pulse width is transform-limited. When the temporal focus occurs outside of the geometric overlap of the beams, this relationship breaks down, resulting in a maximum scanning range that is proportional to the axial resolution. We also demonstrated that this method of axial scanning works well for a fiber delivery system, allowing remote axial scanning without any moving parts at the distal end of 
the fiber. As an added advantage, SSTF automatically compensates for dispersion, allowing fiber delivery systems to recover a transform-limited pulse width at the focus without pre-compensation. We believe a highly promising application of this SSTF technique is an axial-scanning TPEF flexible fiber probe with no moving parts at the distal end.

\section{Acknowledgments}

This research was made possible by Grant Number 1R21CA115472-01 from the National Cancer Institute (NCI), National Institutes of Health. 


\section{REFERENCES}

1. W. Denk, J. H. Strickler, and W. W. Webb, "2-Photon Laser Scanning Fluorescence Microscopy," Science 248, 73-76 (1990).

2. J. C. Jung, and M. J. Schnitzer, "Multiphoton endoscopy," Optics Letters 28, 902-904 (2003).

3. C. Liang, M. R. Descour, K. B. Sung, and R. Richards-Kortum, "Fiber confocal reflectance microscope (FCRM) for in-vivo imaging," Optics Express 9, 821$830(2001)$

4. K. B. Sung, C. N. Liang, M. Descour, T. Collier, M. Follen, and R. RichardsKortum, "Fiber-optic confocal reflectance microscope with miniature objective for in vivo imaging of human tissues," IEEE Transactions on Biomedical Engineering 49, 1168-1172 (2002).

5. A. R. Rouse, A. Kano, J. A. Udovich, S. M. Kroto, and A. F. Gmitro, "Design and demonstration of a miniature catheter for a confocal microendoscope," Applied Optics 43, 5763-5771 (2004).

6. W. Gobel, J. N. D. Kerr, A. Nimmerjahn, and F. Helmchen, "Miniaturized two-photon microscope based on a flexible coherent fiber bundle and a gradient-index lens objective," Optics Letters 29, 2521-2523 (2004).

7. D. L. Dickensheets, and G. S. Kino, "Micromachined scanning confocal optical microscope," Optics Letters 21, 764-766 (1996).

8. U. Hofmann, S. Muehlmann, M. Witt, K. Dorschel, R. Schutz, and B. Wagner, "Electrostatically driven micromirrors for a miniaturized confocal laser scanning microscope," Proceedings of SPIE 3878, 29-38 (1999). 
9. D. Bird, and M. Gu, "Two-photon fluorescence endoscopy with a micro-optic scanning head," Optics Letters 28, 1552-1554 (2003).

10. F. Helmchen, M. S. Fee, D. W. Tank, and W. Denk, "A miniature headmounted two-photon microscope: High-resolution brain imaging in freely moving animals," Neuron 31, 903-912 (2001).

11. D. Kim, K. H. Kim, S. Yazdanfar, and P. T. C. So, "Optical biopsy in highspeed handheld miniaturized multifocal multiphoton microscopy," Proceedings of SPIE 5700, 14-22 (2005).

12. D. Bird, and M. Gu, "Fibre-optic two-photon scanning fluorescence microscopy," Journal of Microscopy-Oxford 208, 35-48 (2002).

13. L. Giniunas, R. Juskaitis, and S. V. Shatalin, "Scanning Fiberoptic Microscope," Electronics Letters 27, 724-726 (1991).

14. A. R. Rouse, and A. F. Gmitro, "Multispectral imaging with a confocal microendoscope," Optics Letters 25, 1708-1710 (2000).

15. B. Berge, and J. Peseux, "Variable focal lens controlled by an external voltage: An application of electrowetting," European Physical Journal E 3, 159-163 (2000).

16. S. Kuiper, and B. H. W. Hendriks, "Variable-focus liquid lens for miniature cameras," Applied Physics Letters 85, 1128-1130 (2004).

17. A. J. Wright, B. A. Patterson, S. P. Poland, J. M. Girkin, G. M. Gibson, and M. J. Padgett, "Dynamic closed-loop system for focus tracking using a spatial light modulator and a deformable membrane mirror," Optics Express 14, 222-228 (2006).

18. H. Suchowski, D. Oron, and Y. Silberberg, "Generation of a dark nonlinear focus by spatio-temporal coherent control," Optics Communications 264, 482-487 (2006). 
19. M. J. Levene, D. A. Dombeck, K. A. Kasischke, R. P. Molloy, and W. W. Webb, "In vivo multiphoton microscopy of deep brain tissue," Journal of Neurophysiology 91, 1908-1912 (2004).

20. B. A. Flusberg, J. C. Lung, E. D. Cocker, E. P. Anderson, and M. J. Schnitzer, "In vivo brain imaging using a portable 3.9 gram two-photon fluorescence microendoscope," Optics Letters 30, 2272-2274 (2005).

21. Y. Yasuno, S. Makita, T. Yatagai, T. F. Wiesendanger, A. K. Ruprecht, and H. J. Tiziani, "Non-mechanically-axial-scanning confocal microscope using adaptive mirror switching," Optics Express 11, 54-60 (2003).

22. D. Oron, E. Tal, and Y. Silberberg, "Scanningless depth-resolved microscopy," Optics Express 13, 1468-1476 (2005).

23. G. Zhu, J. van Howe, M. Durst, W. Zipfel, and C. Xu, "Simultaneous spatial and temporal focusing of femtosecond pulses," Optics Express 13, 2153-2159 (2005).

24. O. E. Martinez, "Grating and prism compressors in the case of finite beam size," J. Opt. Soc. Am. B 3, 929-934 (1986).

25. J. Goodman, Introduction to Fourier Optics (Roberts \& Company, Englewood, Colorado, 2005).

26. D. G. Ouzounov, K. D. Moll, M. A. Foster, W. R. Zipfel, W. W. Webb, and A. L. Gaeta, "Delivery of nanojoule femtosecond pulses through large-core microstructured fibers," Optics Letters 27, 1513-1515 (2002).

27. W. Gobel, A. Nimmerjahn, and F. Helmchen, "Distortion-free delivery of nanojoule femtosecond pulses from a Ti : sapphire laser through a hollow-core photonic crystal fiber," Optics Letters 29, 1285-1287 (2004). 
28. E. Tal, D. Oron, and Y. Silberberg, "Improved depth resolution in video-rate line-scanning multiphoton microscopy using temporal focusing," Optics Letters 30 , 1686-1688 (2005).

29. Y. C. Wu, P. Xi, J. N. Y. Qu, T. H. Cheung, and M. Y. Yu, "Depth-resolved fluorescence spectroscopy of normal and dysplastic cervical tissue," Optics Express 13, 382-388 (2005).

30. M. C. Skala, J. M. Squirrell, K. M. Vrotsos, V. C. Eickhoff, A. GendronFitzpatrick, K. W. Eliceiri, and N. Ramanujam, "Multiphoton microscopy of endogenous fluorescence differentiates normal, precancerous, and cancerous squamous epithelial tissues," Cancer Research 65, 1180-1186 (2005).

31. W. R. Zipfel, R. M. Williams, R. Christie, A. Y. Nikitin, B. T. Hyman, and W. W. Webb, "Live tissue intrinsic emission microscopy using multiphoton-excited native fluorescence and second harmonic generation," Proceedings of the National Academy of Sciences of the United States of America 100, 7075-7080 (2003).

32. L. Fu, A. Jain, H. K. Xie, C. Cranfield, and M. Gu, "Nonlinear optical endoscopy based on a double-clad photonic crystal fiber and a MEMS mirror," Optics Express 14, 1027-1032 (2006).

33. A. M. Weiner, D. E. Leaird, J. S. Patel, and J. R. Wullert, "Programmable Femtosecond Pulse Shaping by Use of a Multielement Liquid-Crystal Phase Modulator," Optics Letters 15, 326-328 (1990).

34. M. M. Wefers, and K. A. Nelson, "Programmable Phase and Amplitude Femtosecond Pulse Shaping," Optics Letters 18, 2032-2034 (1993). 
35. E. Zeek, K. Maginnis, S. Backus, U. Russek, M. Murnane, G. Mourou, H. Kapteyn, and G. Vdovin, "Pulse compression by use of deformable mirrors," Optics Letters 24, 493-495 (1999).

36. P. Tournois, "Acousto-optic programmable dispersive filter for adaptive compensation of group delay time dispersion in laser systems," Optics Communications 140, 245-249 (1997).

37. R. Du, K. Bi, S. Zeng, D. Li, S. Xue, and Q. Luo, "Analysis of fast axial scanning scheme using temporal focusing with acousto-optic deflectors," Journal of Modern Optics 56, 102 (2009).

38. M. E. Durst, D. Kobat, and C. Xu, "Tunable dispersion compensation by a rotating cylindrical lens," Opt. Lett. 34, 1195-1197 (2009).

39. M. E. Durst, and C. Xu, "Kilohertz Tunable Dispersion Compensation with a Bimorph Piezo Deformable Mirror," in Conference on Lasers and ElectroOptics/International Quantum Electronics Conference(Optical Society of America, 2009), p. CMLL6. 


\title{
CHAPTER 4
}

\section{TUNABLE DISPERSION COMPENSATION BY A ROTATING CYLINDRICAL $\mathrm{LENS}^{5}$}

\begin{abstract}
We present a new technique for tunable dispersion compensation that is low cost, high speed, and has a large tuning range. By rotating a cylindrical lens at the Fourier plane of a folded 4-f grating pair system, the group velocity dispersion can be tuned over a range greater than $10^{5} \mathrm{fs}^{2}$, sufficient for compensating the dispersion of several meters of optical fiber.
\end{abstract}

\subsection{Introduction}

Various applications in ultrafast and nonlinear optics require a tunable dispersion compensator. For example, because the fluorescence signal in multiphoton microscopy (MPM) depends inversely on the pulse width, one can maximize the signal by compensating the dispersion along the optical path [1]. Dispersion compensation in MPM is particularly important when highly dispersive elements such as acousto-optic or electro-optic modulators are used [2]. Another application is for simultaneous spatial and temporal focusing (SSTF), where a temporal focus can be scanned axially by tuning the dispersion [3]. Typical methods for compensating dispersion include prism pairs [4], grating pairs [5], or a combination of both with grisms [6], but their tuning speed is limited by the mechanical translation of these bulky optical elements. Other techniques have been developed to simplify these setups by using a single diffractive element in a folded system [7], but ultimately the tuning of a 4-f grating setup is still accomplished by translating the components over large distances [8].

\footnotetext{
${ }^{5}$ The contents of this appendix have been reproduced from Optics Letters, 34, No. 8, 1195 (2009).
} 
Several electronically or thermally tuned devices exist. For fiber applications, thermally-tuned fiber-Bragg gratings [9], electronically-driven phase modulators [10], and waveguide grating routers combined with deformable mirrors or phase modulators [11] can all tune the dispersion at high speed, but these devices cannot support ultrashort pulse propagation. Free-space electronically-addressed devices, such as acousto-optic modulators (AOMs) [12], spatial light modulators (SLMs) [13], and deformable mirrors [14], can achieve programmable and highspeed dispersion tuning. In these devices, a pixelated array can impose a spectral phase with arbitrary shape but at a high cost and with limited tuning range. The advantage of these devices is the capability to compensate for higher-order dispersion, but this is unnecessary for many practical applications. For example, when $50 \mathrm{fs}$ or longer duration pulses are used in MPM [15] and SSTF for remote axial scanning [3], compensation of the second-order group delay dispersion (GDD) is sufficient.

We describe here a new technique for tunable dispersion compensation by using a rotating cylindrical lens. We use a grating, a spherical collimating lens, and a mirror in a folded 4-f system. Our setup is similar to those used with deformable mirrors and reflective SLMs except we place a plano-convex cylindrical lens at the Fourier plane (Figure 4.1). The optical path length variation across the spectral components due to the curvature of the lens imposes a quadratic phase across the spectrum. Rotating the cylindrical lens changes the effective radius of curvature, thus changing the amount of GDD in the system. By using a cylindrical lens of a sufficiently long focal length, a large dispersion tuning range can be achieved while introducing negligible spatial distortion due to the focusing effect of the cylindrical lens. Because the cylindrical lens is rotated rather than linearly translated, the output beam exhibits directional stability, and the motion can be made high speed with a 


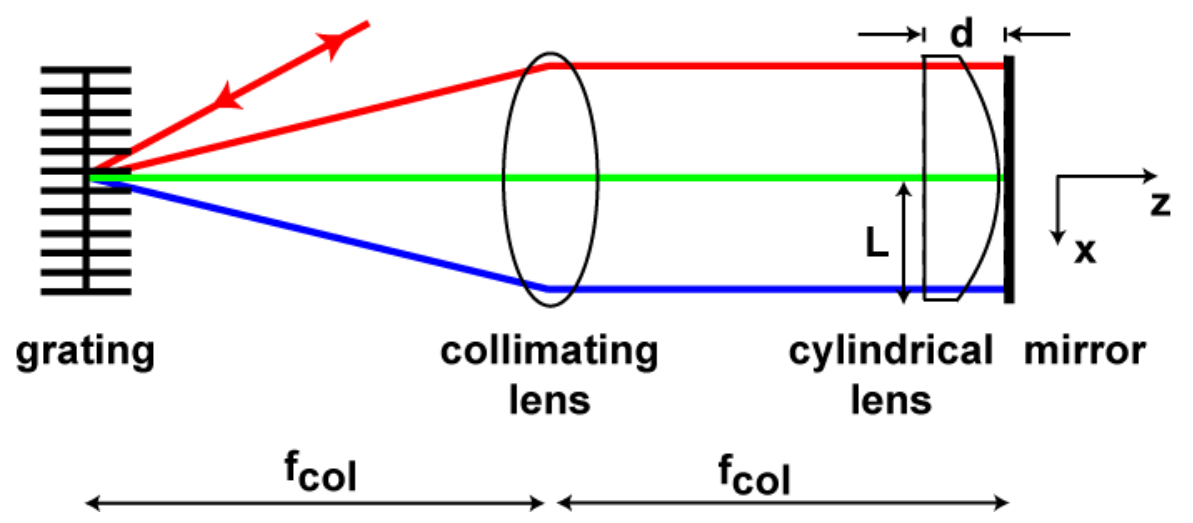

Figure 4.1. GDD compensation device: folded 4-f grating pair setup with a cylindrical lens at the Fourier plane. 
simple motor. Thus, high-speed tunable dispersion compensation is realized by rotating a cylindrical lens in a 4-f grating-pair setup. Our technique is low cost, high speed, and has a large range that is sufficient for compensating the dispersion of several meters of optical fiber.

\subsection{Theory}

A glass plano-convex lens is shown at the Fourier plane of a folded 4-f grating pair setup in Figure 4.1. We derive an expression for the optical path length due to the curvature of the lens as a function of the transverse position $x$ :

$$
z=n \cdot d+(n-1)\left[\sqrt{R^{2}-x^{2}}-R\right]
$$

where $z$ is the path length, $n$ is the refractive index of the glass, $d$ is the thickness of the lens at its center, $R$ is the radius of curvature of the lens, and $x$ is the distance from the center of the lens. Dropping the constant phase term and assuming that the focal length of the lens is large ( $R \gg x)$, Eq. (4.1) can be written as:

$$
z \approx-(n-1) \frac{x^{2}}{2 R}=-\frac{x^{2}}{2 f_{c y l}}
$$

where $f_{c y l}$ is the focal length of the cylindrical lens. If the cylindrical lens is rotated about the optical axis, a simple coordinate rotation, taking into account the quadratic spatial profile of the lens, allows the effective focal length to be written

as $f_{\text {eff }}=f_{c y l} / \cos ^{2} \theta$, where $\theta$ is the rotation angle of the lens about the optical axis.

Taking into account the double-pass configuration, the phase delay due to the thickness of the lens can be related to the GDD by:

$$
-\frac{k \cdot x^{2}}{f_{\text {eff }}}=\frac{1}{2} G D D \cdot\left(x \frac{\Omega}{2 L}\right)^{2}
$$

where $k$ is the wavevector corresponding to the center wavelength $(\lambda)$ and $L$ is the half width of the lens. $\Omega$ is the total optical bandwidth across the cylindrical lens, which is determined by the incident angle, the groove density of the grating, and the focal 
length of the collimating lens $\left(f_{c o l}\right)$. We note that $\Omega$ is independent of the pulse bandwidth. Solving for the GDD:

$$
G D D=-\frac{2 k}{f_{\text {eff }}}\left(\frac{2 L}{\Omega}\right)^{2}=-\frac{4 \pi}{\lambda f_{c y l}}\left(\frac{2 L}{\Omega} \cos \theta\right)^{2}
$$

As shown in Eq. (4.4), the amount of GDD imparted on the beam can be tuned by rotating the cylindrical lens. For $f_{c y l}=10 \mathrm{~m}, L=2.54 \mathrm{~cm}, \lambda=775 \mathrm{~nm}$, and $\Omega=1.79 \mathrm{x}$ $10^{14} \mathrm{~Hz}\left(f_{c o l}=30 \mathrm{~cm}\right.$, a grating with a groove density of 1800 lines per mm, and a $39^{\circ}$ incident angle on the grating), the maximum GDD will be $-1.3 \times 10^{5} \mathrm{fs}^{2}$.

\subsection{Experiment}

The experimental setup to characterize the GDD compensation device is shown in Figure 4.1. We use a mode-locked Ti:Sapphire laser (Spectra-Physics) centered at $775 \mathrm{~nm}$ with approximately $10 \mathrm{~nm}$ (FWHM) of bandwidth. A ruled diffraction grating with a groove density of 1800 lines per mm separates the beam into its monochromatic components, which are then collimated by a spherical lens $\left(f_{\text {col }}=\right.$ $30 \mathrm{~cm})$. At the Fourier plane, we place a folding mirror and a plano-convex cylindrical lens $\left(f_{c y l}=10 \mathrm{~m}, \mathrm{CVI}\right.$ Melles Griot SCX-50.8-5000.0-C) on a rotation stage. We measure the pulse width of the beam exiting the GDD compensation device using an interferometric second-order autocorrelator, which has a GaAsP diode as the nonlinear element. The pulse width for different rotation angles of the cylindrical lens are shown in Figure 4.2. We were able to broaden the pulse from $80 \mathrm{fs}$ with no curvature $\left(\theta=90^{\circ}\right)$ to $3.3 \mathrm{ps}$ with full curvature $\left(\theta=0^{\circ}\right.$, see autocorrelation trace in Figure 4.3(a)), corresponding to an induced GDD of $-1.26 \times 10^{5} \mathrm{fs}^{2}$ (sech shape). Note that the pulse width follows a cosine-squared dependence on rotation angle (solid curve in Figure 4.2). The experimentally-obtained GDD tuning range and the dependence on the rotation angle $\theta$ agree well with the theoretical predictions of Eq. (4.4). To 
demonstrate the utility of the device for fiber delivery, we couple the beam into 2 meters of standard single-mode fiber (SSMF), which has an estimated dispersion value of $7.6 \times 10^{4} \mathrm{fs}^{2}$ at this wavelength. The coupled power into the SSMF was kept sufficiently low to avoid any fiber nonlinearity. By rotating the cylindrical lens, we are able to recover an 80 fs pulse out of the fiber (see autocorrelation trace in Figure $4.3(\mathrm{~b}))$.

To determine the spatial and temporal quality of the pulses, we measure the dependence of the two-photon induced photocurrent by focusing the beam onto a GaAsP photodiode through $90^{\circ}$ rotation of the cylindrical lens (Figure 4.4(a)). The two-photon induced current closely follows the expected inverse pulse-width dependence, as shown by the solid curve in Figure 4.4(a). It is well known that the amount of two-photon excitation depends sensitively on the spatial and temporal quality of the excitation beam. Results in Figure 4.4(a) indicate that the spatial and temporal quality of the pulse is not degraded after the dispersion compensation device. To demonstrate the superb beam pointing stability of this setup while tuning the GDD, we couple the output beam into a SSMF and measure the output power as a function of rotation angle $\theta$ without changing the fiber coupling setup (Figure 4.4(b)). The output power varies by approximately $2.5 \%$ throughout the GDD tuning range. We also measure the point spread function (PSF) of the output beam by imaging $200 \mathrm{~nm}$ fluorescent beads in a two-photon laser scanning microscope. Using a $0.57 \mathrm{NA}$ objective lens, the lateral FWHM of the bead image is 0.5 microns, and the axial FWHM is 3.6 microns, which is in agreement with theory [1]. Because a rotation angle of $0^{\circ}$ dramatically broadens the pulse, a $40 \mathrm{~cm}$ SF11 glass rod is added to the beam path to partially negate the pulse broadening and increase the signal at $\theta=0^{\circ}$. The PSF does not change size or shape as we rotate the cylindrical lens from $0^{\circ}$ to $90^{\circ}$ (Figure 4.4(a) insets), thus demonstrating its suitability for imaging applications. 


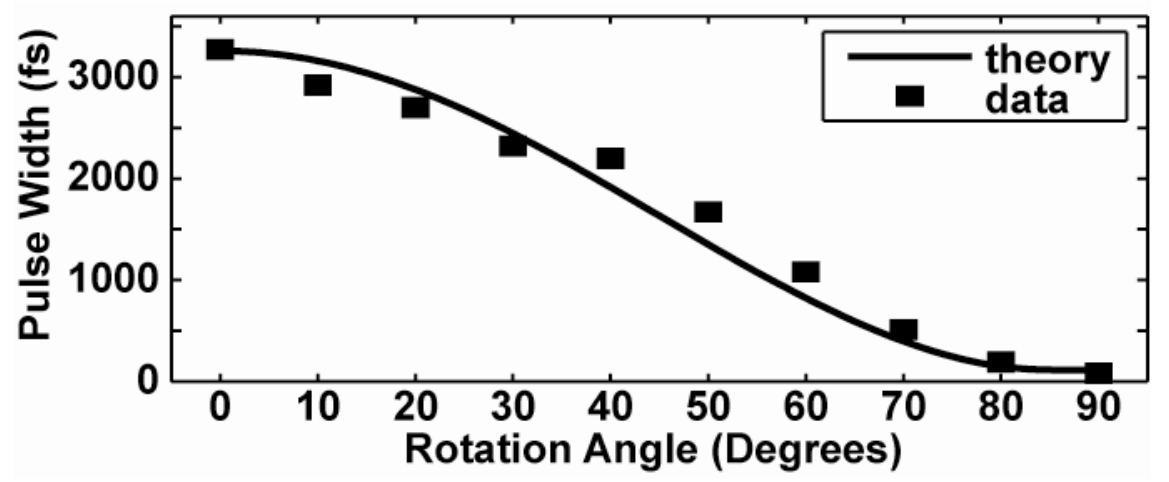

Figure 4.2. Pulse width versus rotation angle of the cylindrical lens. Squares: autocorrelation data. Solid line: theoretical curve based on Eq. (4.4). 


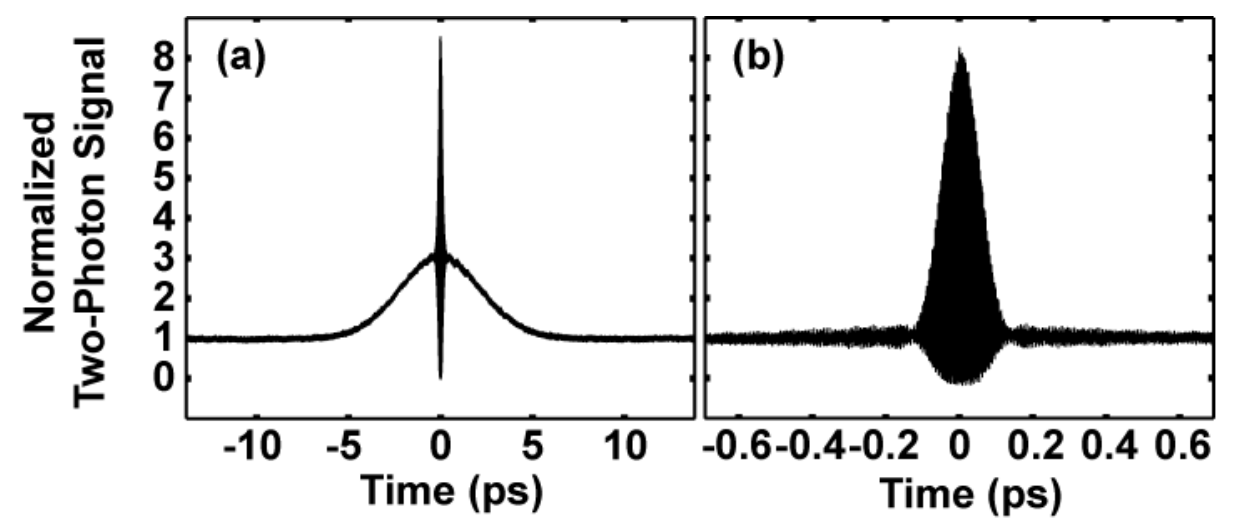

Figure 4.3. Interferometric second-order autocorrelations of the pulse exiting the dispersion compensation device (a) with maximum curvature $\left(\theta=0^{\circ}\right)$ and (b) with 2 meters of SSMF at $\theta=30^{\circ}$. 


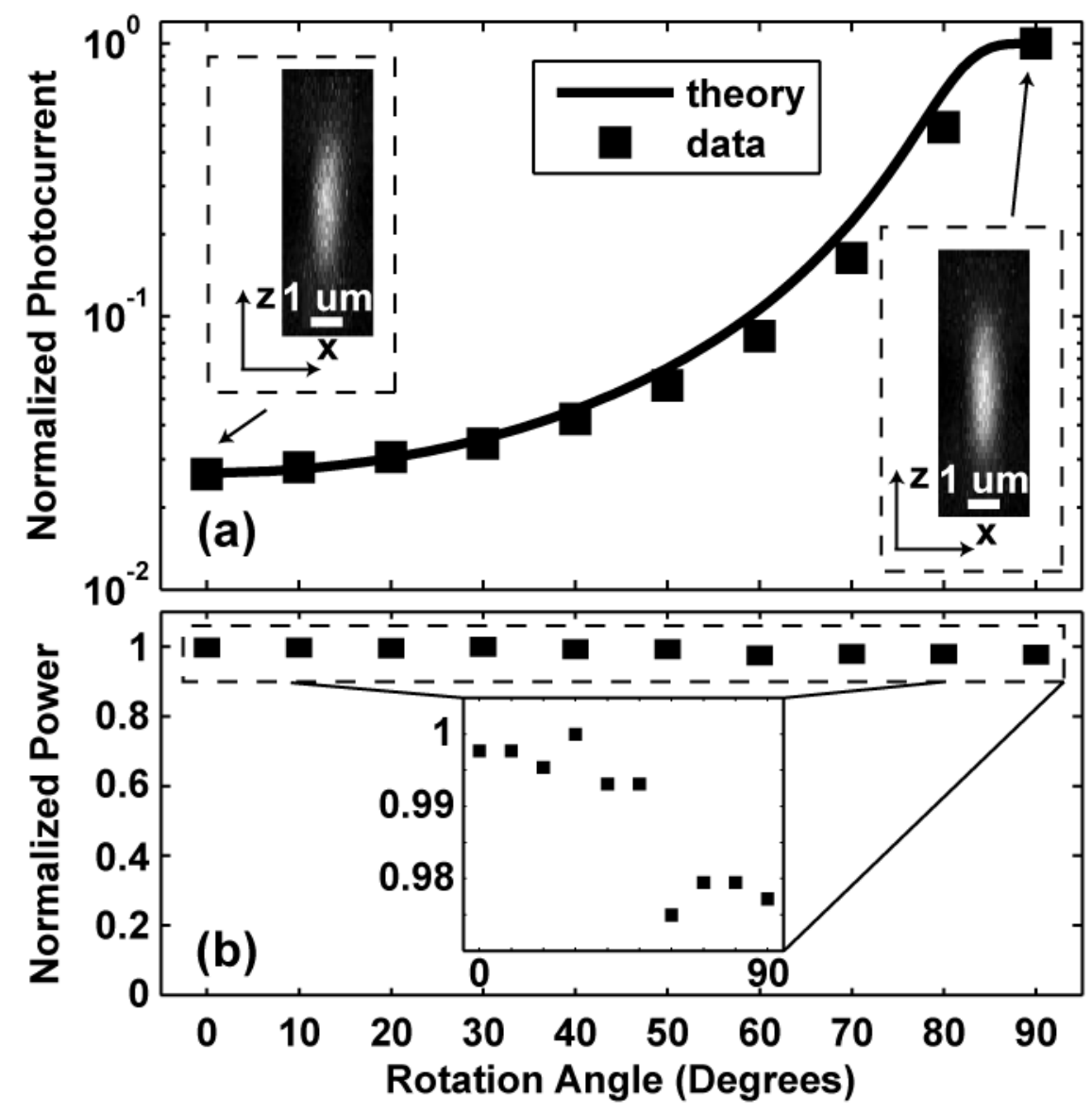

Figure 4.4. (a) Normalized two-photon induced current (log scale) as a function of the rotation angle of the cylindrical lens. Insets: Normalized PSF of output beam at $\theta=0^{\circ}$ and $\theta=90^{\circ}$. (b) Normalized power coupled into a SSMF as a function of cylindrical lens angle. Inset: enlarged view of the power variation. 
Spatial-temporal coupling is a concern in such a 4-f setup, similar to dispersion tuning devices using deformable mirrors or SLMs $[13,14]$. Our measurements show that the bandwidth coupled into a SSMF varied by approximately $20 \%$ over the entire GDD tuning range when the input pulse bandwidth is increased to $17 \mathrm{~nm}$ (FWHM), indicating non-negligible spatial chirp due to the curvature of the cylindrical lens. For our experimental bandwidth of $10 \mathrm{~nm}$, the bandwidth coupled into SSMF varies within $1 \mathrm{~nm}$ throughout the tuning range. While such spatial-temporal coupling can be reduced by using a longer focal length cylindrical lens, the GDD tuning range will be proportionally reduced (Eq. (4.3)). Thus, there is a compromise between the maximum GDD tuning range and the shortest pulse duration. As shown by the experimental results, we were able to obtain $1.26 \times 10^{5} \mathrm{fs}^{2}$ GDD tuning with an $80 \mathrm{fs}$ pulse in our particular setup.

It is informative to compare the performance of our GDD compensator with existing tunable-GDD products that employ AOMs and prism pair configurations. Folded prism-pair designs are low cost but are limited in range by the size of the prism and in speed by the mass of the folding mirror. With AOMs as the dispersive elements, the GDD can be tuned at high-speed, but the available tuning range is similar to the prism-pair design with an order of magnitude greater cost. Our components are low cost, available off the shelf, and yield a broad tuning range of greater than $10^{5} \mathrm{fs}^{2}$. We further note that the sign of the dispersion in our device can be changed by switching from a convex to a concave lens. A fixed offset of the tuning range can also be obtained by adding an extra fixed lens at the Fourier plane. Furthermore, an all-reflecting geometry can be accomplished with a spherical collimating mirror and a rotating cylindrical mirror. 


\subsection{Conclusion}

In this chapter, we have demonstrated a new technique for tunable dispersion compensation by rotating a cylindrical lens at the Fourier plane of a folded 4-f grating

pair system. The GDD can be tuned over a range greater than $10^{5} \mathrm{fs}^{2}$, sufficient for compensating the dispersion of several meters of SSMF. This tunable GDD compensator is low cost, capable of high speeds, and has a large tuning range.

\section{Acknowledgments}

This research was made possible by Grant Number R21CA129648 from the National Institutes of Health. 


\section{REFERENCES}

1. W. R. Zipfel, R. M. Williams, and W. W. Webb, "Nonlinear magic: multiphoton microscopy in the biosciences," Nature Biotechnology 21, 1368-1376 (2003).

2. V. Iyer, B. E. Losavio, and P. Saggau, "Compensation of spatial and temporal dispersion for acousto-optic multiphoton laser-scanning microscopy," Journal of Biomedical Optics 8, 471 (2003).

3. M. E. Durst, G. Zhu, and C. Xu, "Simultaneous spatial and temporal focusing in nonlinear microscopy," Optics Communications 281, 1805 (2008).

4. R. L. Fork, O. E. Martinez, and J. P. Gordon, "Negative Dispersion Using Pairs of Prisms," Optics Letters 9, 150-152 (1984).

5. O. E. Martinez, "3000 Times Grating Compressor with Positive GroupVelocity Dispersion - Application to Fiber Compensation in 1.3-1.6 Mu-M Region," IEEE Journal of Quantum Electronics 23, 59-64 (1987).

6. E. A. Gibson, D. M. Gaudiosi, H. C. Kapteyn, R. Jimenez, S. Kane, R. Huff, C. Durfee, and J. Squier, "Efficient reflection grisms for pulse compression and dispersion compensation of femtosecond pulses," Opt. Lett. 31, 3365 (2006).

7. S. Akturk, X. Gu, M. Kimmel, and R. Trebino, "Extremely simple single-prism ultrashort- pulse compressor," Opt. Express 14, 10108 (2006).

8. Y. Chen, and X. Li, "Dispersion management up to the third order for real-time optical coherence tomography involving a phase or frequency modulator," Opt. Express 12, 5978 (2004).

9. N. Q. Ngo, S. Y. Li, R. T. Zheng, S. C. Tjin, and P. Shum, "Electrically tunable dispersion compensator with fixed center wavelength using fiber Bragg grating," Lightwave Technology, Journal of 21, 1575 (2003). 
10. J. van Howe, and C. Xu, "Ultrafast optical signal processing based upon spacetime dualities," Lightwave Technology, Journal of 24, 2662 (2006).

11. D. M. Marom, C. R. Doerr, M. A. Cappuzzo, C. Evans Yifan, A. Wong-Foy, L. T. Gomez, and S. Chandrasekhar, "Compact colorless tunable dispersion compensator with $1000-\mathrm{ps} / \mathrm{nm}$ tuning range for $40-\mathrm{gb} / \mathrm{s}$ data rates," Lightwave Technology, Journal of 24, 241 (2006).

12. P. Tournois, "Acousto-optic programmable dispersive filter for adaptive compensation of group delay time dispersion in laser systems," Optics Communications 140, 245-249 (1997).

13. A. M. Weiner, D. E. Leaird, J. S. Patel, and J. R. Wullert, "Programmable Femtosecond Pulse Shaping by Use of a Multielement Liquid-Crystal Phase Modulator," Optics Letters 15, 326-328 (1990).

14. E. Zeek, K. Maginnis, S. Backus, U. Russek, M. Murnane, G. Mourou, H. Kapteyn, and G. Vdovin, "Pulse compression by use of deformable mirrors," Optics Letters 24, 493-495 (1999).

15. J. B. Guild, C. Xu, and W. W. Webb, "Measurement of group delay dispersion of high numerical aperture objective lenses using two-photon excited fluorescence," Appl. Opt. 36, 401 (1997). 


\title{
CHAPTER 5
}

\section{KILOHERTZ TUNABLE DISPERSION COMPENSATION IN A TEMPORAL}

\section{FOCUSING SETUP}

\begin{abstract}
We present a technique for tunable group-delay dispersion (GDD) compensation with $>10^{5} \mathrm{fs}^{2}$ range and $2 \mathrm{kHz}$ tuning speed. By using a single-element piezo bimorph mirror, an applied voltage makes the piezo bimorph bend into a parabola. When placed at the Fourier plane in a folded 4-f grating pair setup, a piezo bimorph mirror imposes tunable GDD onto the pulse. In a simultaneous spatial and temporal focusing setup, GDD tuning scans the temporal focal plane axially. By rapidly varying the GDD, we demonstrate high-speed axial scanning at a rate of $2 \mathrm{kHz}$ over a range of 100 microns.
\end{abstract}

\subsection{Introduction}

A dispersion compensator with a large tuning range has applications in ultrafast and nonlinear phenomena. In multiphoton microscopy (MPM), the fluorescence signal depends inversely on the pulse width. By compensating the dispersion, one can maximize the signal [1]. Dispersion compensation in MPM is particularly important when highly dispersive acousto-optic scanners are used [2]. In simultaneous spatial and temporal focusing (SSTF), axial confinement is generated by spatially separating the frequency components of a pulse with a grating and recombining them with an objective lens, creating a temporal focus at the geometric focal plane $[3,4]$. When there is residual group-delay dispersion (GDD) in the system, the temporal focus, i.e. the axial position where the pulse width is shortest, becomes offset from the geometric focus $[5,6]$. By rapidly varying the GDD, the temporal focal plane can be scanned axially at high speeds $[3,4,7]$. 
Typical methods for dispersion tuning include prism pairs [8], grating pairs [9], or a combination of both with grisms [10], but their tuning speed is limited by the mechanical translation of these bulky optical elements. Electronically addressed devices, such as acousto-optic modulators (AOMs) [7, 11], spatial light modulators (SLMs) [12], and multi-element deformable membrane mirrors [13, 14], can achieve programmable and high-speed dispersion tuning. In these devices, a pixilated array is placed at the Fourier plane of a folded 4-f grating pair setup, where the spatial path length difference provides the desired variation in spectral phase. These programmable devices can generate spectral phase with arbitrary shape but at a high cost and with limited tuning range. For SSTF, however, their ability to generate higher-order dispersion is unnecessary, because tuning of the second-order groupdelay dispersion is sufficient.

We seek a simple GDD tuning device which provides a quadratic shape at the Fourier plane of a 4-f grating pair setup. This has been demonstrated with a rotating cylindrical lens placed at the Fourier plane [15], but stable, high-speed rotation of such a large optical element has not been demonstrated. Tunable GDD has been demonstrated using a single-element deformable membrane mirror but with limited range (on the order of $10^{2} \mathrm{fs}^{2} \mathrm{GDD}$ ) [16]. Piezoelectric bimorphs assume a quadratic shape with an applied voltage, allowing for large curvatures, high-speed tuning, and electronic control $[17,18]$. The first demonstrations of pulse shaping with a piezoelectric bimorph utilized multiple electrodes to independently address multiple sectors of the actuator [19]. In this chapter, we describe a technique for generating high-speed tunable GDD by using a single-element piezo bimorph mirror. The use of a single piezo bimorph not only makes the device cost-effective but also provides large quadratic curvatures (i.e., GDD tuning range), circumventing the stroke limitations of multi-element deformable mirrors. Our technique is low cost, high 
speed, broadband, can tolerate high intensities, and has a wide GDD-tuning range of more than $10^{5} \mathrm{fs}^{2}$. When applied to an SSTF setup, this technique will allow for high speed axial scanning. SSTF axial scanning has been performed with prisms [5], AODs [7], and SLMs [6], but the reported axial scanning speed is low. In this chapter, we demonstrate high-speed axial scanning over 100 microns at a rate of $2 \mathrm{kHz}$ in an SSTF setup.

\subsection{Theory}

We analyze theoretically a GDD tuning technique that uses a grating, a spherical collimating lens, and a deformable piezo bimorph mirror in a folded 4-f system (Figure 5.1). The optical path length variation due to the parabolic curvature of the mirror imposes a quadratic phase across the spectrum. When the piezo bimorph is connected in series and fixed at its center, the bending of the piezo bimorph can be characterized by $[17,18,20]$ :

$$
y=-\frac{3 \cdot d_{31}}{2 \cdot T^{2}} V \cdot x^{2}
$$

where $y$ is the axial displacement of the piezo, $x$ is the lateral position of the piezo with respect to the optical axis, $V$ is the applied voltage, $d_{31}$ is the piezoelectric coefficient, and $T$ is the total thickness of the piezo. From Eq. (5.1), the path length traveled by an individual monochromatic beam is $2 y$ due to the double-pass configuration. Because the grating maps the individual frequency components to distinct transverse positions, the quadratic path length difference can be converted to quadratic spectral phase, or GDD:

$$
\begin{aligned}
2 k \cdot y & =\frac{1}{2} G D D \cdot \omega^{2} \\
-2\left(\frac{2 \pi}{\lambda}\right) \frac{3 \cdot V \cdot d_{31}}{2 \cdot T^{2}} x^{2} & =\frac{1}{2} G D D \cdot\left(x \frac{\omega_{T O T}}{L}\right)^{2}
\end{aligned}
$$

where $k$ is the wavevector corresponding to the center wavelength $(\lambda)$. $\omega_{\text {TOT }}$ is the total 


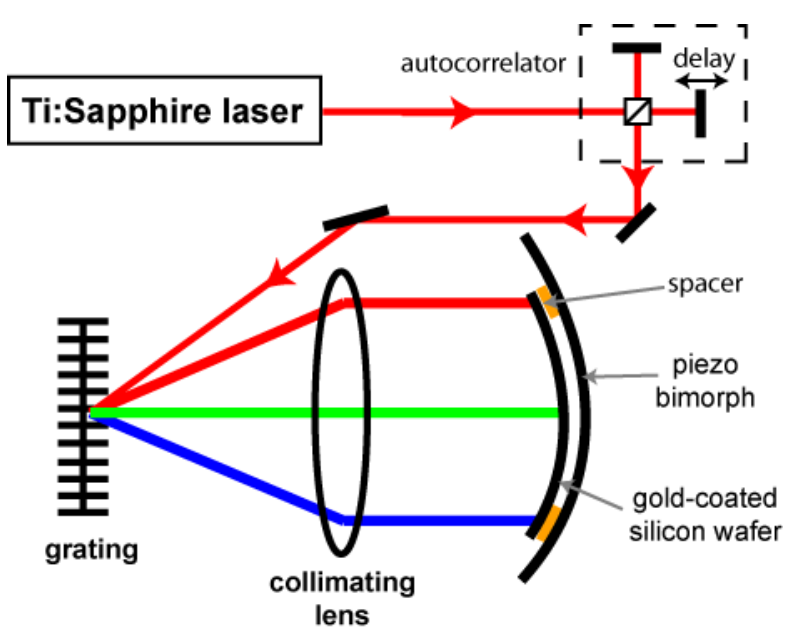

Fig 5.1: GDD compensation setup consisting of a folded 4-f grating pair setup with a single piezo bimorph mirror at the Fourier plane. 
optical bandwidth across the piezo bimorph mirror (of total width $L$ ), which is determined by the incident angle, the groove density of the grating, and the focal length of the collimating lens $\left(f_{\mathrm{col}}\right)$. We note that $\omega_{\text {TOT }}$ is independent of the pulse bandwidth. Solving for the GDD:

$$
G D D=-\frac{12 \pi}{\lambda} \frac{V \cdot d_{31}}{T^{2}}\left(\frac{L}{\omega_{\text {TOT }}}\right)^{2}
$$

As shown in Eq. (5.3), the amount of GDD imparted on the beam can be tuned by changing the piezo bimorph drive voltage. For $V=100 \mathrm{~V}, d_{31}=-1.9 \times 10^{-10} \mathrm{~m} / \mathrm{V}, T=0.51$ $\mathrm{mm}, L=5.08 \mathrm{~cm}, \lambda=775 \mathrm{~nm}$, and $\omega_{\mathrm{TOT}}=1.79 \times 10^{14} \mathrm{~Hz}\left(f_{\text {col }}=30 \mathrm{~cm}\right.$, a grating with a groove density of 1800 lines per $\mathrm{mm}$, and a $39^{\circ}$ incident angle on the grating), the imposed GDD will be $2.8 \times 10^{5} \mathrm{fs}^{2}$.

\subsection{Experiment}

The experimental setup for the GDD tuning device is shown in Figure 5.1. A ruled diffraction grating (1800 lines $/ \mathrm{mm})$ spectrally separates the beam from a modelocked Ti:Sapphire laser $\left(\lambda_{0}=775 \mathrm{~nm}, \Delta \lambda=8 \mathrm{~nm}\right)$, which is then collimated by a spherical lens $\left(\mathrm{f}_{\mathrm{col}}=30 \mathrm{~cm}\right)$. At the focal plane of the collimating lens, we place a piezo bimorph (T220-A4-503X, Piezo Systems, Inc.) which is fixed at its center, allowing it to bend both forwards and backwards. A 380-micron thick pre-polished silicon wafer (University Wafer) is coated with gold to achieve high reflectivity. The silicon mirror is then mounted onto the piezo bimorph with double-sided Scotch tape as spacers. The piezo bimorph is driven by a voltage source combined with a linear amplifier (Piezo Systems, Inc.). Changing the drive voltage of the piezo bimorph changes the radius of curvature, thus effectively changing the amount of GDD. Normal and anomalous dispersions can be obtained in the same setup by changing the sign of the drive voltage. 
To determine the amount of GDD imposed by the piezo bimorph mirror, we measure the pulse width of the beam exiting the grating pair using an interferometric second-order autocorrelator. The nonlinear element is a GaAsP photodiode. We couple the beam out of the GDD device using a 50/50 beamsplitter. We are able to broaden a 100 fs pulse to 4.2 ps by varying the drive voltage between $+/-100 \mathrm{~V}$ (Figure 5.2(b)-(d)). Assuming a sech ${ }^{2}$ shape, the piezo mirror produces a maximum GDD of $+/-2.5 \times 10^{5} \mathrm{fs}^{2}$ (Figure 5.2(a)), which agrees well with theory (Eq. (5.3)). The GDD data is not centered about zero applied voltage because the silicon wafer is not mounted perfectly flat with respect to the piezo bimorph.

To demonstrate its high-speed GDD tuning capability, the piezo bimorph is driven with a $1 \mathrm{kHz}$ sine wave. The output beam is then focused onto a GaAsP photodiode, and the resulting two-photon induced photocurrent is measured. The twophoton signal is inversely proportional to the pulse width, allowing real time monitoring of the rapidly varying GDD. Because the sinusoid covers the full range of GDD values twice per period (green curve in Figure 5.3), the nonlinearly excited photocurrent is modulated at a $2 \mathrm{kHz}$ rate due to the time-varying broadening of the pulse width (blue curve in Figure 5.3). We note that we are operating the piezo bimorph above its measured resonance of $160 \mathrm{~Hz}$, thus limiting the maximum GDD value attainable at high speeds (in this chapter, $8.4 \times 10^{4} \mathrm{fs}^{2}$ with $1 \mathrm{kHz}$ applied voltage). At modulation rates lower than $100 \mathrm{~Hz}$, the maximum GDD value is comparable to a DC applied voltage (Figure 5.2(a)).

We incorporate this high-speed GDD tuning device into a typical SSTF setup [3]. The beam out of the GDD device impinges upon a ruled diffraction grating (1200 lines $/ \mathrm{mm})$. The $0^{\text {th }}$ order reflection is focused onto a GaAsP diode to monitor the GDD imparted by the tuning device. The $1^{\text {st }}$ order diffraction is used to perform SSTF, in which it is collimated by a $40 \mathrm{~cm}$ focal length lens and focused by an 

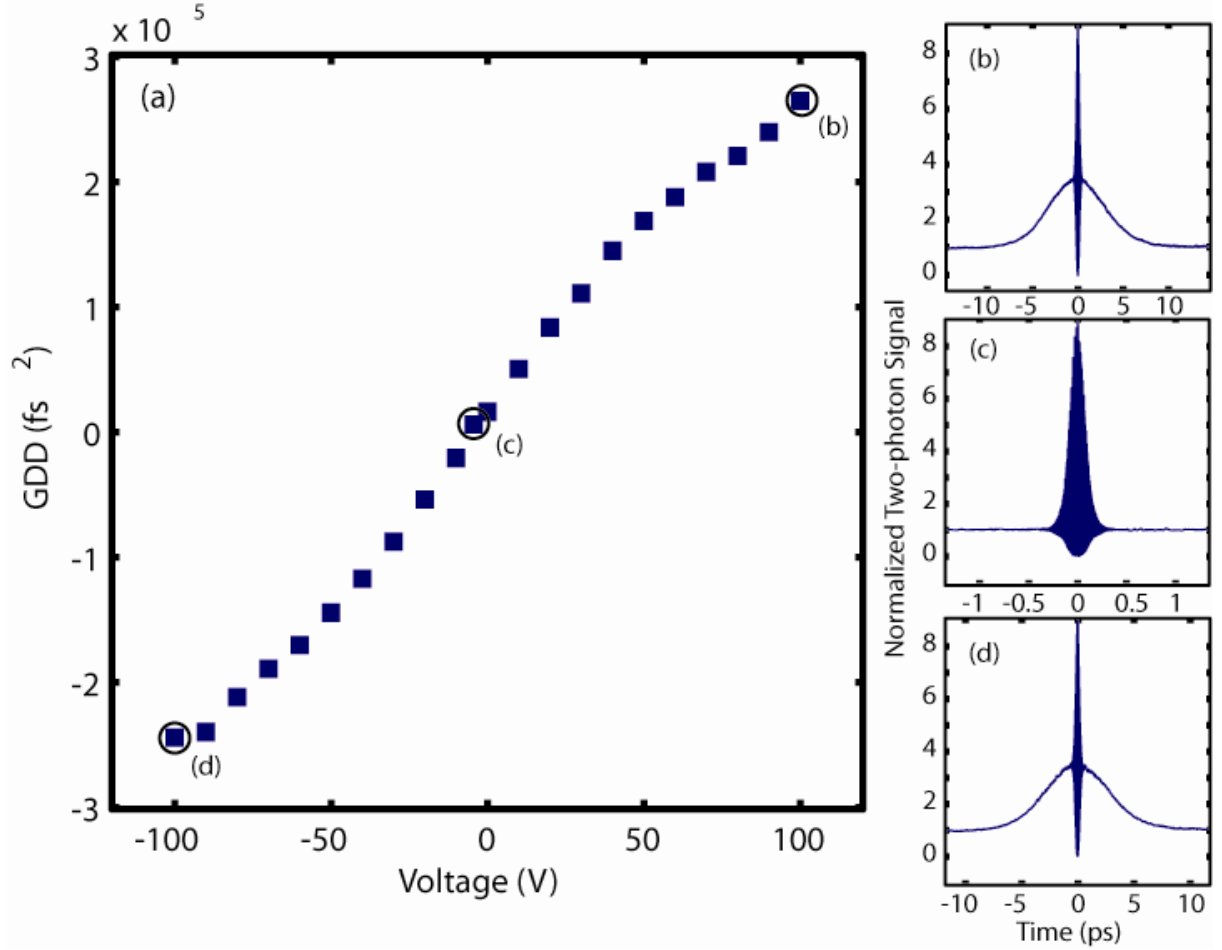

Figure 5.2: (a) Plot of measured GDD versus drive voltage. (b)-(d) Second-order interferometric autocorrelation traces for drive voltages of (b) $100 \mathrm{~V}$, (c) -10 V, (d) $100 \mathrm{~V}$. 


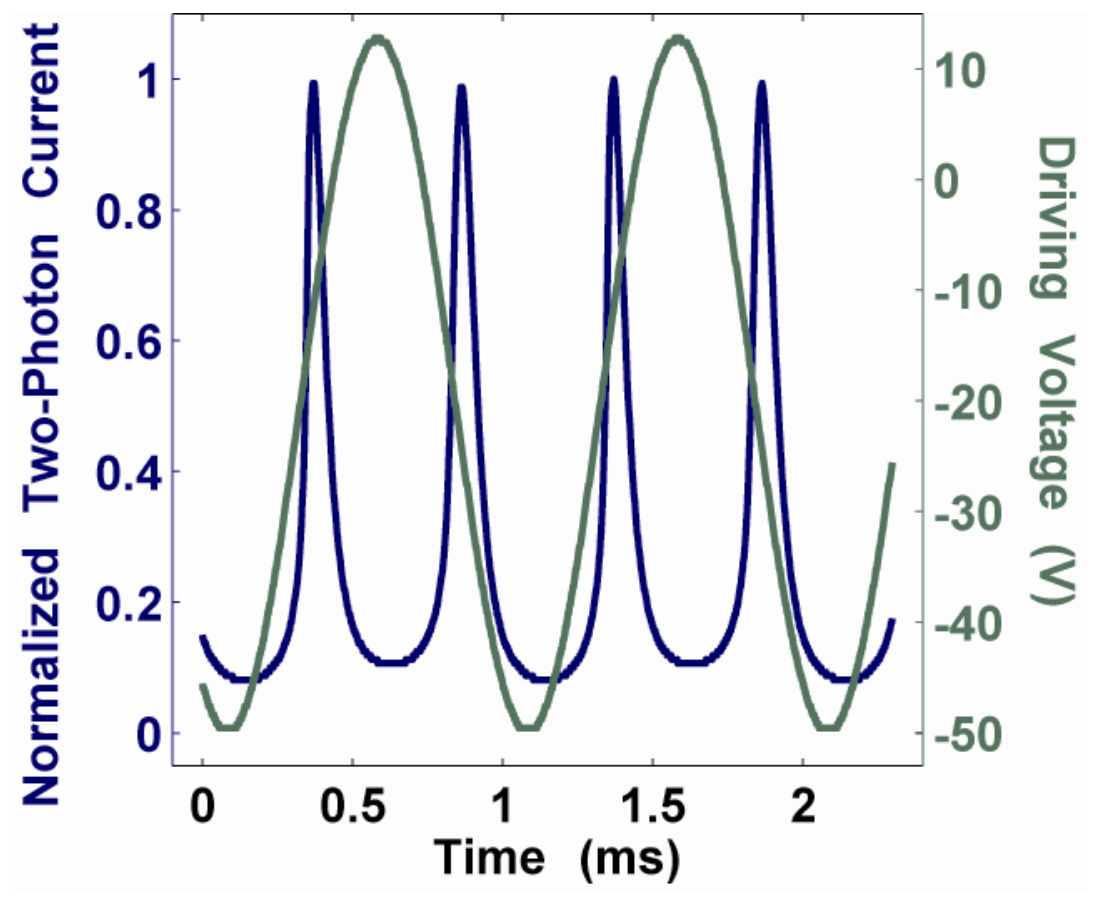

Figure 5.3: Two-photon induced photocurrent (blue curve) and drive voltage (green curve) as a function of time. 
objective (Zeiss Fluar 20x/0.75NA). We place at the geometric focus a thin film of Rhodamine B (1 $\mu \mathrm{m}$ thick) on a cover slip. The two-photon excited fluorescence (TPEF) is directed onto a bialkali photomultiplier tube (PMT, Hamamatsu HC125-02) after passing through a dichroic mirror and emission filters to further remove the excitation light. The electrical pulses from the PMT are integrated using a $10 \mathrm{kHz}$ low pass filter (Stanford Research Systems SR560). When the GDD is zero, the temporal focus is located on top of the slide, yielding a maximum SSTF signal. We then vary the GDD with a $1 \mathrm{kHz}$ sinusoidal drive voltage (green curve in Figure 5.4), generating high-speed axial scanning of the temporal focal plane at a rate of $2 \mathrm{kHz}$ (blue curve in Figure 5.4).

It has been demonstrated that mechanically sweeping a sample through a temporal focus is equivalent to scanning the temporal focus by varying the GDD [5]. Figure 5.5 shows such an axial scan by mechanically translating the sample at a fixed GDD value (black curve in Figure 5.5). We then tune the GDD using our device while fixing the sample at the geometric focal plane (red curve in Figure 5). Because the relationship between the GDD and the shift of the temporal focus is known [5], a lookup table is used to convert the time axis for the data in Figure 5.4 to position in Figure 5.5. Axial scanning by both mechanical translation and GDD tuning achieve nearly identical axial profiles (Figure 5.5). The large dispersion tuning range achieved by our device enabled $100 \mu \mathrm{m}$ of scanning range at a $2 \mathrm{kHz}$ rate with an axial resolution of $20 \mu \mathrm{m}(\mathrm{FWHM})$. This scanning range can be readily increased by decreasing the modulation rate of the applied voltage.

\subsection{Discussion and Conclusion}

We have shown that we can rapidly shift the temporal focal plane axially by varying the GDD at high speeds. This technique can be applied to a fiber probe, 


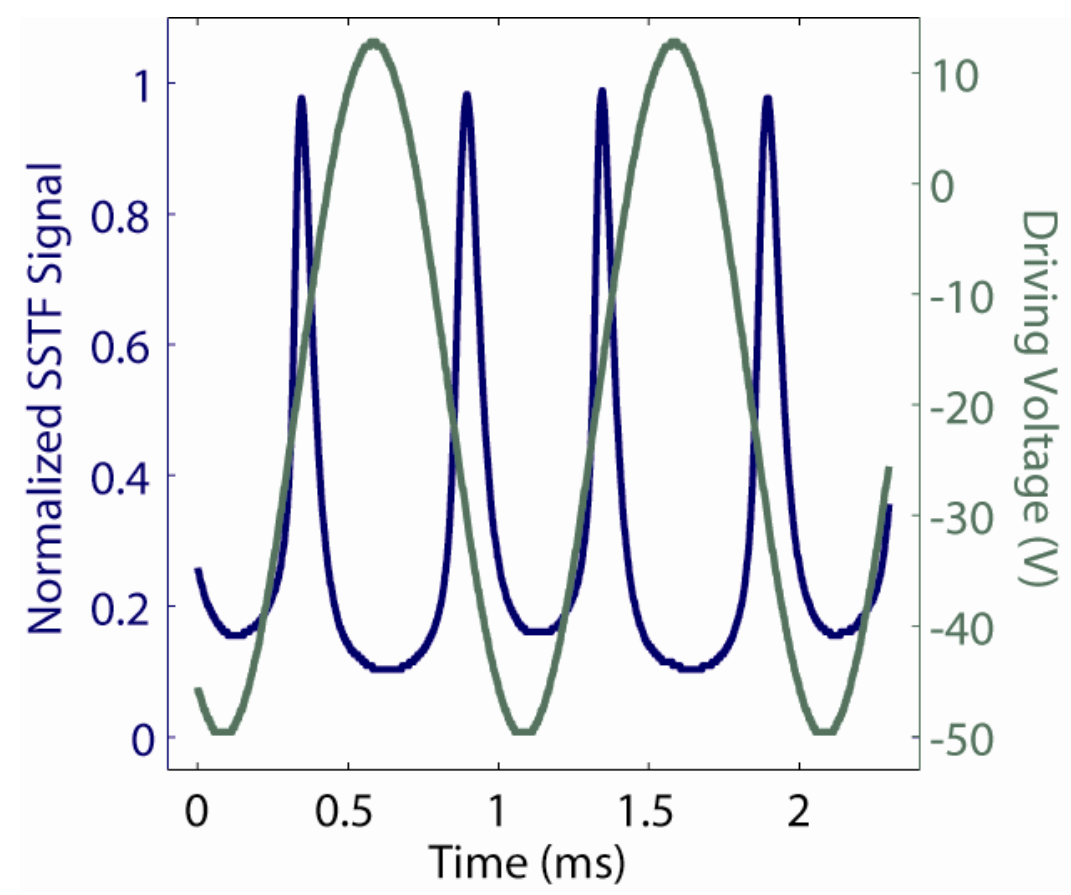

Figure 5.4: High-speed SSTF signal (blue curve) and drive voltage (green curve) as a function of time. 


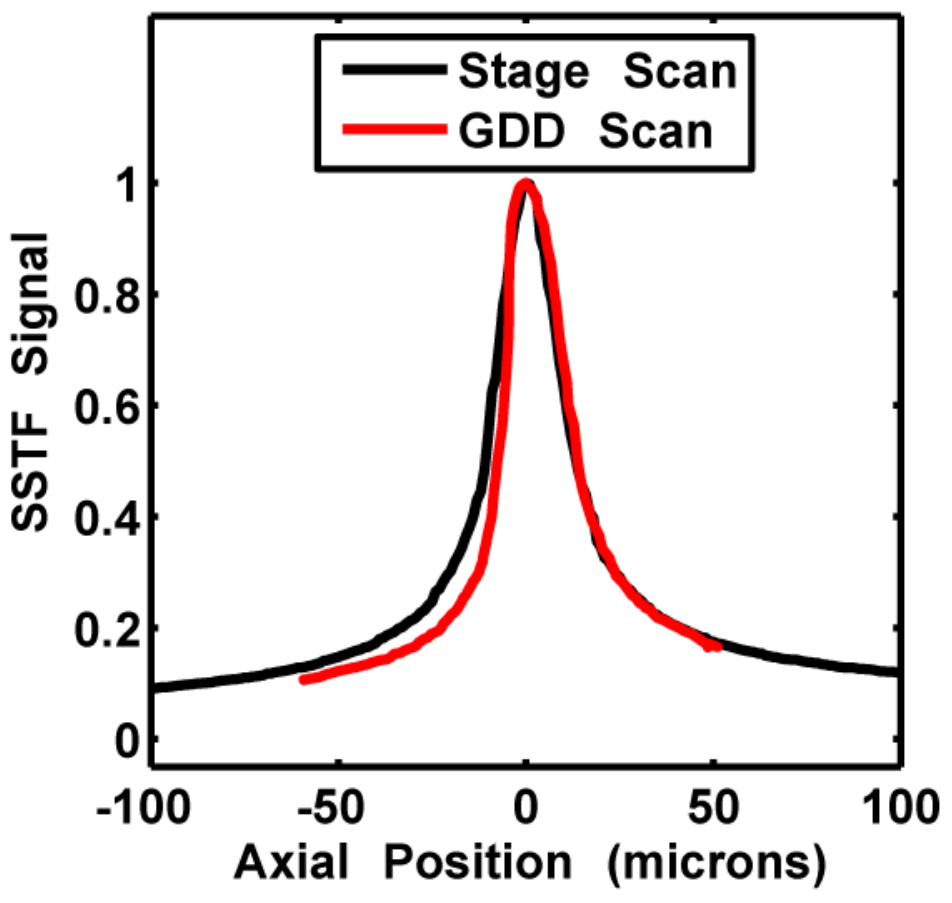

Figure 5.5: Axial scans of two-photon excited fluorescence from a thin fluorescent film by mechanically moving the sample stage (black curve) and by tuning the GDD (red curve) at $2 \mathrm{kHz}$ in a SSTF setup. 
allowing for remote scanning of the focal plane by tuning the GDD on the proximal end of the fiber. Therefore, by applying our dispersion compensation technique, remote axial scanning of the focal plane can be realized at high speed, achieving optical coherence tomography (OCT)-like axial scans in a two-photon fluorescence or SHG imaging probe.

We have demonstrated a dispersion tuning technique that utilizes a piezo bimorph mirror. It is cost-effective, high-speed, broadband, has a large range, and can tolerate high intensities. We have further demonstrated its application for high-speed axial scanning of two-photon excited fluorescence in an SSTF setup, scanning over a range of 100 microns at a rate of $2 \mathrm{kHz}$.

\section{Acknowledgments}

This research was made possible by the National Institutes of Health (NIH) (grant R21CA129648). 


\section{REFERENCES}

1. W. R. Zipfel, R. M. Williams, and W. W. Webb, "Nonlinear magic: multiphoton microscopy in the biosciences," Nature Biotechnology 21, 1368-1376 (2003).

2. V. Iyer, B. E. Losavio, and P. Saggau, "Compensation of spatial and temporal dispersion for acousto-optic multiphoton laser-scanning microscopy," Journal of Biomedical Optics 8, 471 (2003).

3. M. E. Durst, G. Zhu, and C. Xu, "Simultaneous spatial and temporal focusing in nonlinear microscopy," Optics Communications 281, 1805 (2008).

4. D. Oron, E. Tal, and Y. Silberberg, "Scanningless depth-resolved microscopy," Optics Express 13, 1468-1476 (2005).

5. M. E. Durst, G. Zhu, and C. Xu, "Simultaneous spatial and temporal focusing for axial scanning," Optics Express 14, 12243-12254 (2006).

6. H. Suchowski, D. Oron, and Y. Silberberg, "Generation of a dark nonlinear focus by spatio-temporal coherent control," Optics Communications 264, 482-487 (2006).

7. R. Du, K. Bi, S. Zeng, D. Li, S. Xue, and Q. Luo, "Analysis of fast axial scanning scheme using temporal focusing with acousto-optic deflectors," Journal of Modern Optics 56, 102 (2009).

8. R. L. Fork, O. E. Martinez, and J. P. Gordon, "Negative Dispersion Using Pairs of Prisms," Optics Letters 9, 150-152 (1984).

9. O. E. Martinez, "3000 Times Grating Compressor with Positive GroupVelocity Dispersion - Application to Fiber Compensation in 1.3-1.6 Mu-M Region," IEEE Journal of Quantum Electronics 23, 59-64 (1987).

10. E. A. Gibson, D. M. Gaudiosi, H. C. Kapteyn, R. Jimenez, S. Kane, R. Huff, 
C. Durfee, and J. Squier, "Efficient reflection grisms for pulse compression and dispersion compensation of femtosecond pulses," Opt. Lett. 31, 3365 (2006).

11. P. Tournois, "Acousto-optic programmable dispersive filter for adaptive compensation of group delay time dispersion in laser systems," Optics

Communications 140, 245-249 (1997).

12. A. M. Weiner, D. E. Leaird, J. S. Patel, and J. R. Wullert, "Programmable Femtosecond Pulse Shaping by Use of a Multielement Liquid-Crystal Phase Modulator," Optics Letters 15, 326-328 (1990).

13. E. Zeek, K. Maginnis, S. Backus, U. Russek, M. Murnane, G. Mourou, H. Kapteyn, and G. Vdovin, "Pulse compression by use of deformable mirrors," Optics Letters 24, 493-495 (1999).

14. C. Radzewicz, P. Wasylczyk, W. Wasilewski, and J. S. Krasiski, "Piezo-driven deformable mirror for femtosecond pulse shaping," Opt. Lett. 29, 179 (2004).

15. M. E. Durst, D. Kobat, and C. Xu, "Tunable dispersion compensation by a rotating cylindrical lens," Opt. Lett. 34, 1197 (2009).

16. Y. Ozeki, G. Omura, and K. Itoh, "Broadband group delay dispersion compensation for a microscope objective lens with a specially-designed mechanical deformable mirror," Optics Express 16, 2778-2783 (2008).

17. Q. Li, M. Lovell, J. Mei, and W. Clark, "A Study of Displacement Distribution in a Piezoelectric Heterogeneous Bimorph," Journal of Mechanical Design 126, 757762 (2004).

18. M. R. Steel, F. Harrison, and P. G. Harper, "The piezoelectric bimorph: An experimental and theoretical study of its quasistatic response," Journal of Physics D: Applied Physics, 979 (1978).

19. P. Wnuk, C. Radzewicz, and J. Krasinski, "Bimorph piezo deformable mirror for femtosecond pulse shaping," Opt. Express 13, 4159 (2005). 
20. "Introduction to Piezo Transducers," (Piezo Systems, Inc., 2007), http://www.piezo.com/tech2intropiezotrans.html, Accessed June 24, 2009. 


\title{
APPENDIX A
}

\section{ENHANCED AXIAL CONFINEMENT OF SUM FREQUENCY GENERATION IN}

\section{A TEMPORAL FOCUSING SETUP ${ }^{6}$}

\begin{abstract}
We demonstrate enhanced axial confinement in a temporal focusing setup with a shaped spectrum and a narrow emission filter, achieving an order of magnitude reduction of the out-of-focus background when compared to conventional point-scanning two-photon microscopy. By rejecting the background in the optical domain, our technique circumvents the noise problems common in other background subtraction techniques.
\end{abstract}

\section{A.1. Introduction}

Multiphoton fluorescence and second harmonic generation (SHG) microscopy (MPM) are powerful tools for imaging biological samples due to their ability to perform optical sectioning [1]. Several methods have been proposed to enhance the axial confinement by suppressing the background due to out-of-focus excitation. Differential aberration imaging measures the background by intentionally eliminating the two-photon excited fluorescence (TPEF) at the focus through added aberrations in the system [2]. The background can then be subtracted from an unaberrated image in order to enhance the signal-to-background ratio (SBR). Oron et al. proposed using coherent control to enhance the confinement of simultaneous spatial and temporal focusing (SSTF) [3], in which the frequencies of an ultrashort pulse are spatially separated by a grating and recombined at the focus $[4,5]$. Spectral phase shaping can then selectively turn off the TPEF from the focus in order to measure the out-of-focus background. While both background subtraction techniques demonstrate improved

\footnotetext{
${ }^{6}$ The contents of this appendix have been reproduced from Optics Letters, 34, No. 12, 1786 (2009).
} 
axial confinement, the subtraction operation in fact increases the background noise contribution. Therefore, background subtraction techniques decrease the signal-tonoise ratio (SNR), which is ultimately the limiting factor for imaging performance.

Two-color two-photon (2C2P) microscopy spatially separates the two excitation wavelengths such that no signal corresponding to the sum-frequency generation (SFG) can be generated outside of the focus [6-8]. By filtering the background photons optically, both the background and its associated noise can be suppressed. Typically, 2C2P microscopy requires two synchronized pulse sources, such as a Ti:Sapphire laser pumping an optical parametric oscillator (OPO). The pulses at the two wavelengths must be spatially and temporally overlapped in order to observe a SFG effect.

\section{A.2. Theory}

We propose a technique which combines SSTF with the SFG produced by 2C2P microscopy using a single laser source. In SSTF, an ultra-short pulse is incident on a grating, which is then imaged onto the sample in a 4-f system. Because the grating separates the spectral components of the pulse in space, the two "colors" for a $2 \mathrm{C} 2 \mathrm{P}$ setup can be generated by simply blocking the center of the spectrum at the input focal plane of the objective (see Figure A.1). A theoretical understanding of the SFG process can be found by a coherent summation of the SFG fields due to all the dipole emitters excited within the sample [9-11]. Due to the coherent nature of SFG, directionality of emission is highly sample dependent, and in general closed-form analytical solutions cannot be attained. A numerical solution is available by following the rigorous treatment of harmonic imaging with conventional SSTF [12]. Intuitively, the outer spectral components of our spectrally-shaped SSTF beam will be overlapped spatially only at the focus, producing a SFG signal at half the center wavelength. By 


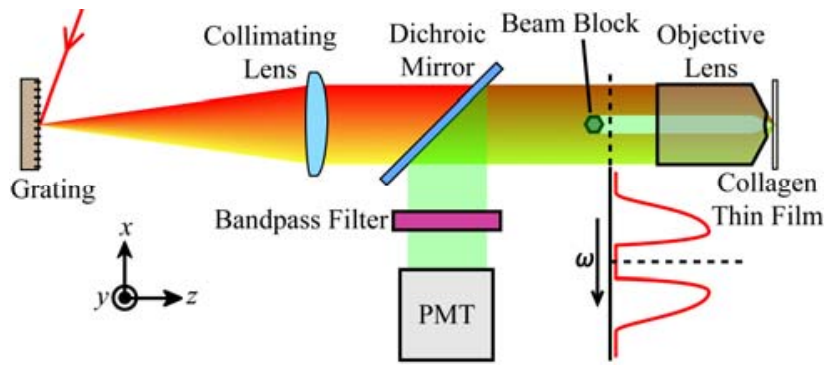

Figure A.1. Experimental SFG SSTF setup. 
spectrally filtering the collected signal, second harmonic generation by the two beams outside the overlapping region is rejected, and only the signal from the common focus is detected, achieving an improved axial confinement.

We investigate axial confinement for the two imaging modalities of SSTF: wide field and line scanning. In the SSTF wide-field case, the beam shape at the input focal plane of the objective is a thin line, where the width in the $x$-direction is the spread of the monochromatic components due to the grating, and the height in the $y$ direction is the individual monochromatic beam size. Unlike conventional imaging, wide-field imaging with SSTF allows for optical sectioning due to the evolution of the temporal pulse width within the sample, yielding the same axial confinement as conventional line-scanning imaging [13]. In SSTF line scanning, the beam at the back aperture is expanded in the y-dimension, yielding spatial focusing in the $y$-dimension and temporal focusing in $x$. Thus, line-scanning SSTF has the same axial confinement as point-scanning MPM [13].

\section{A.3. Experiment}

The experimental SFG SSTF setup is shown in Figure 1. We use a modelocked Ti:Sapphire laser (Spectra-Physics Tsunami) centered at $744 \mathrm{~nm}$ with $14 \mathrm{~nm}$ of bandwidth (FWHM). A ruled diffraction grating with a 1200 lines $/ \mathrm{mm}$ groove density separates the beam into its monochromatic components, which are then collimated by a $40 \mathrm{~cm}$ focal-length lens and focused by an objective lens (Zeiss Fluar 20x/0.75 NA). We spectrally shape the beam by placing a metal rod ( $1 / 8$ " hex key) at the input focal plane of the objective lens, blocking the center $7 \mathrm{~nm}$ (FWHM) of bandwidth. Note that the lateral spot size at the focus remains unchanged because this is determined by the magnification of the individual monochromatic beams. Our sample is a thin film $(\sim 3 \mu \mathrm{m})$ of inhomogeneous Type I collagen on a \#1 cover glass. The collagen film 
sample is prepared by drying a gel of Bovine I collagen (Invitrogen). Loosely arranged Type I collagen has been shown to produce a significant amount of SHG in the backward direction $[14,15]$, facilitating imaging in the epidirection.

To observe the signal spectrum as a function of position we use an optical fiber to collect the SFG signal into a spectrometer (Horiba SPEX 270M). We use an excitation spectrum centered at $800 \mathrm{~nm}$ due to the sensitivity of the spectrometer. Figure A.2 shows the resulting SFG spectrum versus distance from the focus. The two outer peaks correspond to the SHG of the two excitation beams individually. The large central peak corresponds to the SFG of the two beams together. Note that the outer SHG peaks extend much further axially than the central SFG peak. Axial confinement is measured by scanning the collagen film through the focus on a translation stage (Figure A.1). A narrow bandwidth (1 nm FWHM) emission filter (Semrock) centered at $372 \mathrm{~nm}$ selects the SFG from the focal region and rejects the SHG background. The signal is detected in the epi-direction by a photomultiplier tube (PMT, Hamamatsu HC125-02). We perform these axial scans in both the wide-field and line-scanning SSTF configurations (blue squares in Figure A.3(a) and (b), respectively). We also compare the SSTF axial profile to that of a conventional SHG experiment with the same objective lens (red triangles). Without spectral shaping, the SFG SSTF curves follow closely their corresponding SHG excitation profiles. With spectral shaping and filtering, the confinement improves in both wide-field and linescanning SSTF, each to an order of magnitude improvement 30 microns from the focus (blue circles). Observe that the confinement is worse with the hex key but without the optical filter (green circles). This demonstrates that our effective pupil shaping is not the source of the enhanced confinement. We note that in the wide-field SSTF configuration (Figure A.3(a)), the background suppression technique 


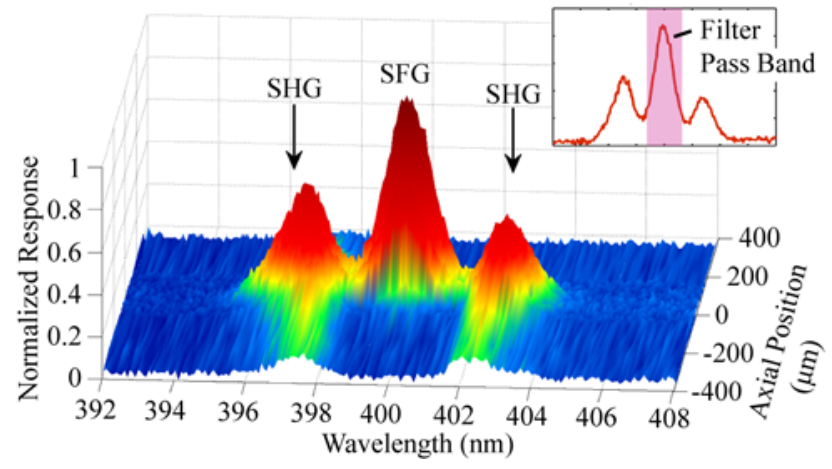

Figure A.2. Measured spectrum as a function of axial position. Inset: desired filter bandwidth to detect only SFG contribution of the signal. 


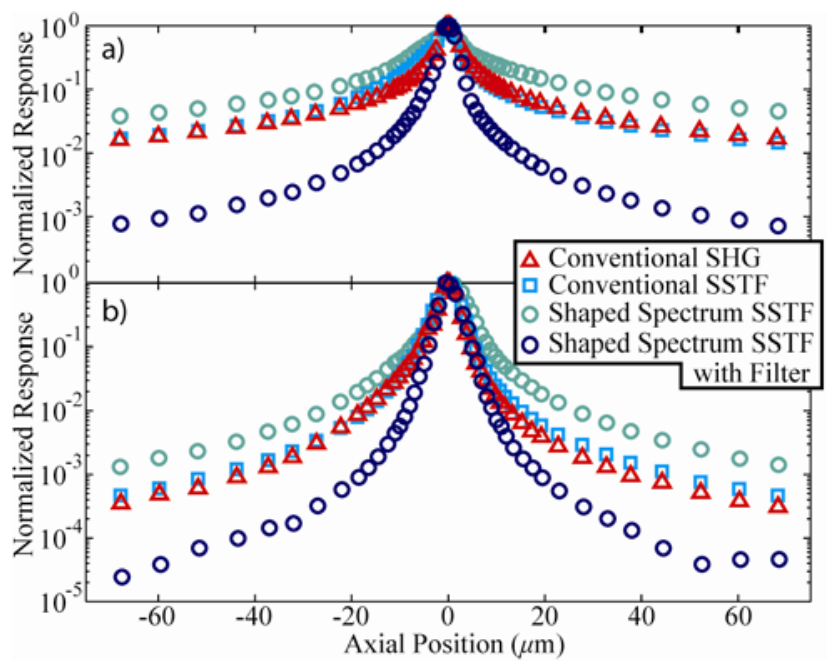

Figure A.3. Measured thin film axial scans in the (a) wide-field and (b) line-scanning SSTF configurations, with corresponding (a) line and (b) point SHG scans (log scale). 
significantly enhances the axial confinement and achieves performance similar to a conventional point-scanning system. In the line-scanning SSTF configuration, our technique significantly out-performs a conventional point-scanning system.

To demonstrate the utility of the enhanced axial confinement in the SFG SSTF technique, we prepare two thin collagen films separated by 45 microns. Keeping the narrow bandwidth filter in place, wide-field SSTF axial scans with and without spectral shaping are taken by sweeping the sample through the focus (Figure A.4). Although the films are far apart (approximately ten times the axial FWHM), the poor axial confinement of standard wide-field SSTF yields a background level of $7 \%$ in the overlapped region between the peaks. With a shaped spectrum, however, the two films are more clearly defined, and the background is reduced to $0.6 \%$ between the peaks. By simply adding a metal rod into the beam path, the axial confinement for wide-field SSTF is improved by an order of magnitude, achieving performance that is comparable to conventional point-scanning TPEF microscopy.

\section{A.4. Discussion}

There are many advantages to performing SFG in a SSTF setup. 2C2P microscopy typically requires two synchronized laser sources, whereas this technique requires only one. This requirement also eliminates the need for complicated delay lines and other sensitive alignment techniques in order to have the two "colors" spatially and temporally overlap at the focus. In addition, SSTF has the advantage of being able to scan the temporal focus axially by tuning the group-delay dispersion (GDD) [13]. Therefore, this SFG technique combined with SSTF can yield a remote scanning fiber probe with enhanced axial confinement and no moving parts at the distal end. 


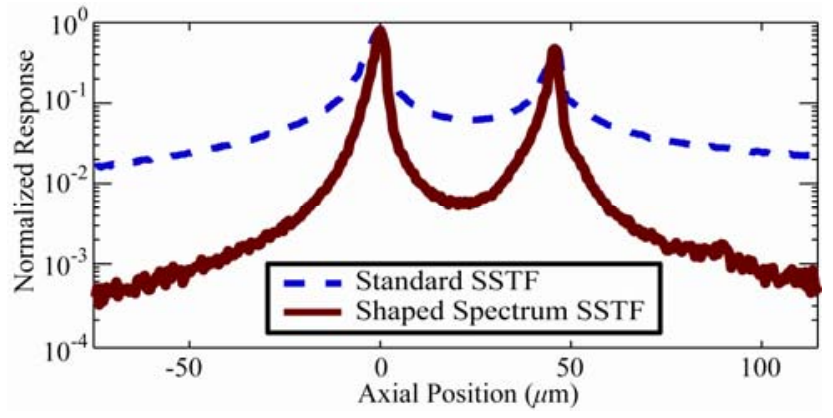

Figure A.4. Measured axial response of two collagen films placed 45 microns apart with (solid line) and without (dashed line) spectral shaping (log scale). 
Because images in a wide-field or line-scanning SSTF setup must be captured with a camera, this technique has limited use when imaging deep into scattering tissue. At smaller depths or in non-scattering situations, however, wide-field SSTF can be valuable [16]. By incorporating our SFG SSTF technique, an order of magnitude suppression of the out-of-focus background can be obtained at a distance as small as 30 microns away from the focus. Such a reduction in background is particularly valuable for wide-field SSTF, where the poor axial confinement is a major concern. A potential drawback of the proposed technique is that the distorted temporal profile of the pulse and the power loss due to the partial obstruction of the excitation beam make the SFG SSTF process inefficient. While sufficient signal can be generated by increasing the excitation power, there are more efficient approaches to shaping the spectrum of the excitation source. For example, it is well known that self-phase modulation can efficiently generate a spectrum with a central minimum without losing power or distorting the temporal intensity profile.

\section{A.5. Conclusion}

We have demonstrated enhanced axial confinement by combining SFG with a SSTF setup, achieving more than an order of magnitude reduction of the out-of-focus background when compared to conventional TPEF microscopy. By rejecting the background in the optical domain using a narrow-band optical filter (i.e., before the background photons reach the detector), our technique does not suffer noise problems common in other background subtraction techniques.

This research was made possible by Grant Number R21CA129648 from the National Institutes of Health. 


\section{REFERENCES}

1. W. R. Zipfel, R. M. Williams, and W. W. Webb, "Nonlinear magic: multiphoton microscopy in the biosciences," Nature Biotechnology 21, 1368-1376 (2003).

2. A. Leray, and J. Mertz, "Rejection of two-photon fluorescence background in thick tissue by differential aberration imaging," Opt. Express 14, 10573 (2006).

3. D. Oron, and Y. Silberberg, "Spatiotemporal coherent control using shaped, temporally focused pulses," Optics Express 13, 9903-9908 (2005).

4. G. Zhu, J. van Howe, M. Durst, W. Zipfel, and C. Xu, "Simultaneous spatial and temporal focusing of femtosecond pulses," Optics Express 13, 2153-2159 (2005). 5. D. Oron, E. Tal, and Y. Silberberg, "Scanningless depth-resolved microscopy," Optics Express 13, 1468-1476 (2005).

6. M. O. Cambaliza, and C. Saloma, "Advantages of two-color excitation fluorescence microscopy with two confocal excitation beams," Optics Communications 184, 35 (2000).

7. D. Kobat, G. Zhu, and C. Xu, "Background Reduction with Two-Color TwoBeam Multiphoton Excitation," in Biomedical Optics(Optical Society of America, 2008), p. BMF6.

8. S. Lindek, and E. H. K. Stelzer, "Resolution improvement by nonconfocal theta microscopy," Opt. Lett. 24, 1507 (1999).

9. L. Moreaux, O. Sandre, and J. Mertz, "Membrane imaging by secondharmonic generation microscopy," J. Opt. Soc. Am. B 17, 1694 (2000).

10. J. Mertz, and L. Moreaux, "Second-harmonic generation by focused excitation of inhomogeneously distributed scatterers," Optics Communications 196, 330 (2001). 
11. R. M. Williams, W. R. Zipfel, and W. W. Webb, "Interpreting secondharmonic generation images of collagen I fibrils," Biophysical Journal 88, 1377-1386 (2005).

12. D. Oron, and Y. Silberberg, "Harmonic generation with temporally focused ultrashort pulses," Journal of the Optical Society of America B-Optical Physics 22, 2660-2663 (2005).

13. M. E. Durst, G. Zhu, and C. Xu, "Simultaneous spatial and temporal focusing in nonlinear microscopy," Optics Communications 281, 1805 (2008).

14. W. R. Zipfel, R. M. Williams, R. Christie, A. Y. Nikitin, B. T. Hyman, and W. W. Webb, "Live tissue intrinsic emission microscopy using multiphoton-excited native fluorescence and second harmonic generation," Proceedings of the National Academy of Sciences of the United States of America 100, 7075-7080 (2003).

15. S.-W. Chu, S.-P. Tai, T.-M. Liu, C.-K. Sun, and C.-H. Lin, "Selective imaging in second-harmonic-generation microscopy with anisotropic radiation," Journal of Biomedical Optics 14, 3 (2009).

16. A. Vaziri, J. Tang, H. Shroff, and C. V. Shank, "Multilayer three-dimensional super resolution imaging of thick biological samples," Proceedings of the National Academy of Sciences 105, 20221-20226 (2008). 


\section{APPENDIX B}

\section{DETAILED FORMALISM FOR AXIAL SCANNING WITH GDD TUNING}

The purpose of this appendix is to derive an expression for the intensity $I$ of the field at the focal volume as a function of the axial position $z$. Using the paraxial approximation, the Fresnel diffraction equation in one dimension [1] is:

$$
U_{1}(x)=\frac{e^{i k f}}{\sqrt{i \lambda f}} \int_{-\infty}^{\infty} U_{0}(\xi) e^{i \frac{k}{2 f}(x-\xi)^{2}} d \xi
$$

We propagate the field to the objective lens with the Fresnel diffraction equation (Eq. (B.1)) by substituting $A_{1}(x, \omega)$ from Eq. (2.1) into $U_{0}(x)$ in Eq. (B.1). The field just before the lens is therefore:

$$
\begin{gathered}
A_{2}(x, \omega)=A_{0} \frac{e^{i k f}}{\sqrt{i \lambda f}} e^{-\frac{\omega^{2}}{\Omega^{2}}} e^{i \beta \omega^{2}} \int_{-\infty}^{\infty} e^{\frac{-(\xi-\alpha \omega)^{2}}{s^{2}}} e^{i \frac{k}{2 f}(x-\xi)^{2}} d \xi \\
=A_{0} e^{i k f} e^{-\frac{\omega^{2}}{\Omega^{2}}} e^{i \beta \omega^{2}} \frac{1}{\left(1+i \frac{2 f}{k s^{2}}\right)^{\frac{1}{2}}} e^{-\frac{k(x-\alpha \omega)^{2}}{2 i f+k s^{2}}}
\end{gathered}
$$

After adding the quadratic phase due to the lens, the field becomes:

$$
A_{3}(x, \omega)=A_{0} e^{i k f} e^{-\frac{\omega^{2}}{\Omega^{2}}} e^{i \beta \omega^{2}} \frac{1}{\left(1+i \frac{2 f}{k s^{2}}\right)^{\frac{1}{2}}} e^{-\frac{k(x-\alpha \omega)^{2}}{2 i f+k s^{2}}} e^{-i \frac{k}{2 f} x^{2}}
$$

By propagating the field a distance $z$ from the lens with the Fresnel diffraction equation (Eq. (B.1)), the field in the focal volume can be expressed as:

$$
\begin{gathered}
A_{4}(x, z, \omega)=A_{0} e^{i k(z+f)} e^{-\frac{\omega^{2}}{\Omega^{2}}} e^{i \beta \omega^{2}} \frac{1}{\left(1+i \frac{2 f}{k s^{2}}\right)^{\frac{1}{2}}} \frac{1}{\left(1+i \frac{2 z}{k s_{1}^{2}}\right)^{\frac{1}{2}}} \cdot \ldots \\
\text { where } s_{1}^{2}=\frac{4 f^{2}}{k^{2} s^{2}}-i \frac{2 f}{k} \text { and } s_{2}^{2}=\frac{4 f^{2}}{k^{2} s^{2}}+i \frac{2(z-f)}{k} .
\end{gathered}
$$


To simplify calculations, we assume that the wavevector $k$ for each frequency is approximately $k_{0}$, the wavevector of the center wavelength of the pulse. Fourier transforming $A_{4}(\omega, x, z)$ back into the time domain, the field distribution at the focal volume is:

$$
\begin{aligned}
& A_{5}(x, z, t)=\int_{-\infty}^{\infty} A_{4}(x, z, \omega) \cdot e^{-i \omega t} d \omega \\
& =\kappa \cdot e^{-\frac{x^{2}}{s_{2}^{2}}} e^{-\frac{\Omega^{2}}{4(1+\chi)}(t+\gamma x)^{2}}
\end{aligned}
$$

where

$$
\begin{gathered}
\kappa=A_{0} \Omega \sqrt{\frac{i \pi f}{z_{M}}} \frac{1}{\left[\beta \Omega^{2} \frac{f-z}{z_{M}}-1+i\left(\frac{f-z}{z_{R}}+\beta \Omega^{2}\right)\right]^{\frac{1}{2}}}, \\
\gamma=\frac{k_{0} \alpha / f}{1+i(z-f) / z_{M}}, \\
\chi=\frac{i(z-f) / z_{B}}{1+i(z-f) / z_{M}}-i \beta \Omega^{2}, \\
z_{M}=\frac{2 f^{2}}{k_{0} s^{2}}, z_{R}=\frac{2 f^{2} / k_{0}}{s^{2}+\alpha^{2} \Omega^{2}}, \text { and } z_{B}=\frac{2 f^{2}}{k_{0} \alpha^{2} \Omega^{2}} .
\end{gathered}
$$

The intensity can then be expressed as:

$$
I(x, z, t)=\left|A_{5}\right|^{2}=\left|\kappa e^{-\frac{x^{2}}{s_{2}^{2}}} e^{-\frac{\Omega^{2}}{4(1+\chi)}(t+\gamma x)^{2}}\right|^{2}
$$

The pulse width at the temporal focus can be obtained by substituting $z=f+\beta \Omega^{2} z_{R}$ and $x=0$ into Eq. (B.6). The pulse width $\tau$ at the temporal focus is:

$$
\tau=\tau_{0}\left[1+\left(\beta \Omega^{2}\right)^{2} \frac{z_{R}}{z_{M}}\right]^{\frac{1}{2}}
$$

where $\tau_{0}=\sqrt{2} / \Omega$ is the transform-limited pulse width. At the maximum shift defined by Eq. (2.6), the pulse width broadens to $\tau=\sqrt{2} \tau_{0}$. Similarly, the lateral spot size at the temporal focus can be calculated by substituting $z=f+\beta \Omega^{2} z_{R}$ and $t=0$ into Eq. 
(B.6). This yields the lateral spot size at the temporal focus:

$$
s_{3}=s_{3}^{(0)}\left[1+\left(\beta \Omega^{2}\right)^{2} \frac{z_{R}}{z_{M}}\right]^{\frac{1}{2}}
$$

where $s_{3}^{(0)}$ is the lateral spot size when GDD is zero. Therefore, at the onset of broadening (Eq. (2.6)), the lateral spot size stretches to $s_{3}=\sqrt{2} s_{3}^{(0)}$. 


\section{REFERENCES}

1. J. Goodman, Introduction to Fourier Optics (Roberts \& Company, Englewood, Colorado, 2005). 


\section{APPENDIX C \\ EFFECTS OF REFRACTIVE-INDEX MISMATCH AND SCATTERING ON SIMULTANEOUS SPATIAL AND TEMPORAL FOCUSING ${ }^{7}$}

\section{C.1. Introduction}

Simultaneous spatial and temporal focusing (SSTF) improves the axial excitation confinement of line-scanning and wide-field imaging when combined with multiphoton microscopy $[1,2]$. SSTF provides axial confinement by spatially separating the frequency components of a pulse with a grating and recombining them with an objective lens, creating a temporal focus. The unique design of SSTF offers many advantages, including fast 3D imaging, automatic dispersion compensation, and remote axial scanning [1-4].

In SSTF, the pulse width at the temporal focus has been shown to be transform limited in a non-scattering sample [2]. With the aberrations induced by refractiveindex mismatch and sample scattering, however, both the phase and amplitude of the frequency components of the pulse may not recover their original values at the focus. For example, in a scattering sample, the individual frequency components of the excitation pulse travel different distances through the scattering medium to the focus, and the marginal rays are attenuated more than those at the center of the beam. Therefore, the spectrum of the pulse narrows, and the pulse width at the temporal focus broadens. In this appendix, we investigate the pulse-broadening effects of refractive-index mismatch and scattering on SSTF.

\footnotetext{
${ }^{7}$ The contents of this appendix have been reproduced from Frontiers in Optics, 2007.
} 


\section{C.2. Theory}

In a scattering sample, rays incident at different angles see varying amounts of attenuation based on the path length $(L)$ traveled between the surface of the scattering medium and the focus (Figure 1):

$$
L(z, \omega)=\left[z-\left(f-z_{0}\right)\right] \sqrt{1+\left(\frac{\alpha \omega}{f}\right)^{2}}
$$

For $\alpha \omega / f \ll 1, L$ can be approximated as:

$$
L(z, \omega)=\left[z-\left(f-z_{0}\right)\right]\left[1+\frac{1}{2}\left(\frac{\alpha \omega}{f}\right)^{2}\right]
$$

We follow the derivation for wide-field SSTF (Appendix B), but we now add an exponential decay representing the power loss due to scattering:

$$
A^{\prime}(x, y, z, \omega)=A(x, y, z, \omega) e^{-\frac{L(z, \omega)}{2 l_{s}}}
$$

Our field can now be written as:

$$
\begin{aligned}
A(x, y, z, \omega)= & A_{0} e^{i k(z+f)} e^{-\frac{\omega^{2}}{\Omega^{2}}} e^{i \beta \omega^{2}} \frac{1}{\left(1+i \frac{2 f}{k s^{2}}\right)^{\frac{1}{2}}} \frac{1}{\left(1+i \frac{2 z}{k s_{1}^{2}}\right)^{\frac{1}{2}} \cdot \cdots} \\
& \ldots \cdot e^{-\frac{\left(x-\frac{(f-z)}{f} \alpha \omega\right)^{2}}{s_{2}^{2}}} e^{-i \frac{k \alpha \omega x}{f}} e^{-i \frac{k \alpha^{2} \omega^{2}(z-f)}{2 f^{2}}} \cdot e^{-\frac{\left[z-\left(f-z_{0}\right)\right]\left[1+\frac{1}{2}\left(\frac{\alpha \omega}{f}\right)^{2}\right]}{2 l_{s}}}
\end{aligned}
$$

Observe that Eq. (C.4) can be reduced to its old form (without scattering, Appendix B) by making a simple substitution:

$$
\begin{aligned}
& \frac{1}{\Omega^{\prime 2}(z)}=\frac{1}{\Omega^{2}}+\frac{\alpha^{2}}{4 f^{2} l_{s}}\left(z-f+z_{0}\right) \\
& \Rightarrow \Omega^{\prime 2}(z)=\Omega^{2} \frac{1}{1+\frac{\alpha^{2} \Omega^{2}}{4 f^{2} l_{s}}\left(z-f+z_{0}\right)}
\end{aligned}
$$

Because the effective bandwidth is now narrowed, the transform-limited pulse width at the temporal focus will no longer be recovered. The pulse width as a function of position can be written as: 


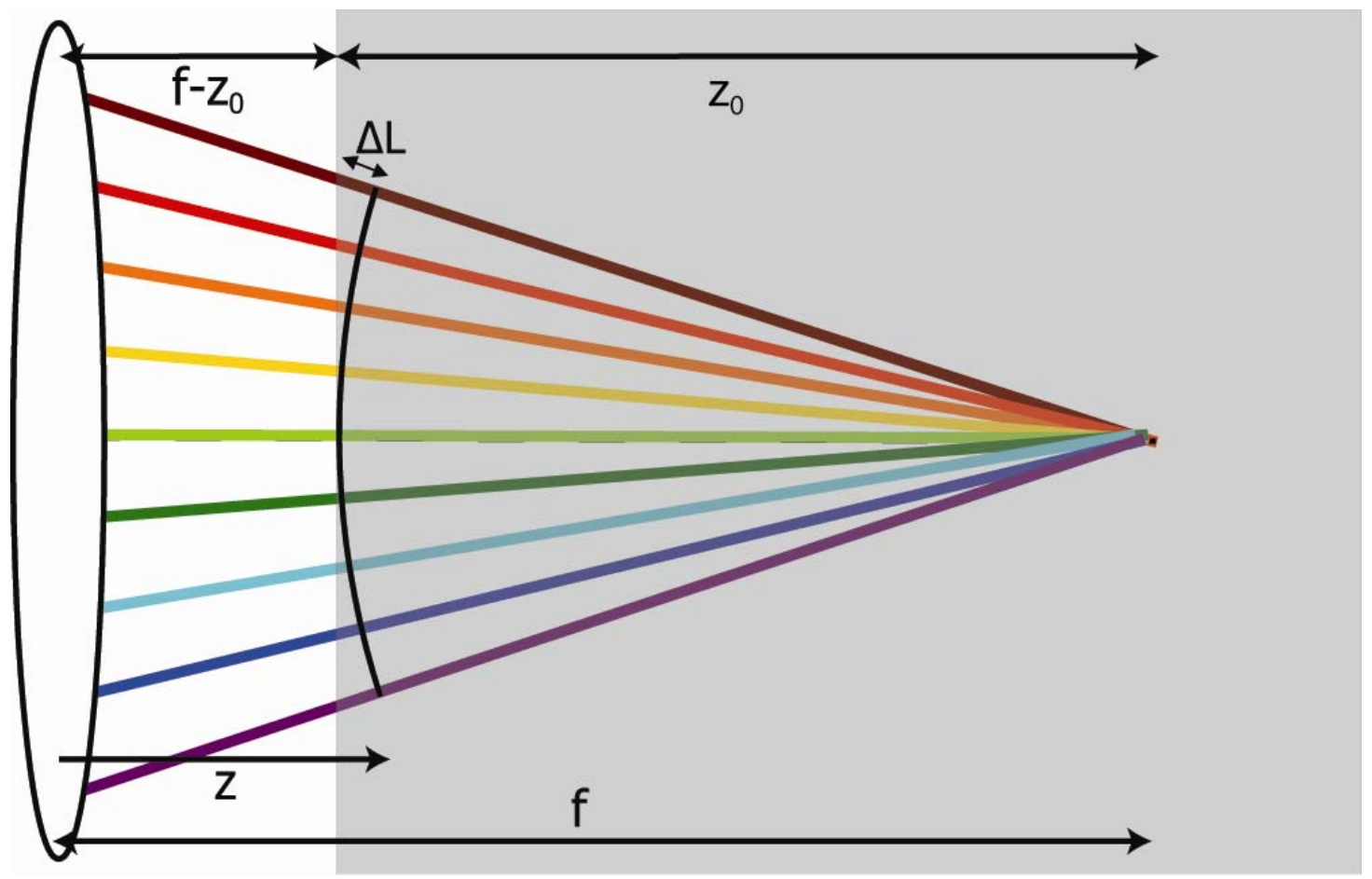

Figure C.1: Focal volume of a typical SSTF setup. Gray area represents onset of scattering medium at a distance $\mathrm{z}_{0}$ from the objective. Black arc shows a radius of a circle centered at the focus, such that marginal rays travel a distance $\Delta L$ longer than the center ray. 


$$
\tau_{\text {wide }}=\left[2\left(\frac{4 f^{2} l_{s}+\alpha^{2}\left(z-f+z_{0}\right) \Omega^{2}}{4 f^{2} l_{s} \Omega^{2}}\right)\left(1+\frac{z_{M}}{z_{B}} \frac{(z-f)^{2}}{(z-f)^{2}+z_{M} z_{R}}\right)\right]^{\frac{1}{2}}
$$

At the focal plane $(\mathrm{z}=\mathrm{f})$, the pulse width can be rewritten as:

$$
\tau_{\text {wide }}=\tau_{0}\left[1+\frac{\alpha^{2} \Omega^{2} z_{0}}{4 f^{2} l_{s}}\right]^{\frac{1}{2}}
$$

Figure C.2 shows how a 100 fs pulse at five scattering lengths will broaden as a function of the numerical aperture (NA) of the objective lens. For NA=0.45, $\alpha \Omega / f \sim 0.5$, so the pulse width is reduced to:

$$
\tau_{\text {wide }}=\tau_{0}\left[1+\frac{z_{0}}{16 l_{s}}\right]^{\frac{1}{2}}
$$

For typical values of $z_{0}=1 \mathrm{~mm}$ and $l_{\mathrm{s}}=200$ microns, the pulse width broadens to:

$$
\tau_{\text {wide }}=\tau_{0} \cdot 1.15
$$

For a highly scattering specimen with $l_{\mathrm{s}}=20$ microns, the pulse width broadens to:

$$
\tau_{\text {wide }}=\tau_{0} \cdot 2.03
$$

\section{C.3. Experiment}

The experimental setup is shown in Figure C.3. We use a mode-locked Ti:Sapphire laser (Spectra-Physics Tsunami) centered at $775 \mathrm{~nm}$ with $100 \mathrm{fs}$ pulses (assuming a sech shape) at an $80 \mathrm{MHz}$ repetition rate. To measure the pulse width at the sample, we use an interferometric second-order autocorrelator which uses TPEF from a thin Rhodamine film as the nonlinear element. A ruled diffraction grating with 1200 lines/mm separates the beam into its monochromatic components, which are then collimated by a spherical lens and refocused by an objective. To collect the fluorescence signal, a dichroic mirror reflects the TPEF signal onto a PMT (Hamamatsu HC125-02) through filters that further remove the excitation light. 


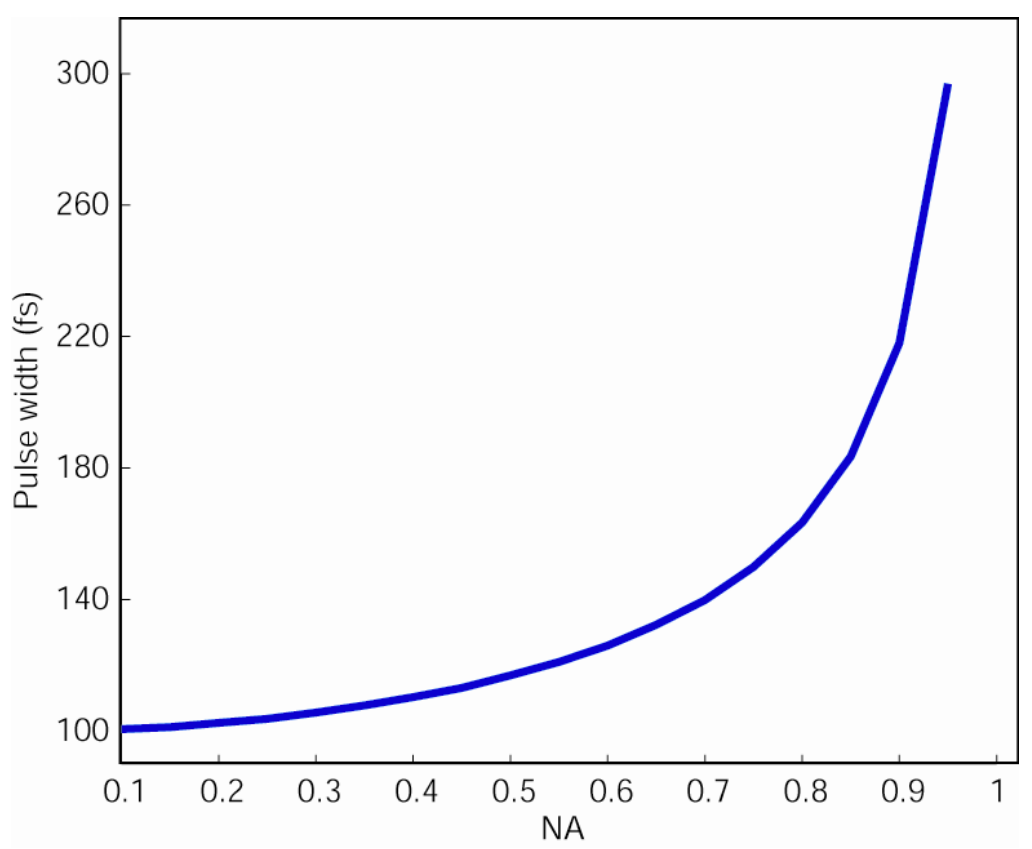

Figure C.2: Plot of pulse width at the temporal focus versus NA of the objective at a depth of five scattering lengths. 


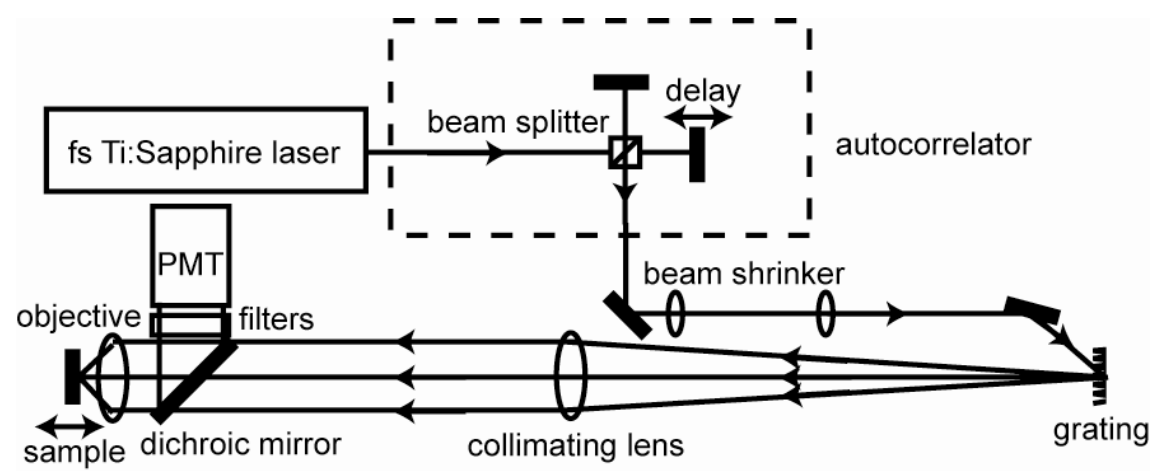

Figure C.3: Experimental setup. BS: beam splitter. CL: collimating lens. DM: dichroic mirror. OBJ: objective lens. 
The sample consists of $1 \mu \mathrm{m}$ polystyrene beads suspended in Agarose gel with a scattering length of $150 \mu \mathrm{m}$. A thin film of Rhodamine B is located at the bottom surface of the gel. The air objectives we used are a Zeiss Plan-Apochromat 10x/0.45, a Zeiss Fluar 20x/0.75, and a Zeiss IR-Epiplan 100x/0.9 objective. Second-order autocorrelation traces are obtained while focusing onto the back film at a depth of one scattering length. First, we measure the pulse width below the Agarose gel both with and without scatterers for the air objectives. As shown in Figure C.4, the pulse width broadens as the NA increases for samples both with and without scatterers when air objectives are used. We then use two different water immersion objectives (Olympus UMPlanFl 20x/0.5 W and an Olympus XLUMPlanF1 20x/0.95 W) to match the refractive index of the sample without scatterers. When the refractive index is matched, the pulse width at the temporal focus remains constant as a function of NA.

\section{C.4. Discussion and Conclusion}

Because the pulse width remains constant with index-matched objectives, we conclude that the pulse width broadening that occurs when no scatterers are present is due to aberrations caused by the refractive-index mismatch. The pulse broadening with scatterers present is due to both aberrations and the effect of scattering on SSTF. It may be possible to correct for this pulse-width broadening effect by countering the NA-dependent attenuation with some form of pre-compensation. This can be accomplished with amplitude modulation of the pulse spectrum with either a spatial light modulator or a simple transparency mask.

This work was made possible by Grant Number 1R21CA115472-01 from the National Cancer Institute, National Institutes of Health. 


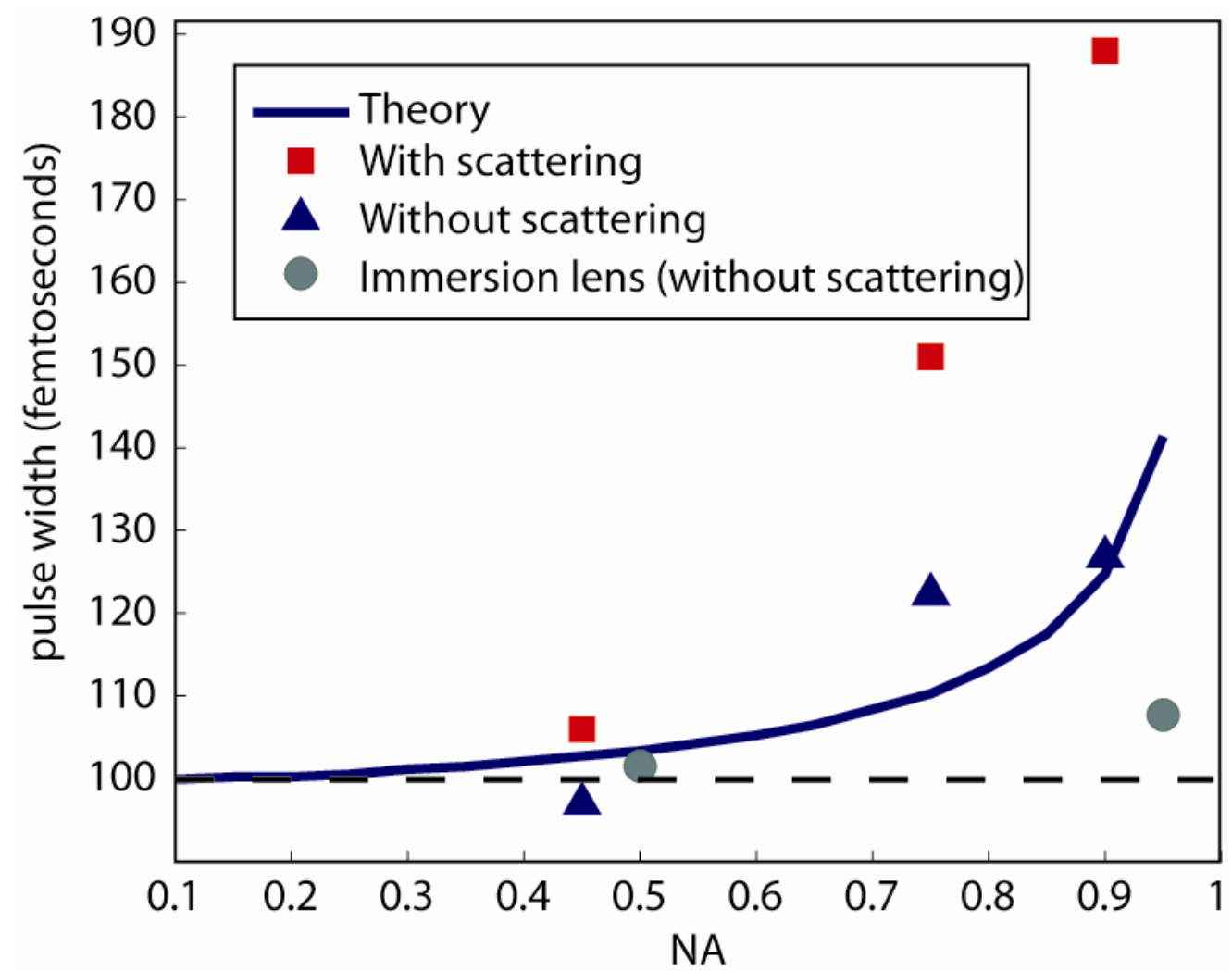

Figure C.4 Measured data of the pulse width at the temporal focus versus the numerical aperture (NA) of the objective lens at a depth of one scattering length. The squares represent measured data with a scattering sample using air objectives. The triangles are data for air objectives without scatterers in the gel. The circles are data for water immersion objectives without scatterers, showing that the pulse width indeed remains constant when there is no refractive-index mismatch. The solid curve is the theory for a sample with scatterers and no mismatch based on a Gaussian pulse. The dashed line represents the original pulse width. 


\section{REFERENCES}

1. D. Oron, E. Tal, and Y. Silberberg, "Scanningless depth-resolved microscopy," Optics Express 13, 1468-1476 (2005).

2. G. Zhu, J. van Howe, M. Durst, W. Zipfel, and C. Xu, "Simultaneous spatial and temporal focusing of femtosecond pulses," Optics Express 13, 2153-2159 (2005).

3. H. Suchowski, D. Oron, and Y. Silberberg, "Generation of a dark nonlinear focus by spatio-temporal coherent control," Optics Communications 264, 482-487 (2006).

4. M. E. Durst, G. Zhu, and C. Xu, "Simultaneous spatial and temporal focusing for axial scanning," Optics Express 14, 12243-12254 (2006). 
APPENDIX D

DETAILED COMPARISON OF WIDE-FIELD IMAGING WITH

CONVENTIONAL TWO-PHOTON AND SSTF

\section{D.1. Conventional wide-field two-photon imaging}

The total intensity of our electromagnetic wave can be written as

$I(x, y, z, t)=S(x, y, z) I_{0}(t)$. For a simple Gaussian beam, $\mathrm{I}_{0}(\mathrm{t})$ can be written as:

$$
I_{0}(t)=\frac{2 P(t)}{\pi w_{0}^{2}}=\frac{2}{\pi w_{0}^{2}}\left|A_{0}\right|^{2} e^{-\frac{t^{2}}{\tau^{2}}}
$$

The spatial component of the intensity can be written as:

$$
S(\mathbf{r})=\frac{w_{0}^{2}}{w^{2}(z)} e^{-\frac{2 \rho^{2}}{w^{2}(z)}}
$$

where

$$
w^{2}(z)=w_{0}^{2}\left[1+\left(\frac{z}{z_{R}}\right)^{2}\right]
$$

and

$$
z_{R}=\frac{n_{0} \pi w_{0}^{2}}{\lambda}
$$

Therefore, the total intensity is:

$$
I(x, y, z, t)=\frac{2\left|A_{0}\right|^{2} e^{-\frac{t^{2}}{\tau^{2}}}}{\pi w^{2}(z)} e^{-\frac{2 \rho^{2}}{w^{2}(z)}}
$$

To normalize, we integrate the intensity over $\mathrm{x}, \mathrm{y}$, and $\mathrm{t}$ :

$$
\begin{aligned}
& \int_{-\infty}^{\infty} \int_{-\infty}^{\infty} \int_{-\infty}^{\infty} I(x, y, z, t) d x d y d t=2 \pi \int_{0}^{\infty} \frac{w_{0}^{2}}{w^{2}(z)} e^{-\frac{2 r^{2}}{w^{2}(z)}} r d r \int_{-\infty}^{\infty} \frac{2\left|A_{0}\right|^{2}}{\pi w_{0}^{2}} e^{-\frac{t^{2}}{\tau^{2}}} d t \\
& E_{0}=\frac{4\left|A_{0}\right|^{2}}{w^{2}(z)} \frac{w^{2}(z)}{4} \int_{-\infty}^{\infty} e^{-\frac{t^{2}}{\tau^{2}}} d t=\left|A_{0}\right|^{2} \sqrt{\pi} \tau
\end{aligned}
$$

To obtain the two-photon excitation signal, we square the intensity and integrate: 


$$
\begin{aligned}
& \operatorname{TPE}(z)=\int_{-\infty}^{\infty} \int_{-\infty}^{\infty} \int_{-\infty}^{\infty} I^{2}(x, y, z, t) d x d y d t=2 \pi \int_{0}^{\infty} \frac{w_{0}^{4}}{w^{4}(z)} e^{-\frac{4 r^{2}}{w^{2}(z)}} r d r \int_{-\infty}^{\infty} \frac{4\left|A_{0}\right|^{4}}{\pi^{2} w_{0}^{4}} e^{-\frac{2 t^{2}}{\tau^{2}}} d t \\
& =\frac{8\left|A_{0}\right|^{4}}{\pi w^{4}(z)} \frac{w^{2}(z)}{8} \int_{-\infty}^{\infty} e^{-\frac{2 t^{2}}{\tau^{2}}} d t=\frac{\left|A_{0}\right|^{4}}{\pi w^{2}(z)} \sqrt{\frac{\pi}{2}} \tau=\frac{E_{0}^{2}}{\pi^{2} w^{2}(z)} \sqrt{\frac{\pi}{2}} \frac{1}{\tau} \\
& =\frac{1}{w_{0}^{2}\left(1+\left(\frac{z}{z_{R}}\right)^{2}\right)} \frac{E_{0}^{2}}{\pi^{2}} \sqrt{\frac{\pi}{2}} \frac{1}{\tau}=\frac{E_{0}^{2}}{\pi^{2}} \sqrt{\frac{\pi}{2}} \frac{1}{\tau} \frac{1}{w_{0}^{2}\left(1+\left(\frac{2 z}{k w_{0}^{2}}\right)^{2}\right)}
\end{aligned}
$$

For a sample of thickness $d$, the total two-photon excitation signal per pulse is:

$$
\begin{aligned}
& \int_{-d / 2}^{d / 2} \operatorname{TPE}(z) d z=\frac{E_{0}^{2}}{\pi^{2}} \sqrt{\frac{\pi}{2}} \frac{1}{\tau} \frac{1}{w_{0}^{2}} \int_{-d / 2}^{d / 2} d z \frac{1}{\left(1+\left(\frac{2 z}{k w_{0}^{2}}\right)^{2}\right)} \\
& =\frac{E_{0}^{2}}{\pi^{2}} \sqrt{\frac{\pi}{2}} \frac{1}{\tau} k \operatorname{Arctan}\left(\frac{d}{k w_{0}^{2}}\right)
\end{aligned}
$$

Now assume that there is a surface of thickness 1 located in front of the area of interest (of thickness $d$ ) that also fluoresces. The total fluorescence due to this background region is:

$$
\begin{aligned}
\int_{-d / 2-l}^{-d / 2} \int_{0}^{2 \pi} \int_{0}^{\infty} S^{2}(\rho, z) \rho d \rho d \theta d z & =\left.\frac{\pi}{4} w_{0}^{2} z_{R} \operatorname{ArcTan}\left[\frac{z}{z_{R}}\right]\right|_{-d / 2-l} ^{-d / 2} \\
& =\frac{\pi}{4} w_{0}^{2} z_{R}\left(\operatorname{ArcTan}\left[\frac{-d}{2 z_{R}}\right]-\operatorname{ArcTan}\left[\frac{-d / 2-l}{z_{R}}\right]\right) \\
& =\frac{n_{0} \pi^{2}}{4 \lambda} w_{0}^{4}\left(\operatorname{ArcTan}\left[\frac{d / 2+l}{z_{R}}\right]-\operatorname{ArcTan}\left[\frac{d}{2 z_{R}}\right]\right)
\end{aligned}
$$

The ratio of the signal to background will therefore be:

$$
\frac{S}{B}=\frac{\frac{n_{0} \pi^{2}}{2 \lambda} w_{0}^{4} \operatorname{ArcTan}\left[\frac{d \lambda}{2 n_{0} \pi w_{0}^{2}}\right]}{\frac{n_{0} \pi^{2}}{4 \lambda} w_{0}^{4}\left(\operatorname{ArcTan}\left[\frac{(d / 2+l) \lambda}{n_{0} \pi w_{0}^{2}}\right]-\operatorname{ArcTan}\left[\frac{d \lambda}{2 n_{0} \pi w_{0}^{2}}\right]\right)}
$$

For $w_{0}=1 \mathrm{~mm}\left(z_{\mathrm{R}}=4 \mathrm{~m}\right), d=1 \mathrm{~cm}, \lambda=800 \mathrm{~nm}, n_{0}=1$, and $l=3 \mathrm{~mm}$, the SBR is 3.33. For a wavelength much smaller than the beam size, the SBR is independent of both beam size and wavelength. 


\section{D.2. Wide-field SSTF imaging}

When the dispersion is zero, the intensity at the focus in wide-field SSTF can be expressed as:

$$
I(x, y, z, t)=\left|A_{5}\right|^{2}=\left|\kappa_{x} \kappa_{y} e^{-\frac{x^{2}}{s_{x}^{2}}} e^{-\frac{y^{2}}{s_{y}^{2}}} e^{-\frac{\Omega^{2}}{4(1+\chi)}(t+\gamma x)^{2}}\right|^{2}
$$

where

$$
\begin{gathered}
\kappa_{x}=A_{0} \Omega \sqrt{\frac{i \pi f}{z_{M}}} \frac{1}{\left[-1+i\left(\frac{f-z}{z_{R}}\right)\right]^{\frac{1}{2}}}, \kappa_{y}=\frac{1}{\left(1+i \frac{2 f}{k s_{y}^{2}}\right)^{\frac{1}{2}}} \frac{1}{\left(1+i \frac{2 z}{k s_{y 1}^{2}}\right)^{\frac{1}{2}}}, \\
\gamma=\frac{k_{0} \alpha / f}{1+i(z-f) / z_{M}}, \\
\chi=\frac{i(z-f) / z_{B}}{1+i(z-f) / z_{M}}, \\
s_{x}^{2}=s_{y}^{2}=\frac{4 f^{2}}{k^{2} s^{2}}+i \frac{2(z-f)}{k}, \\
s_{y 1}^{2}=\frac{4 f^{2}}{k^{2} s^{2}}-i \frac{2 f}{k}, \\
z_{M}=\frac{2 f^{2}}{k_{0}^{2}}, z_{R}=\frac{2 f^{2} / k_{0}}{s^{2}+\alpha^{2} \Omega^{2}}, \text { and } z_{B}=\frac{2 f^{2}}{k_{0} \alpha^{2} \Omega^{2}}
\end{gathered}
$$

Substituting in these values, the intensity can be written as:

$$
I(x, y, z, t)=I_{0} e^{-\frac{x^{2}}{s_{\text {wide }}^{2}}} e^{-\frac{y^{2}}{s_{y}^{2}}} e^{-\frac{\left(t+x / v_{\text {wide }}\right)^{2}}{\tau_{\text {wide }}^{2}}}
$$

where 


$$
\begin{aligned}
& I_{0}=\left|A_{0}\right|^{2} \Omega^{2} \frac{\pi f}{z_{M}} \frac{z_{R}}{\sqrt{z_{R}^{2}+(z-f)^{2}}} \frac{f}{\sqrt{z_{M}^{2}+(z-f)^{2}}} \\
& S_{\text {wide }}=\frac{\sqrt{2} f}{k s} \sqrt{1+\frac{(z-f)^{2}}{z_{M} z_{R}}} \\
& S_{y}=\frac{\sqrt{2} f}{k s} \sqrt{1+\frac{(z-f)^{2}}{z_{M}^{2}}} \\
& \tau_{\text {wide }}=\frac{\sqrt{2}}{\Omega}\left[1+\frac{z_{M}}{z_{B}} \frac{(f-z)^{2}}{(f-z)^{2}+z_{M} z_{R}}\right]^{\frac{1}{2}} \\
& v_{\text {wide }}=\frac{f}{k \alpha}\left(1+\frac{(f-z)^{2}}{z_{M} z_{R}}\right)
\end{aligned}
$$

First we must normalize by integrating over a transverse plane to find the energy within the plane:

$$
\begin{aligned}
& \text { Energy }=\int_{-\infty}^{\infty} \int_{-\infty}^{\infty} \int_{-\infty}^{\infty} I(x, y, z, t) d x d y d t \\
& =|\kappa|^{2} \frac{\sqrt{2 \pi}}{\Omega \sqrt{\frac{z_{R}\left((f-z)^{2}+z_{M} z_{R}\right)}{z_{M}\left((f-z)^{2}+z_{R}^{2}\right)}}}\left(\int_{-\infty}^{\infty} d x e^{-\frac{x^{2}}{s_{w i d e}^{2}}}\right)\left(\frac{f}{\sqrt{z_{M}^{2}+(z-f)^{2}}} \int_{-\infty}^{\infty} d x e^{-\frac{y^{2}}{s_{y}^{2}}}\right) \\
& =\frac{\pi f}{z_{M}} \Omega^{2}\left|A_{0}\right|^{2} \frac{1}{\sqrt{1+(f-z)^{2} / z_{R}^{2}}} \frac{\sqrt{2 \pi}}{\Omega \sqrt{\frac{z_{R}\left((f-z)^{2}+z_{M} z_{R}\right)}{z_{M}\left((f-z)^{2}+z_{R}^{2}\right)}}} \cdot \ldots \\
& \ldots
\end{aligned}
$$

The pulse energy can then be written as: 


$$
\begin{aligned}
& E_{0}=\sqrt{\frac{\pi}{2}} \pi^{2}\left|A_{0}\right|^{2} s_{y} s_{x} \Omega \\
& \left|A_{0}\right|^{2}=\frac{E_{0} \sqrt{2}}{s_{y} s_{x} \Omega \pi^{5 / 2}}
\end{aligned}
$$

For the TPE at $z=f$, we get:

$$
T P E(f)=\frac{1}{16 f^{2}}\left|A_{0}\right|^{4} k^{2} \pi^{3} s^{6} \Omega^{3} \sqrt{\pi}=E^{2} \frac{k^{2} s^{2} \Omega}{8 f^{2}} \frac{\sqrt{\pi}}{\pi^{2}}
$$

To obtain the total two-photon excitation within a sample of thickness d (placed at the focus), we must integrate the squared intensity over $\mathrm{x}, \mathrm{y}$, and $\mathrm{z}$ :

$$
\begin{aligned}
& \int_{f-d / 2}^{f+d / 2} \int_{-\infty}^{\infty} \int_{-\infty}^{\infty} \int_{-\infty}^{\infty} I^{2}(x, z, t) d x d y d t d z=\int_{f-d / 2}^{f+d / 2} \int_{-\infty}^{\infty} \int_{-\infty}^{\infty} \int_{-\infty}^{\infty}\left|\kappa_{x} \kappa_{y} e^{-\frac{x^{2}}{s_{x}^{2}}} e^{-\frac{x^{2}}{s_{y}^{2}}} e^{-\frac{\Omega^{2}}{4(1+\chi)}(t+\gamma x)^{2}}\right|^{4} d x d y d t d z \\
& =\int_{f-d / 2}^{f+d / 2} d z \cdot k s^{4} \frac{\pi^{3}}{4} \Omega^{3}\left|A_{0}\right|^{4} \sqrt{\frac{\pi}{2}} \frac{z_{R}}{\sqrt{z_{R}^{2}+(z-f)^{2}}} \frac{1}{\sqrt{z_{M y}^{2}+(z-f)^{2}}} \\
& \approx \int_{f-d / 2}^{f+d / 2} d z \cdot k \frac{1}{2} \Omega \frac{E_{0}^{2}}{\pi^{2}} \sqrt{\frac{\pi}{2}} \frac{1}{\sqrt{z_{R}^{2}+(z-f)^{2}}} \frac{z_{R}}{z_{M}} \\
& =k \Omega \frac{E_{0}^{2}}{\pi^{2}} \sqrt{\frac{\pi}{2}} \frac{z_{R}}{z_{M}} \operatorname{Arcsinh}\left(\frac{d / 2}{z_{R}}\right)
\end{aligned}
$$

To be exact:

$$
\begin{aligned}
& \int_{f-d / 2}^{f+d / 2} d z \cdot k s^{4} \frac{\pi^{3}}{4} \Omega^{3}\left|A_{0}\right|^{4} \sqrt{\frac{\pi}{2}} \frac{z_{R}}{\sqrt{z_{R}^{2}+(z-f)^{2}}} \frac{1}{\sqrt{z_{M y}^{2}+(z-f)^{2}}} \\
& =k s^{4} \frac{\pi^{3}}{4} \Omega^{3}\left|A_{0}\right|^{4} \sqrt{\frac{\pi}{2}} z_{R} \int_{f-d / 2}^{f+d / 2} d z \frac{1}{\sqrt{z_{R}^{2}+(z-f)^{2}}} \frac{1}{\sqrt{z_{M y}^{2}+(z-f)^{2}}}
\end{aligned}
$$

If alpha $=0$, then

$$
\begin{aligned}
& =k s^{4} \frac{\pi^{3}}{4} \Omega^{3}\left(\frac{E_{0} \sqrt{2}}{s^{2} \Omega \pi^{5 / 2}}\right)^{2} \sqrt{\frac{\pi}{2}} z_{M} \int_{f-d / 2}^{f+d / 2} d z \frac{1}{z_{M}^{2}+(z-f)^{2}} \\
& =k \Omega \frac{E_{0}^{2}}{\pi^{2}} \sqrt{\frac{\pi}{2}} \operatorname{Arctan}\left(\frac{d / 2}{z_{M}}\right)
\end{aligned}
$$


The background can be computed as:

$$
\begin{aligned}
\int_{f-d / 2-l}^{f-d / 2} \int_{0}^{\infty} \int_{-\infty}^{\infty} I^{2}(x, z, t) d x d t d z & =-\left.z_{R} C \cdot \operatorname{Arcsinh}\left[\frac{f-z}{z_{R}}\right]\right|_{f-d / 2-l} ^{f-d / 2} \\
& =-z_{R} C \cdot\left(\operatorname{Arcsinh}\left[\frac{d / 2}{z_{R}}\right]-\operatorname{Arcsinh}\left[\frac{d / 2+l}{z_{R}}\right]\right)
\end{aligned}
$$

Therefore, the signal to background ratio can be defined as:

$$
\begin{aligned}
\frac{S}{B}= & \frac{2 z_{R} C \cdot \operatorname{Arcsinh}\left[\frac{d / 2}{z_{R}}\right]}{-z_{R} C \cdot\left(\operatorname{Arcsinh}\left[\frac{d / 2}{z_{R}}\right]-\operatorname{Arcsinh}\left[\frac{d / 2+l}{z_{R}}\right]\right)} \\
= & \frac{2}{\operatorname{Arcsinh}\left[\frac{d / 2+l}{z_{R}}\right] / \operatorname{Arcsinh}\left[\frac{d / 2}{z_{R}}\right]-1}
\end{aligned}
$$

For $z_{\mathrm{R}}=1.44 \mathrm{~mm}\left(z_{\mathrm{FWHM}}=5 \mathrm{~mm}\right), d=1 \mathrm{~cm}$, and $l=3 \mathrm{~mm}$, the SBR is 8.55 . This corresponds to an SBR improvement by a factor of 2.57 .

\section{D.3. Comparison between wide-field two-photon and SSTF imaging}

The total two-photon fluorescence generated per pulse for conventional wide-field imaging in a sample of thickness $d$ is:

$$
k \Omega \frac{E_{0}^{2}}{\pi^{2}} \sqrt{\frac{\pi}{2}} \operatorname{Arctan}\left(\frac{d / 2}{z_{M}}\right)
$$

For wide-field SSTF, the corresponding counts are:

$$
k \Omega \frac{E_{0}^{2}}{\pi^{2}} \sqrt{\frac{\pi}{2}} \frac{z_{R}}{z_{M}} \operatorname{Arcsinh}\left(\frac{d / 2}{z_{R}}\right)
$$

The ratio of SSTF to conventional counts is: 


$$
\begin{aligned}
\frac{\text { SSTF }}{\text { Conventional }}= & \frac{k \Omega \frac{E_{0}^{2}}{\pi^{2}} \sqrt{\frac{\pi}{2}} \frac{z_{R}}{z_{M}} \operatorname{Arcsinh}\left(\frac{d / 2}{z_{R}}\right)}{k \Omega \frac{E_{0}^{2}}{\pi^{2}} \sqrt{\frac{\pi}{2}} \operatorname{Arctan}\left(\frac{d / 2}{z_{M}}\right)} \\
= & \frac{\frac{z_{R}}{z_{M}} \operatorname{Arcsinh}\left(\frac{d / 2}{z_{R}}\right)}{\operatorname{Arctan}\left(\frac{d / 2}{z_{M}}\right)} \approx \frac{2 z_{R}}{d} \operatorname{Arcsinh}\left(\frac{d}{2 z_{R}}\right)
\end{aligned}
$$

For $d=1 \mathrm{~cm}$ and $z_{\mathrm{R}}=1.44 \mathrm{~mm}\left(z_{\mathrm{FWHM}}=5 \mathrm{~mm}\right)$, this ratio turns out to be $\sim 0.56$, which means that SSTF yields roughly half the number of counts per pulse.

This can be explained if we look at the TPE(z) curves for each case. For conventional wide-field imaging, the $T P E(\mathrm{z})$ can be written as:

$$
T P E_{\text {wide }}(z)=k \frac{1}{2} \Omega \frac{E_{0}^{2}}{\pi^{2}} \sqrt{\frac{\pi}{2}} \frac{1}{z_{M}\left(1+\left(\frac{z-f}{z_{M}}\right)^{2}\right)}
$$

For wide-field SSTF,

$$
T P E_{S S T F}(z)=k \frac{1}{2} \Omega \frac{E_{0}^{2}}{\pi^{2}} \sqrt{\frac{\pi}{2}} \frac{1}{z_{M}} \frac{1}{\sqrt{1+\left(\frac{z-f}{z_{R}}\right)^{2}}} \frac{1}{\sqrt{1+\left(\frac{z-f}{z_{M}}\right)^{2}}}
$$

Their coefficients are the same, so Figure D.1 is normalized to these coefficients.

Figure D.1 shows the TPE(z) curves for the conventional wide-field case (green curve) as well as the SSTF case for several values of $z_{R}$ within the range $-d / 2<z<d / 2$.

Observe that as $z_{\mathrm{R}}$ gets longer, it approaches the conventional wide-field curve. 


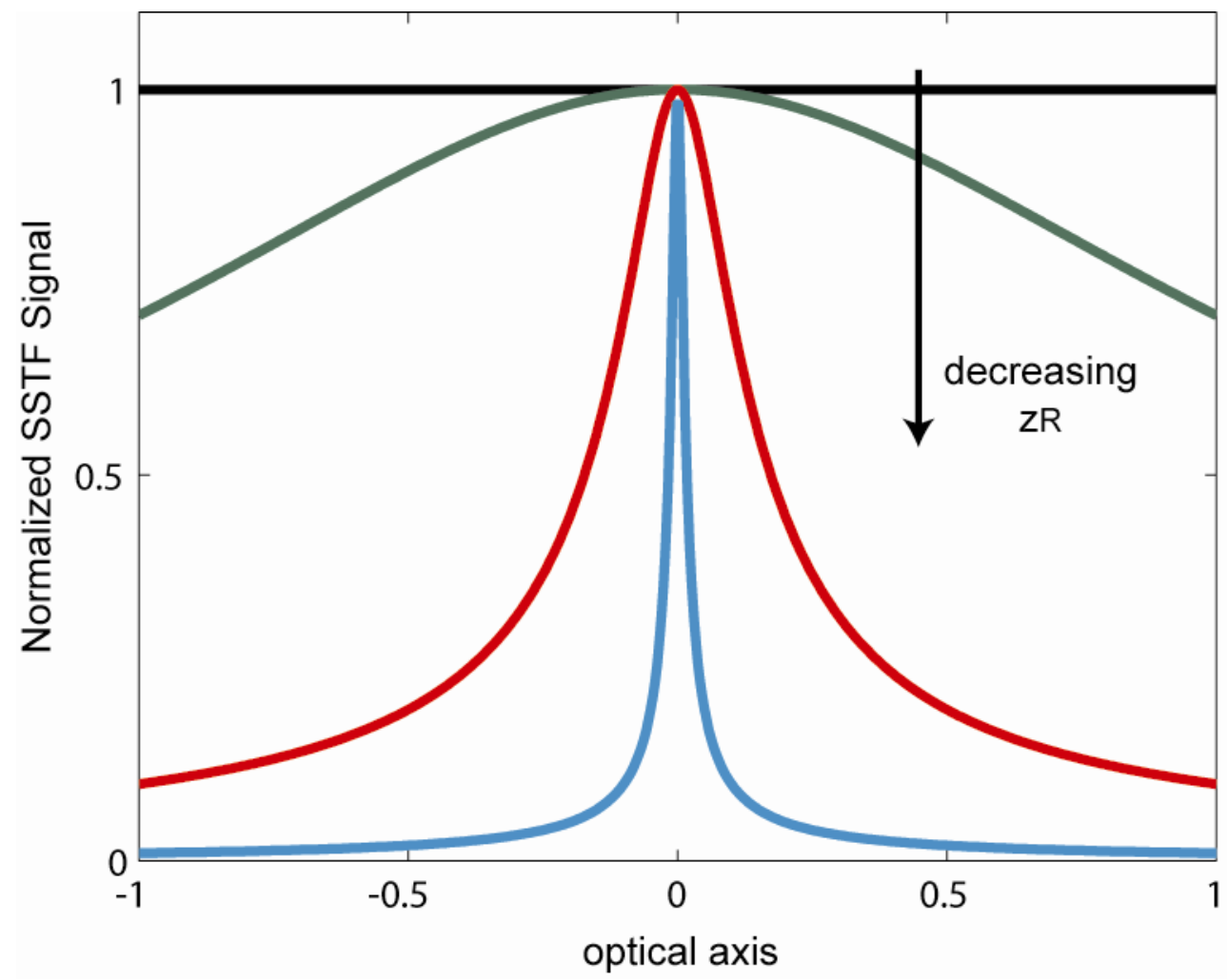

Figure D.1. $T P E_{\mathrm{SSTF}}$ curves for several values of $\mathrm{z}_{\mathrm{R}}$. The black curve represents infinite $z_{R}$. 
APPENDIX E

\section{GAUSSIAN BEAM PROPAGATION}

\section{E.1. Gaussian beam propagation starting with Maxwell's Equations ${ }^{8}$}

Maxwell's equations in free space are:

$$
\begin{gathered}
\nabla \cdot \mathbf{E}=0 \\
\nabla \cdot \mathbf{H}=0 \\
\nabla \times \mathbf{E}=-\mu_{0} \frac{\partial \mathbf{H}}{\partial t} \\
\nabla \times \mathbf{H}=\varepsilon_{0} \frac{\partial \mathbf{E}}{\partial t}
\end{gathered}
$$

Taking the curl of Eqs. (E.3) and (E.4), we get:

$$
\begin{gathered}
\nabla \times \nabla \times \mathbf{E}=\nabla \times\left(-\mu_{0} \frac{\partial \mathbf{H}}{\partial t}\right)=-\mu_{0} \frac{\partial}{\partial t}(\nabla \times \mathbf{H})=-\mu_{0} \varepsilon_{0} \frac{\partial^{2} \mathbf{E}}{\partial t^{2}} \\
\nabla \times \nabla \times \mathbf{H}=\nabla \times\left(\varepsilon_{0} \frac{\partial \mathbf{E}}{\partial t}\right)=\varepsilon_{0} \frac{\partial}{\partial t}(\nabla \times \mathbf{E})=-\mu_{0} \varepsilon_{0} \frac{\partial^{2} \mathbf{H}}{\partial t^{2}}
\end{gathered}
$$

We now make use of the vector identity:

$$
\nabla \times(\nabla \times \mathbf{E})=\nabla(\nabla \cdot \mathbf{E})-\nabla^{2} \mathbf{E}
$$

Therefore

$$
-\mu_{0} \varepsilon_{0} \frac{\partial^{2} \mathbf{E}}{\partial t^{2}}=-\nabla^{2} \mathbf{E}
$$

The wave equation can be written as

$$
\nabla^{2} \mathbf{E}-\frac{1}{c^{2}} \frac{\partial^{2} \mathbf{E}}{\partial t^{2}}=0
$$

where $c=1 /\left(\mu_{0} \varepsilon_{0}\right)$.

For a transverse electromagnetic (TEM) wave, the field components are perpendicular to the direction of propagation. We then write the electric field as:

$$
E(x, y, z)=E_{0} \cdot \psi(x, y, z) \cdot e^{-j k z}
$$

Substituting Eq. (E.10) into Eq. (E.9), we get

\footnotetext{
${ }^{8}$ The derivation in this section follows closely the formulation in Verdeyen, Laser Electronics, 1981.
} 


$$
\nabla_{t}^{2} \psi-i 2 k \frac{\partial \psi}{\partial z}+\frac{\partial^{2} \psi}{\partial z^{2}}=0
$$

We drop the second derivative with respect to $z$ because the first derivative is multiplied by $k$, a large number (SVEA). To solve, we transform to cylindrical coordinates:

$$
\frac{1}{r} \frac{\partial}{\partial r}\left(r \frac{\partial \psi}{\partial r}\right)-i 2 k \frac{\partial \psi}{\partial z}=0
$$

We guess a solution of the form:

$$
\psi=e^{-i\left(P(z)+\frac{k \cdot r^{2}}{2 q(z)}\right)}
$$

After substituting Eq. (E.13) into Eq. (E.12), we find

$$
\begin{aligned}
& q^{\prime}(z)=1 \\
& P^{\prime}(z)=-i \frac{1}{q(z)}
\end{aligned}
$$

which means $q(z)$ can be written as:

$$
q(z)=z+i z_{0}
$$

The constant term is assumed to be purely imaginary because if $q(z)$ were purely real, then the square magnitude of Eq. (E.13) would be 1 (which is uninteresting). Because we are interested in the inverse of $q(z)$, we write:

$$
\frac{1}{q(z)}=\frac{1}{z+i z_{0}}=\frac{z}{z^{2}+z_{0}^{2}}-i \frac{z_{0}}{z^{2}+z_{0}^{2}}=\frac{1}{R(z)}-i \frac{\lambda_{0}}{\pi n_{0} w^{2}(z)}
$$

where we have defined:

$$
\begin{aligned}
& w^{2}(z)=w_{0}^{2}\left[1+\left(\frac{z}{z_{0}}\right)^{2}\right] \\
& w_{0}^{2}=\frac{\lambda_{0} z_{0}}{n_{0} \pi}
\end{aligned}
$$

and

$$
R(z)=z\left[1+\left(\frac{z_{0}}{z}\right)^{2}\right]
$$

To solve for $P(z)$, we write: 


$$
\begin{aligned}
& P^{\prime}(z)=-i \frac{1}{z+i z_{0}} \\
& i P(z)=\int_{0}^{z} \frac{d z^{\prime}}{z^{\prime}+i z_{0}}=\ln \left(1-i \frac{z}{z_{0}}\right)
\end{aligned}
$$

Here, we employ the identity [1]:

$$
\ln (a+i b)=\ln \sqrt{a^{2}+b^{2}}+i \tan ^{-1}(b / a)
$$

Therefore, we can write:

$$
\begin{aligned}
& i P(z)=\ln \sqrt{1+\left(\frac{z}{z_{0}}\right)^{2}}-i \tan ^{-1}\left(\frac{z}{z_{0}}\right) \\
& e^{-i P(z)}=\frac{1}{\sqrt{1+\left(\frac{z}{z_{0}}\right)^{2}}} e^{i \tan ^{-1}\left(\frac{z}{z_{0}}\right)}=\frac{w_{0}}{w(z)} e^{i \tan ^{-1}\left(\frac{z}{z_{0}}\right)}
\end{aligned}
$$

The field can then be written as:

$$
\begin{aligned}
E(x, y, z) & =e^{-i\left(P(z)+\frac{k \cdot r^{2}}{2 q(z)}\right)} e^{-i k z} \\
& =\frac{w_{0}}{w(z)} e^{i \tan ^{-1}\left(\frac{z}{z_{0}}\right)} e^{-\frac{r^{2}}{w^{2}(z)}} e^{-i \frac{k r^{2}}{2 R(z)}} e^{-i k z}
\end{aligned}
$$

In general, we are concerned with the beam intensity:

$$
I(x, y, z)=\frac{w_{0}^{2}}{w^{2}(z)} e^{-\frac{2 r^{2}}{w^{2}(z)}}
$$

\section{E.2. Fresnel diffraction in $2 D$ with rectangular coordinates}

The purpose of this appendix is to derive an expression for the intensity $I$ of the field at the focal volume as a function of the axial position $z$. Our field at the input focal plane can be written as:

$$
A_{1}(x, y, \omega)=A_{0} e^{-\frac{x^{2}+y^{2}}{s^{2}}} e^{-\frac{\omega^{2}}{\Omega^{2}}}
$$

Using the paraxial approximation, the Fresnel diffraction equation in two dimensions [2] is: 


$$
U_{1}(x)=\frac{e^{i k f}}{i \lambda f} \int_{-\infty}^{\infty} \int_{-\infty}^{\infty} U_{0}(\xi, \eta) e^{i \frac{k}{2 f}\left[(x-\xi)^{2}+(y-\eta)^{2}\right]} d \xi d \eta
$$

We propagate the field to the objective lens with the Fresnel diffraction equation by substituting $A_{1}(x)$ from Eq. (E.24) into $U_{0}(x)$ in Eq. (E.25). The field just before the lens is therefore:

$$
\begin{aligned}
& A_{2}(x, y, \omega)=A_{0} \frac{e^{i k f}}{i \lambda f} e^{-\frac{\omega^{2}}{\Omega^{2}}} \int_{-\infty}^{\infty} \int_{-\infty}^{\infty} e^{-\frac{\xi^{2}+\eta^{2}}{s^{2}}} e^{i \frac{k}{2 f}\left[(x-\xi)^{2}+(y-\eta)^{2}\right]} d \xi d \eta \\
& =A_{0} e^{i k f} \frac{1}{\left(1+i \frac{2 f}{k s^{2}}\right)} e^{-\frac{\omega^{2}}{\Omega^{2}}} e^{-\frac{k\left(x^{2}+y^{2}\right)}{2 i f+k s^{2}}}
\end{aligned}
$$

After adding the quadratic phase due to the lens, the field becomes:

$$
A_{3}(x, y, \omega)=A_{0} e^{i k f} \frac{1}{\left(1+i \frac{2 f}{k s^{2}}\right)} e^{-\frac{k\left(x^{2}+y^{2}\right)}{2 i f+k s^{2}}} e^{-i \frac{k}{2 f}\left(x^{2}+y^{2}\right)} e^{-\frac{\omega^{2}}{\Omega^{2}}}
$$

By propagating the field a distance $z$ from the lens with the Fresnel diffraction equation (Eq. (E.25)), the field in the focal volume can be expressed as:

$$
\begin{gathered}
A_{4}(x, y, z, \omega)=A_{0} e^{i k(z+f)} \frac{1}{\left(1+i \frac{2 f}{k s^{2}}\right)\left(1+i \frac{2 z}{k s_{1}^{2}}\right)} \cdot e^{-\frac{x^{2}+y^{2}}{s_{2}^{2}}} \\
\text { where } s_{1}^{2}=\frac{4 f^{2}}{k^{2} s^{2}}-i \frac{2 f}{k} \text { and } s_{2}^{2}=\frac{4 f^{2}}{k^{2} s^{2}}+i \frac{2(z-f)}{k} .
\end{gathered}
$$

Now, we Fourier transform back into the time domain:

$$
A_{4}(x, y, z, t)=A_{0} e^{i k(z+f)} \frac{1}{\left(1+i \frac{2 f}{k s^{2}}\right)\left(1+i \frac{2 z}{k s_{1}^{2}}\right)} \cdot e^{-\frac{x^{2}+y^{2}}{s_{2}^{2}}} \sqrt{\pi} \Omega e^{-\frac{t^{2} \Omega^{2}}{4}}
$$

The intensity can therefore be written as: 


$$
\begin{aligned}
& I(x, y, z, t)=\left|A_{4}(x, y, z, t)\right|^{2}=\left|A_{0} \sqrt{\pi} \Omega e^{-\frac{t^{2} \Omega^{2}}{4}} e^{i k(z+f)} \frac{1}{\left(1+i \frac{2 f}{k s^{2}}\right)\left(1+i \frac{2 z}{k s_{1}^{2}}\right)} \cdot e^{-\frac{x^{2}+y^{2}}{s_{2}^{2}}}\right|^{2} \\
& =\left|A_{0}\right|^{2} \pi \Omega^{2} e^{-\frac{t^{2} \Omega^{2}}{2}} \frac{1}{\left(1+\left(\frac{2 f}{k s^{2}}\right)^{2}\right)} \frac{1}{\left(1+i \frac{2 z}{k s_{1}^{2}}\right)} \frac{1}{\left(1-i \frac{2 z}{k s_{1}^{* 2}}\right)} \cdot e^{-\frac{x^{2}+y^{2}}{s_{2}^{2}}} e^{-\frac{x^{2}+y^{2}}{s_{2}^{* 2}}} \\
& =\left|A_{0}\right|^{2} \pi \Omega^{2} e^{-\frac{t^{2} \Omega^{2}}{2}} \frac{1}{\left(1+\left(\frac{2 f}{k s^{2}}\right)^{2}\right)} \frac{4 f^{4}+f^{2} k^{2} s^{4}}{4 f^{4}+k^{2} s^{4}(z-f)^{2}} \cdot e^{-\left(x^{2}+y^{2}\right) \frac{8 f^{2}}{k^{2} s^{2}\left(\frac{16 f^{4}}{\left.k^{4} s^{4}+\frac{4(z-f)^{2}}{k^{2}}\right)}\right.}} \\
& =\left|A_{0}\right|^{2} \pi \Omega^{2} e^{-\frac{t^{2} \Omega^{2}}{2}} \frac{1}{k^{2} s^{4}+4 f^{2}} \frac{4 f^{4}+f^{2} k^{2} s^{4}}{\frac{4 f^{4}}{k^{2} s^{4}}+(z-f)^{2}} \cdot e^{-\left(x^{2}+y^{2}\right) \frac{8 f^{2}}{k^{2} s^{2}\left(\frac{16 f^{4}}{k^{4} s^{4}}+\frac{4(z-f)^{2}}{k^{2}}\right)}}
\end{aligned}
$$

Substitute $z_{M}=2 f^{2} /\left(k \cdot s^{2}\right)$. Then,

$$
I(x, y, z, t)=\left|A_{0}\right|^{2} \pi \Omega^{2} e^{-\frac{t^{2} \Omega^{2}}{2}} \frac{f^{2}}{z_{M}^{2}+(z-f)^{2}} \cdot e^{-\left(x^{2}+y^{2}\right) \frac{2 f^{2}}{s^{2}\left(z_{M}^{2}+(z-f)^{2}\right)}}
$$

Integration over $\mathrm{x}, \mathrm{y}$, and $\mathrm{t}$ :

$$
\begin{aligned}
& \int_{-\infty}^{\infty} \int_{-\infty}^{\infty} \int_{-\infty}^{\infty} I(x, y, z, t) d x d y d t=\left|A_{0}\right|^{2} \pi \Omega^{2} \frac{f^{2}}{z_{M}^{2}+(z-f)^{2}} \cdot \int_{0}^{\infty} 2 \pi r d r e^{-\left(r^{2}\right) \frac{2 f^{2}}{s^{2}\left(z_{M}^{2}+(z-f)^{2}\right)}} \int_{-\infty}^{\infty} d t e^{-\frac{t^{2} \Omega^{2}}{2}} \\
& E_{0}=\left|A_{0}\right|^{2} \pi \Omega^{2} \frac{f^{2}}{z_{M}^{2}+(z-f)^{2}} \cdot \frac{2 \pi s^{2}}{4 f^{2}}\left(z_{M}^{2}+(z-f)^{2}\right)\left(\frac{\sqrt{2 \pi}}{\Omega}\right) \\
& E_{0}=\left|A_{0}\right|^{2} \sqrt{\frac{\pi}{2}} \pi^{2} \Omega s^{2}
\end{aligned}
$$

which means that:

$$
\left|A_{0}\right|^{2}=\sqrt{\frac{2}{\pi}} \frac{E_{0}}{\pi^{2} \Omega s^{2}}
$$

Our final expression for the intensity then becomes: 


$$
\begin{aligned}
& I(x, y, z, t)=\sqrt{\frac{2}{\pi}} \frac{E_{0}}{\pi^{2} \Omega s^{2}} \pi \Omega^{2} e^{-\frac{t^{2} \Omega^{2}}{2}} \frac{f^{2}}{z_{M}^{2}+(z-f)^{2}} \cdot e^{-\left(x^{2}+y^{2}\right) \frac{2 f^{2}}{s^{2}\left(z_{M}^{2}+(z-f)^{2}\right)}} \\
& =\sqrt{\frac{1}{2 \pi}} \frac{E_{0}}{\pi} \Omega k e^{-\frac{t^{2} \Omega^{2}}{2}} \frac{z_{M}}{z_{M}^{2}+(z-f)^{2}} \cdot e^{-\left(x^{2}+y^{2}\right) \frac{2 f^{2}}{s^{2}\left(z_{M}^{2}+(z-f)^{2}\right)}}
\end{aligned}
$$




\section{REFERENCES}

1. J. Verdeyen, Laser Electronics (Prentice Hall, Upper Saddle River, NJ, 2000).

2. J. Goodman, Introduction to Fourier Optics (Roberts \& Company, Englewood, Colorado, 2005). 


\section{APPENDIX F}

THE EFFECT OF GROUP DELAY DISPERSION ON THE TEMPORAL WIDTH

\section{OF A GAUSSIAN PULSE}

Say we have a Gaussian pulse with a frequency-dependent phase shift $\phi(\omega)$. Expand about the central frequency $\omega_{0}[1,2]$ :

$$
\phi(\omega)=\phi\left(\omega_{0}\right)+\frac{d \phi}{d \omega}\left(\omega-\omega_{0}\right)+\frac{1}{2 !} \frac{d^{2} \phi}{d \omega^{2}}\left(\omega-\omega_{0}\right)^{2}+\frac{1}{3 !} \frac{d^{3} \phi}{d \omega^{3}}\left(\omega-\omega_{0}\right)^{3}+\ldots
$$

where

$$
\beta_{m}=\left(\frac{d^{m} \beta}{d \omega^{m}}\right)_{\omega=\omega_{0}}
$$

Let us focus on $\beta_{2}$. For a Gaussian pulse with a central frequency $\omega_{0}$, the electric field can be written as:

$$
E_{1}(t)=E_{0} e^{-\frac{t^{2}}{2 \tau_{0}^{2}}} e^{i \omega_{0} t}
$$

Fourier transforming into the frequency domain, we obtain:

$$
\begin{aligned}
E_{1}(\omega) & =\int_{-\infty}^{\infty} E_{0} e^{-\frac{t^{2}}{2 \tau_{0}^{2}}} e^{i \omega_{0} t} e^{-i \omega t} d t=\int_{-\infty}^{\infty} E_{0} e^{-\frac{t^{2}}{2 \tau_{0}^{2}}} e^{-i\left(\omega-\omega_{0}\right) t} d t \\
& =E_{0} \int_{-\infty}^{\infty} E_{0} e^{-\frac{t^{2}}{2 \tau_{0}^{2}}} e^{-i\left(\omega-\omega_{0}\right) t} e^{\gamma} e^{-\gamma} d t
\end{aligned}
$$

First, we must complete the square [3]:

$$
\frac{t^{2}}{2 \tau_{0}^{2}}+i\left(\omega-\omega_{0}\right) t+\gamma=\left(\frac{t}{\sqrt{2} \tau_{0}}+\alpha\right)^{2}=\frac{t^{2}}{2 \tau_{0}^{2}}+\frac{2 \alpha t}{\sqrt{2} \tau_{0}}+\alpha^{2}
$$

which means that

$$
\begin{aligned}
\frac{2 \alpha t}{\sqrt{2} \tau_{0}} & =i\left(\omega-\omega_{0}\right) t \\
\alpha & =\frac{i\left(\omega-\omega_{0}\right) \sqrt{2} \tau_{0}}{2}
\end{aligned}
$$

Therefore, the Fourier transform can be rewritten as: 


$$
\begin{aligned}
E_{1}(\omega) & =E_{0} \int_{-\infty}^{\infty} e^{-\left(\frac{t}{\sqrt{2} \tau_{0}}+\frac{1}{2} i\left(\omega-\omega_{0}\right) \sqrt{2} \tau_{0}\right)^{2}} e^{-\frac{\left(\omega-\omega_{0}\right)^{2} \tau_{0}^{2}}{2}} d t \\
& =E_{0} \sqrt{2 \pi} \tau_{0} e^{-\frac{\left(\omega-\omega_{0}\right)^{2} \tau_{0}^{2}}{2}}
\end{aligned}
$$

where we have made use of the integral identity:

$$
\int_{-\infty}^{\infty} e^{-q^{2}} d q=\sqrt{\pi}
$$

Now, we add the effects of dispersion in the frequency domain:

$$
E_{2}(\omega)=E_{0} \sqrt{2 \pi} \tau_{0} e^{-\frac{\left(\omega-\omega_{0}\right)^{2} \tau_{0}^{2}}{2}} e^{i \beta_{0} z} e^{i \beta_{1}\left(\omega-\omega_{0}\right) z} e^{i \frac{1}{2} \beta_{2}\left(\omega-\omega_{0}\right)^{2} z}
$$

We now ignore the zero and first order terms in Eq. (F.9). Fourier transforming back into the time domain:

$$
\begin{aligned}
E_{2}(t) & =\frac{1}{2 \pi} \int_{-\infty}^{\infty} E_{0} \sqrt{2 \pi} \tau_{0} e^{-\frac{\left(\omega-\omega_{0}\right)^{2} \tau_{0}^{2}}{2}} e^{i \frac{1}{2} \beta_{2}\left(\omega-\omega_{0}\right)^{2} z} e^{i \omega t} d \omega \\
& =\frac{1}{2 \pi} E_{0} \sqrt{2 \pi} \tau_{0} \int_{-\infty}^{\infty} e^{-\left(\omega-\omega_{0}\right)^{2}\left(\frac{\tau_{0}^{2}}{2}-i \frac{1}{2} \beta_{2} z\right)} e^{i \omega t} d \omega
\end{aligned}
$$

Change of variables makes:

$$
\begin{aligned}
& x=\omega-\omega_{0} ; \\
& E_{2}(t)=\frac{1}{2 \pi} E_{0} \sqrt{2 \pi} \tau_{0} e^{i \omega_{0} t} \int_{-\infty}^{\infty} e^{-x^{2}\left(\frac{\tau_{0}^{2}}{2}-i \frac{1}{2} \beta_{2} z\right)} e^{i x t} d x
\end{aligned}
$$

Again, we must complete the square:

$$
\begin{aligned}
\mathrm{x}^{2}\left(\frac{\tau_{0}^{2}}{2}-i \frac{1}{2} \beta_{2} z\right)+i t x+\Gamma & =\left(x \sqrt{\frac{\tau_{0}^{2}}{2}-i \frac{1}{2} \beta_{2} z}+\mathrm{A}\right)^{2} \\
2 \mathrm{~A} \sqrt{\frac{\tau_{0}^{2}}{2}-i \frac{1}{2} \beta_{2} z} & =i t \\
\mathrm{~A} & =\frac{i t}{2 \sqrt{\frac{\tau_{0}^{2}}{2}-i \frac{1}{2} \beta_{2} z}} \\
\Gamma & =\frac{-t^{2}}{2\left(\tau_{0}^{2}-i \beta_{2} z\right)}
\end{aligned}
$$


This makes the integral become:

$$
E_{2}(t)=\frac{1}{2 \pi} E_{0} \sqrt{2 \pi} \tau_{0} e^{i \omega_{0} t} e^{\frac{-t^{2}}{2\left(\tau_{0}^{2}-i \beta_{2} z\right)}} \int_{-\infty}^{\infty} e^{-\left(x \sqrt{\frac{\tau_{0}^{2}}{2}-i \frac{1}{2} \beta_{2} z}+\frac{i t}{2 \sqrt{\frac{\tau_{0}^{2}}{2}-i \frac{1}{2} \beta_{2} z}}\right)^{2}} d x
$$

Make a substitution to complete the integration:

$$
\begin{aligned}
y & =x \sqrt{\frac{\tau_{0}^{2}}{2}-i \frac{1}{2} \beta_{2} z}+\frac{i t}{2 \sqrt{\frac{\tau_{0}^{2}}{2}-i \frac{1}{2} \beta_{2} z}} \\
d y & =d x \sqrt{\frac{\tau_{0}^{2}}{2}-i \frac{1}{2} \beta_{2} z} \\
E_{2}(t) & =\frac{1}{2 \pi} E_{0} \sqrt{2 \pi} \tau_{0} e^{i \omega_{0} t} e^{\frac{-t^{2}}{2\left(\tau_{0}^{2}-i \beta_{2} z\right)}} \frac{1}{\sqrt{\frac{\tau_{0}^{2}}{2}-i \frac{1}{2} \beta_{2} z}} \int_{-\infty}^{\infty} e^{-y^{2}} d y
\end{aligned}
$$

Therefore, the field can be written as:

$$
\begin{aligned}
E_{2}(t) & =\frac{1}{2 \pi} E_{0} 2 \pi \tau_{0} e^{i \omega_{0} t} e^{\frac{-t^{2}}{2\left(\tau_{0}^{2}-i \beta_{2} z\right)}} \frac{1}{\sqrt{\frac{\tau_{0}^{2}}{2}-i \frac{1}{2} \beta_{2} z}} \\
& =E_{0} e^{i \omega_{0} t} e^{\frac{-t^{2}}{2\left(\tau_{0}^{2}-i \beta_{2} z\right)}} \frac{\tau_{0}}{\sqrt{\tau_{0}^{2}-i \beta_{2} z}}
\end{aligned}
$$

Therefore, the Gaussian pulse keeps its shape, but its pulse width broadens with increasing z. This can be seen more clearly in the expression for the intensity:

$$
\begin{aligned}
& I_{2}(t)=\left|E_{2}(t)\right|^{2}=E_{0}^{2} e^{\frac{-t^{2}}{\tau^{2}+\left(\frac{\beta_{2} z}{\tau_{0}}\right)^{2}}} \frac{\tau_{0}^{2}}{\sqrt{\tau_{0}^{4}+\left(\beta_{2} z\right)^{2}}} \\
& \tau(z)=\tau_{0} \cdot\left[1+\left(\frac{\beta_{2} z}{\tau_{0}^{2}}\right)^{2}\right]^{\frac{1}{2}}=\tau_{0} \cdot\left[1+\left(\frac{z}{L_{D}}\right)^{2}\right]^{\frac{1}{2}}
\end{aligned}
$$

where the dispersion length, $L_{\mathrm{D}}$, is defined as:

$$
L_{D}=\frac{\tau_{0}^{2}}{\left|\beta_{2}\right|}
$$


Note: If using the FWHM pulse width, then substitute:

$$
\tau_{\text {FWHM }}=2 \sqrt{\ln 2} \cdot \tau_{0} \approx 1.665 \cdot \tau_{0}
$$




\section{REFERENCES}

1. P M W French. "The generation of ultrashort laser pulses." Rep. Prog. Phys. 58 (1995) 169-267.

2. G P Agrawal. Nonlinear Fiber Optics. Third Edition. New York: Academic Press, 2001.

3. B Kusse and E Westwig. Mathematical Physics. New York: John Wiley and Sons, Inc., 1998. 